Linköping University Medical Dissertations No. 1418

\title{
Methods to Reduce Liver Ischemia/Reperfusion Injury
}

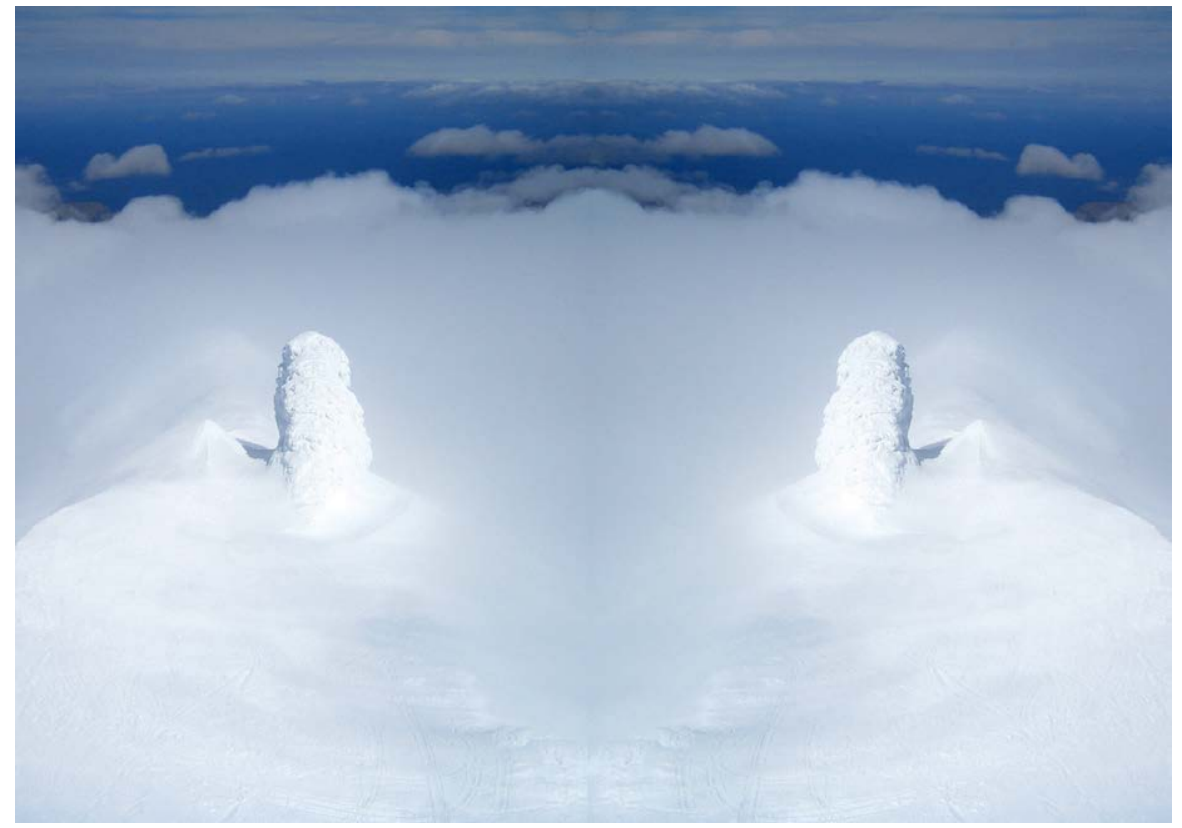

Bergpór Björnsson

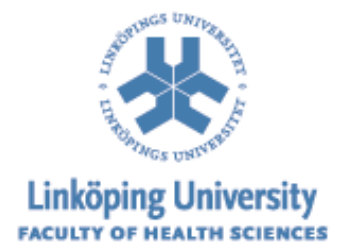

Division of Surgery

Department of Clinical and Experimental Medicine

Faculty of Health Sciences

Linköping University

Linköping 2014 
Copyright (C) Bergthor Björnsson, 2014

bergthor.bjornsson@lio.se

Published articles have been reprinted with the permission of the copyright holder.

Printed in Sweden by LiU-Tryck, Linköping, Sweden, 2014

ISSN 0345-0082

ISBN 978-91-7519-245-1 


\title{
Supervisor
}

Per Sandström, MD, PhD, Associate Professor

Division of Surgery

Department of Clinical and Experimental Medicine

Faculty of Health Sciences, Linköping University

\author{
Assistant Supervisors \\ Per Gullstrand, MD, PhD, Associate Professor \\ Division of Surgery \\ Department of Clinical and Experimental Medicine \\ Faculty of Health Sciences, Linköping University
}

Tommy Sundqvist, PhD, Professor

Division of Medical Microbiology

Department of Clinical and Experimental Medicine

Faculty of Health Sciences, Linköping University

Anders Winbladh, MD, PhD

\section{Opponent}

Styrbjörn Friman, MD, PhD, Professor

Department of Surgery

Institute of Clinical Sciences

The Sahlgrenska Academy, University of Gothenburg

\section{Host}

Per Sandström, MD, PhD, Associate Professor

Division of Surgery

Department of Clinical and Experimental Medicine

Faculty of Health Sciences, Linköping University 


\section{Committee board}

Bengt Isaksson, $\mathrm{MD}, \mathrm{PhD}$, Associate Professor

Division of Surgery

Department of Clinical Science, Intervention and Technology

Karolinska Institutet

Lennart Nilsson, MD, PhD, Associate Professor

Division of Cardiovascular Medicine

Department of Medical and Health Sciences

Faculty of Health Sciences, Linköping University

Anders Kald, MD, PhD, Associate Professor

Division of Surgery

Department of Clinical and Experimental Medicine

Faculty of Health Sciences, Linköping University 
To:

Gunna, Hilda and Viktor

"Education is what survives when what has been learned has been forgotten"

Burrhus Frederic Skinner (1904-1990)

Cover photograph:

Snæfellsjökull, Iceland

Revised, and published with the permission of H.J. Larusson 



\section{Contents}

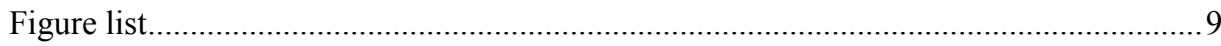

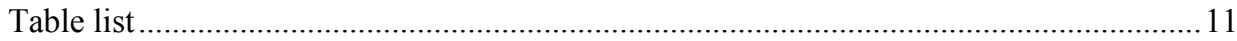

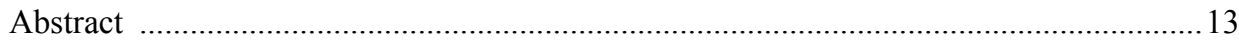

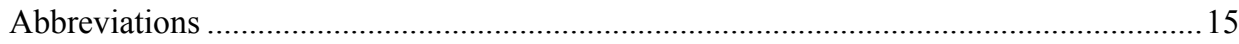

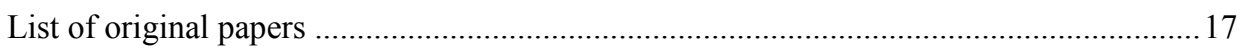

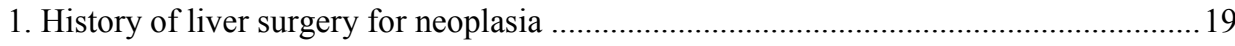

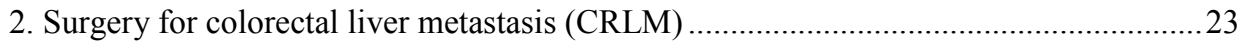

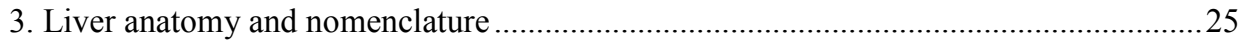

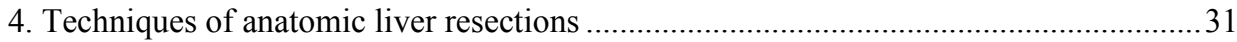

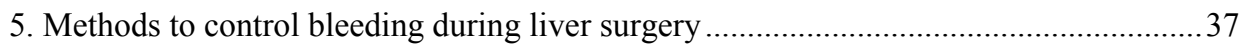

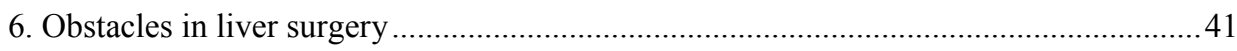

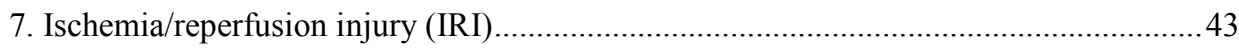

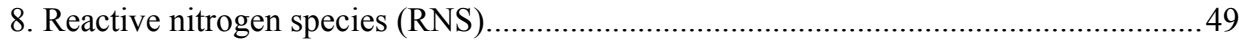

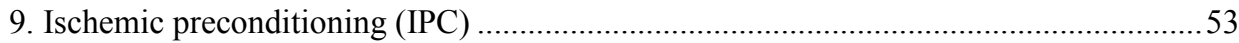

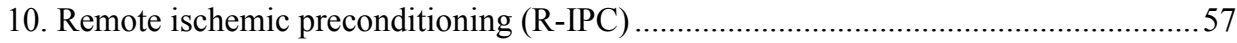

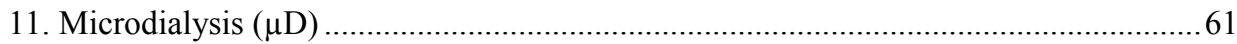

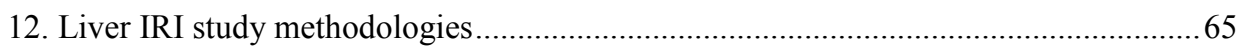

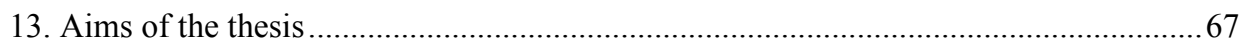

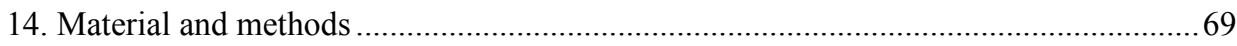

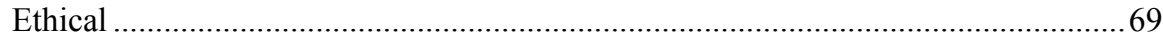

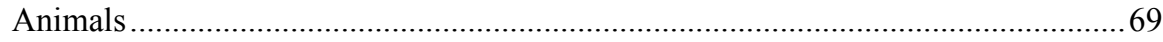

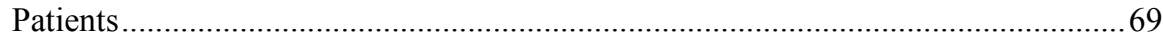

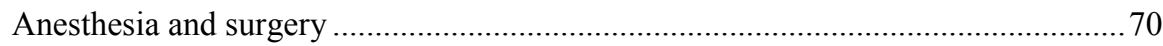

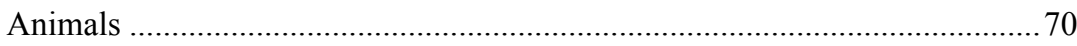

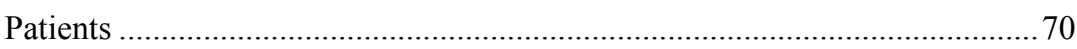

IPC, R-IPC and liver ischemia (studies I, III and IV)......................................... 70

Administration of nitrite (study IV) ....................................................................

Local metabolism (microdialysis) …................................................................. 71

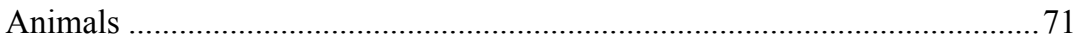

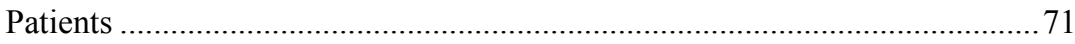

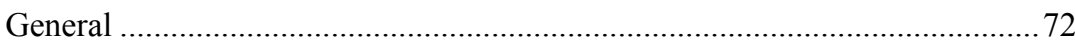




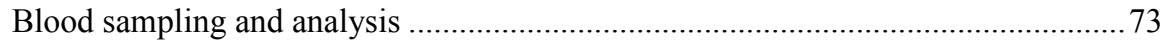

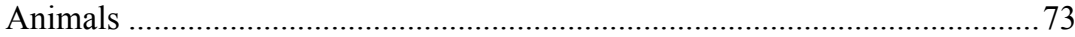

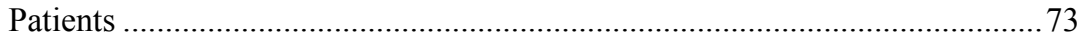

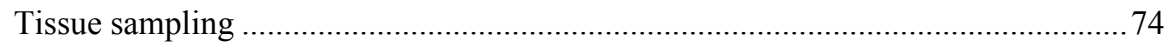

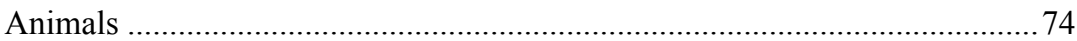

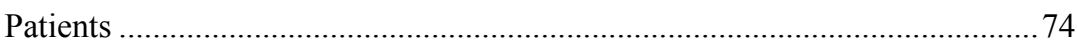

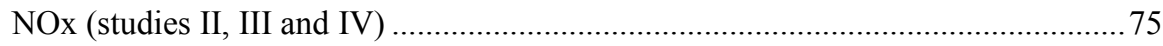

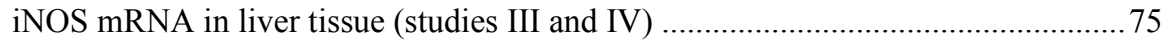

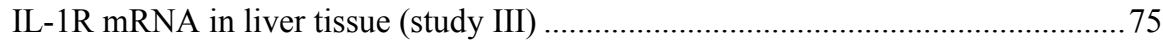

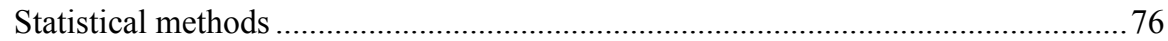

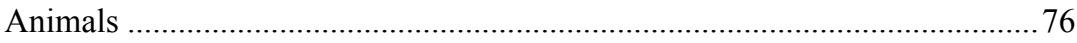

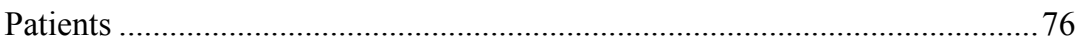

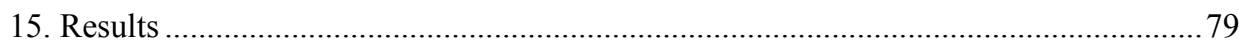

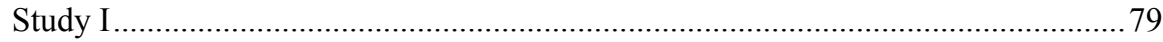

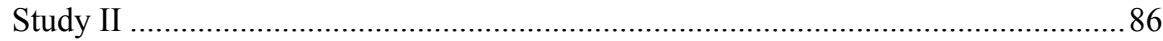

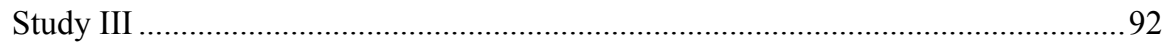

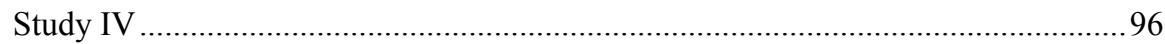

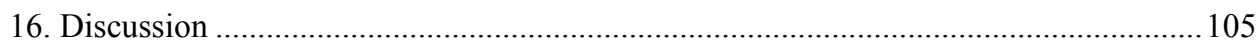

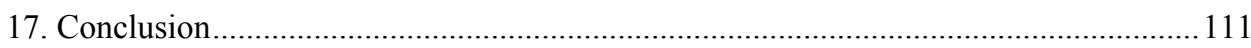

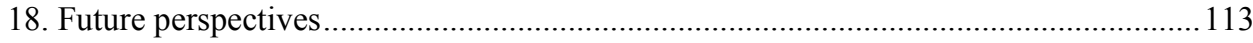

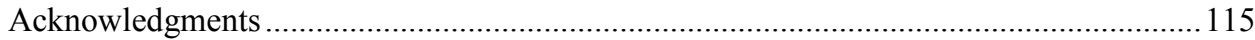

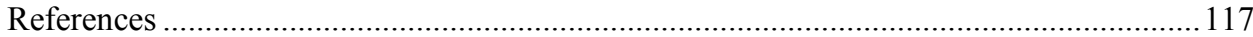




\section{Figure list}

Figure 1. Number of liver resections (JJBxx) in Sweden 1998-2012 ..............................23

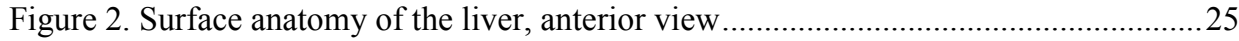

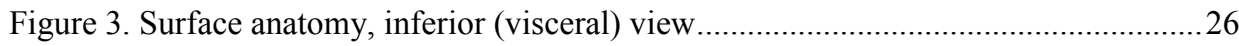

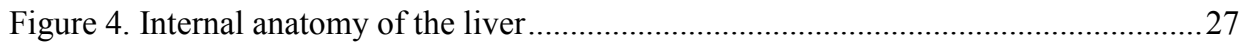

Figure 5. Cavitron Ultrasonic Surgical Aspirator (CUSA) .............................................. 31

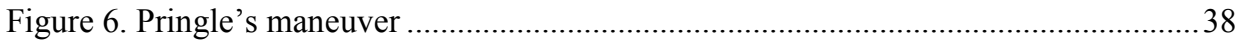

Figure 7. The major cells and mediators involved in liver IRI .......................................4

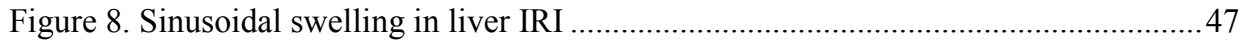

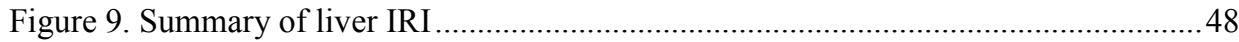

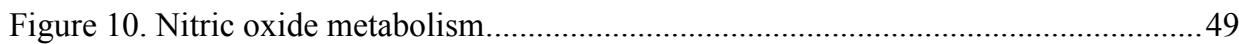

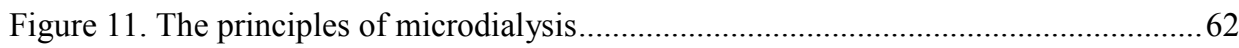

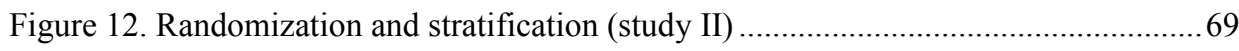

Figure 13. Microdialysis in a clinical setting (study II) ................................................ 71

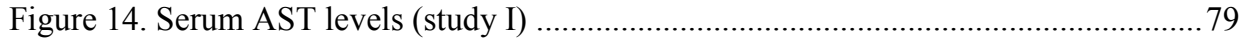

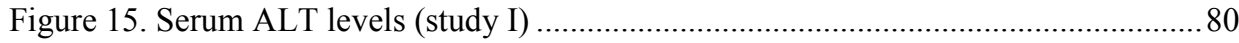

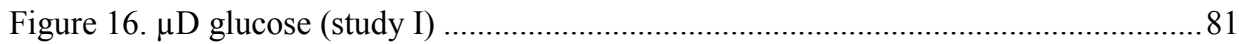

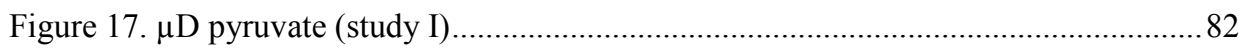

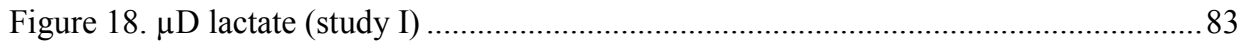

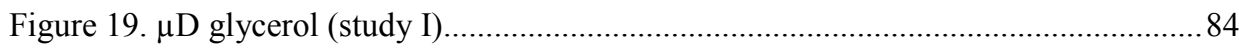

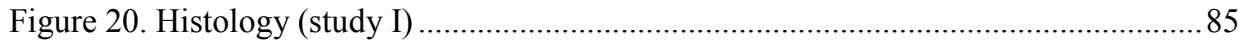

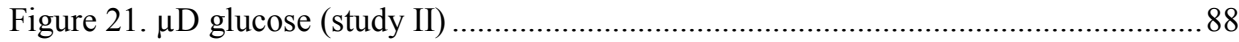

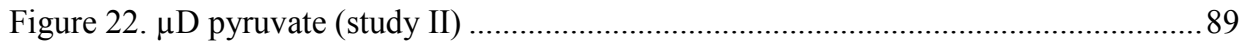

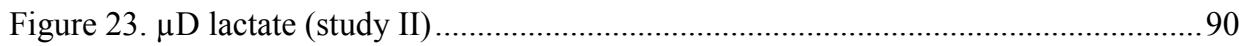

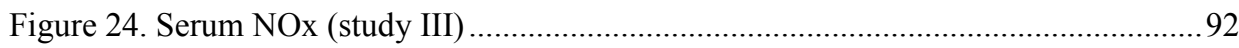

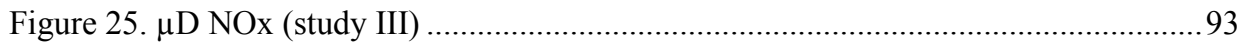

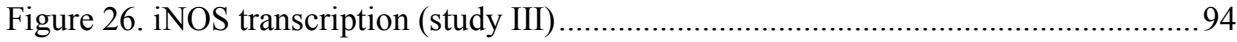

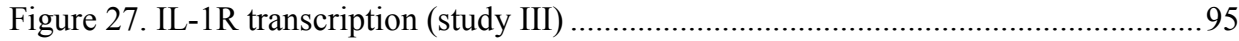

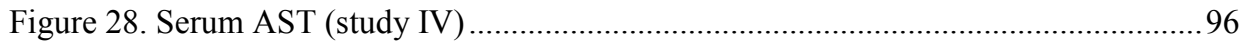

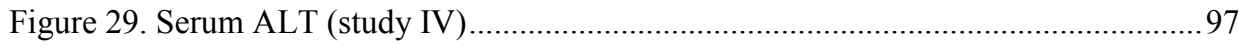

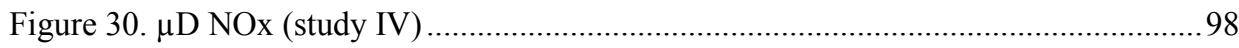

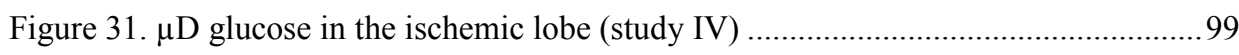

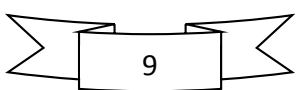




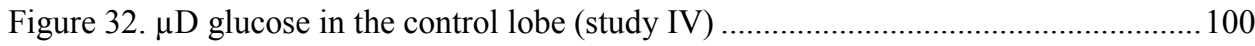

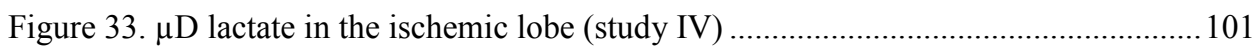

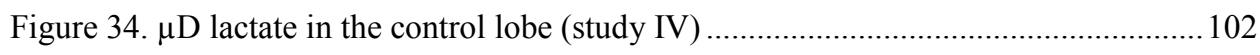

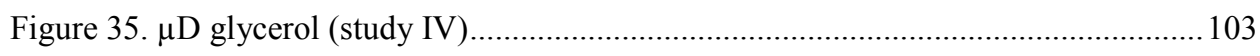

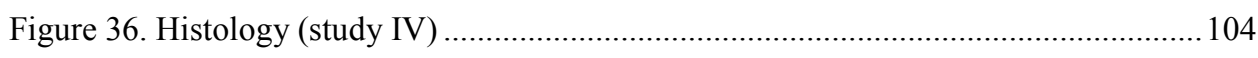

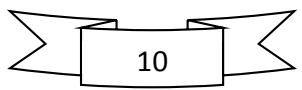




\section{Table list}

Table 1. Comparison of the classifications systems used to describe the internal anatomy of

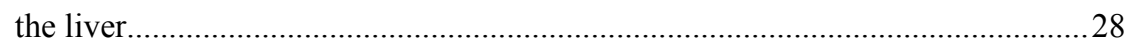

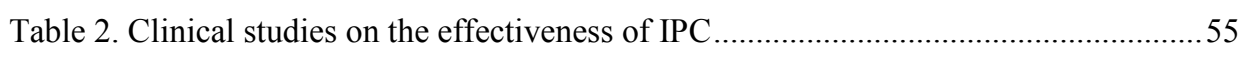

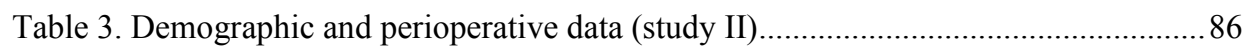

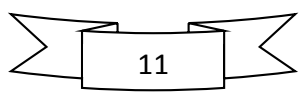


$\sum[12]$ 


\section{Abstract}

Introduction: During the last two decades, liver surgery has expanded enormously, partly due to improved surgical equipment and techniques as well as new and more powerful chemotherapy agents. As the liver is a very well-vascularized organ, there is an inherent risk of bleeding during liver resection. One of the most popular methods employed to reduce this risk is to close the vascular inflow to the liver using the Pringle's maneuver (PM). However, this procedure has been recognized to cause ischemia/reperfusion injury (IRI) to the future liver remnant (FLR). In cases of extensive resection where the FLR is small and in cases when the liver suffers from chronic diseases, such as cirrhosis, IRI can greatly increase the risk of post-operative liver failure (POLF). Ischemic preconditioning (IPC) and, more recently, remote ischemic preconditioning (R-IPC) are methods that have been employed to reduce IRI.

Aim: 1) To compare the effects of IPC and R-IPC in a rat model; 2) to investigate the clinical effect of IPC during modern liver surgery; 3 ) to investigate the role of the nitric oxide (NO) system in IRI, IPC and R-IPC; and 4) to explore the possible protective effects of nitrite administration before IRI.

Methods: A rat model of segmental ischemia followed by 4 hours of reperfusion including microdialysis $(\mu \mathrm{D})$ was developed from earlier models. The effects of IPC and R-IPC were compared using transaminases and histology as well as continuous $\mu \mathrm{D}$ sampling for glucose, pyruvate, lactate and glycerol. The role of the NO system was examined by serum and $\mu \mathrm{D}$ measurements of NOx as well as tissue measurements of iNOS mRNA and IL-1R mRNA. In study II, patients were randomized to IPC or no IPC prior to liver resection, where intermittent PM was used to decrease bleeding.

Results: IPC was more effective in protecting the liver against IRI than R-IPC, as indicated by the levels of transaminases. Lower lactate levels were detected in patients treated with IPC before major liver resections than in controls. IPC reduced iNOS mRNA transcription during reperfusion; this result may be related to the early but not sustained increases in IL-1R transcription observed in the IPC group. Nitrite administered before ischemia reduced AST and ALT levels in the level after 4 hours of reperfusion; in addition, necrosis and glycerol release from the ischemic liver were reduced as well.

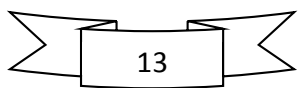


Conclusion: IPC is more effective than R-IPC in animal models; however, this effect is unlikely to be of clinical importance. NOx decreases in the ischemic liver and the administration of nitrite before ischemia reduces IRI in rats. This may have clinical implications in the future.

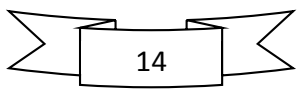




\section{Abbreviations}

ALPPS = Associating liver partition and portal vein ligation for staged hepatectomy

$\mathrm{ALT}=$ Alanine aminotransferase

ANOVA $=$ Analysis of variance

$\mathrm{AST}=$ Aspartate aminotransferase

$\mathrm{ATP}=$ Adenosine triphosphate

cGMP $=$ Cyclic guanosine monophosphate

$\mathrm{cPM}=$ Continuous Pringle's maneuver

C-PTIO = 2-(4.carboxyphenyl)-4,4,5,5-tetramethylimiadozoline-1-ocyl-3-oxide potassium salt

CRLM $=$ Colorectal liver metastasis

CUSA $=$ Cavitron Ultrasonic Surgical Aspirator

$\mathrm{CVP}=$ Central venous pressure

$\mathrm{DAN}=2,3$ diaminonapthalene

ELISA $=$ Enzyme-linked immunosorbent assay

eNOS $=$ Endothelial nitric oxide synthase

$\mathrm{FLR}=$ Future liver remnant

GAPDH = Glyceraldehyde-3-phosphate-dehydrogenase

$\mathrm{HCC}=$ Hepatocellular carcinoma

HO-1 = Heme oxygenase 1

ICG-15 = 15 minute indocyanine green clearance test

ICAM $=$ Intercellular adhesion molecule

$\mathrm{ICG}=$ Indocyanine green

IFN- $\gamma=$ Interferon $\gamma$

$\mathrm{IL}=$ Interleukin

IL-1R $=$ Interleukin 1 receptor

iNOS = Inducible nitric oxide synthase

$\mathrm{INR}=$ International normalized ratio

$\mathrm{IPC}=$ Ischemic preconditioning

iPM $=$ intermittent Pringle's maneuver

$\mathrm{IR}=$ Ischemia/reperfusion

IRI = Ischemia/reperfusion Injury 
$\mathrm{L}-\mathrm{NAME}=\mathrm{N}^{\mathrm{G}}$ nitro-L-arginine methyl ester

MAC-1 = Macrophage 1 antigen

$\mu \mathrm{D}=$ Microdialysis

$\mathrm{NADPH}=$ Nicotinamide adenine dinucleotide phosphate

$\mathrm{NF}-\kappa \mathrm{B}=$ Nuclear factor $\kappa \mathrm{B}$

nNOS $=$ Neuronal nitric oxide synthase

$\mathrm{NO}=$ Nitric oxide

$\mathrm{NOx}=$ The sum of nitrite and nitrate

ODQ $=1 \mathrm{H}-(1,2,3)$ oxadiazole(4,3-a)quinoxalin-1-one

$\mathrm{PM}=$ Pringle's maneuver

$\mathrm{PMN}=$ Polymorphonuclear neutrophil

POLF $=$ Post-operative liver failure

PVE $=$ Portal vein embolization

$\mathrm{R}-\mathrm{IPC}=$ Remote ischemic preconditioning

RNS $=$ Reactive nitrogen species

ROS $=$ Reactive oxygen species

$\mathrm{SD}=$ Standard deviation

$\mathrm{SEM}=$ Standard error of the mean

$\mathrm{sGC}=$ Soluble guanylyl cyclase

SHVE $=$ Selective hepatic vascular exclusion

THVE $=$ Total hepatic vascular exclusion

TLR4 $=$ Toll-like receptor 4

TNF- $\alpha=$ Tumor necrosis factor $\alpha$

$\mathrm{WBC}=$ White blood cells 


\section{List of original papers}

The thesis is based on the following original articles, which are referenced in-text by the respective roman numerals:

I. B. Björnsson, A. Winbladh, L. Bojmar, L. Trulsson, H. Olsson, T. Sundqvist, P. Gullstrand, P. Sandström.

Remote or Conventional Ischemic Preconditioning - Local Liver

Metabolism in Rats Studied with Microdialysis

Journal of surgical research 2012;176(1):55-62

II. A. Winbladh, B. Björnsson, L. Trulsson, K. Offenbartl, P. Gullstrand, P. Sandström

Ischemic preconditioning prior to intermittent Pringle maneuver in liver resections

Journal of Hepatobiliary Pancreatic Sci 2012;19(2):159-170

III. B. Björnsson, A. Winbladh, L. Bojmar, T. Sundqvist, P. Gullstrand, P. Sandström

Conventional, but not remote ischemic preconditioning, reduces iNOS transcription in liver ischemia/reperfusion

World J Gastroenterol 2014; 20(28):9506-9512

IV. B. Björnsson, L. Bojmar, H. Olsson, T. Sundqvist, P. Sandström

Nitrite, a novel method to decrease Ischemia/Reperfusion Injury in the rat liver

Provisionally accepted for publication in World J Gastroenterol 
$\sum[18]$ 


\section{History of liver surgery for neoplasia}

Homer wrote in The Iliad:

"Achilles stabbed with his sword at the liver, the liver was torn from its place, and from it the dark blood drenched the fold of his tunic and Troy's eyes were shrouded in darkness, and the light went out" (1).

This description from approximately 750 B.C. may be one of the earliest descriptions of liver trauma and certainly addresses one of the main risks of liver surgery, bleeding. The words attributed to Sir William Osler, "if there wasn't bleeding everybody would do surgery", are particularly applicable to liver surgery.

Before the introduction of the endotracheal tube in 1878 by William Macewen, elective abdominal surgery was almost unknown (2). The liver was only operated on in cases of trauma, and those operations were mostly or exclusively performed through wounds already present from the trauma. The first documented successful liver operation for trauma was performed by Wilhelm Fabry (Fabricius Hildanus), known as "the father of German surgery", in the $17^{\text {th }}$ century and consisted of removal of a portion of the liver present in a trauma wound (3).

Despite Lord Thurlow's statement at the parliamentary debate on the establishment of Royal College of Surgeons in 1811, "There is no more science in surgery than in butchering", some documentation can be found concerning the early days (until the mid-20 $0^{\text {th }}$ century) of planned liver surgery. In 1873, the British surgeon Sir John Eric Erichsen announced: "The abdomen, the chest, and the brain will be forever shut from the intrusion of the wise and humane surgeon." (4). Despite Dr. Erichsen's pessimism, the first documented planned hepatectomy was performed in 1886 by Dr. A Lius in Italy. The result was a deadly complication that later served as the cornerstone of liver surgery development: the patient bled to death 6 hours after surgery $(5,6)$. However, the die was rolled, and in 1888 , the German surgeon Carl Johann August Langenbuch, who performed the first cholecystectomy in 1882, performed the first successful liver resection for a tumor. A portion of the left liver lobe was resected after first ligating the vascular pedicles (7). Although the patient survived, a reoperation for bleeding was required; unfortunately, the pathological examination revealed no malignancy. Concurrently, in the USA, liver surgery was also evolving, and in 1890, Louis McLane Tiffany, a professor of surgery at the University of Maryland in Baltimore,

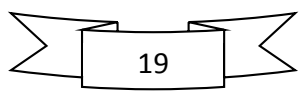


performed the first liver resection for a tumor at Johns Hopkins Hospital (8). This achievement was closely followed by the first reported liver resection for a malignant tumor by another German surgeon, Lucke, in 1891 (9).

Sir James Cantlie published a significant contribution to the understanding of functional liver anatomy in 1897 by describing the true line separating the right and left liver lobes; however, this discovery was only first applied to clinical use a few decades later (10). In 1899, when Keen reported the first anatomical left lateral segmentectomy (segments II and III), he also updated his previous two reviews and noted that a total of 76 liver resections had been reported with a mortality rate of only $14.9 \%$ (11). Whether this represents publication bias or true progress in the field remains unclear, although Dr. Keen addresses over 20 cases that he excludes from the analysis as not being liver resections.

In 1903, Dr. W Anschutz described the technique called the "finger fracture", which was later made popular by Lin $(12,13)$. Seven years earlier (1896), a technique based on the same principles, wherein sutures were passed through the liver tissue to create pressure before dividing the parenchyma, was described by Kousnetzoff and Pensky (14). The finger fracture technique was later further improved by Lin through the use of clamps along with the fingers (15).

In 1908, trauma surgeons made a substantial contribution addressing bleeding. While working at the Glasgow Royal Infirmary in 1908, Dr. James Hogarth Pringle described a novel method to minimize bleeding resulting from liver trauma; he developed his method using an animal model and tested it on two patients (16). Although neither of the patients survived, Pringle's maneuver (PM), which involves temporary closure of the hepatoduodenal ligament along with the finger fracture, is likely the oldest technique in liver surgery that remains in use. This method of bleeding control was previously proposed by Clementi in 1890 (17).

The first major hepatectomy was performed by Wendel in 1911 and in 1920 . While the patient was still alive, Wendel published the case of hepatocellular carcinoma (HCC) (18). In the Western world, liver surgery for malignant diseases is dominated by the resection of metastases, primarily from colorectal carcinoma $(19,20)$. The first reported hepatic metastasectomy for colorectal carcinoma was performed at the Lahey Clinic in Burlington, Massachusetts in 1940 by Dr. Richard B. Cattell (21). The first well-defined formal right hepatectomy was performed at the Beaujon Hospital in Paris by JL Lortat-Jacob in 1952 (2224). This surgery marked the beginning of French involvement in liver surgery, which

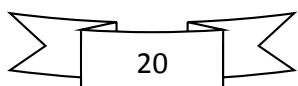


increased during the latter half of the $20^{\text {th }}$ century both in the fields of anatomy and nomenclature as well as in the introduction of two-stage liver resections (25-27). A year later, in 1953, Julian K. Quattlebaum, working in Savannah, Georgia, USA, reported his series of three major hepatectomies, placing the USA on the map of anatomical liver surgery (28).

The French surgeon and anatomist Claude Couinaud published the book Le foie; etudes anatomiques et chirurgicales (The liver: Anatomical studies and surgical studies) in 1957. This 530-page book describes in detail the segmental anatomy of the liver and is the foundation of the nomenclature used in Europe and Japan within liver surgery (26).

A review of the history of liver surgery cannot exclude liver transplantation. The first liver transplantation was performed in Denver, Colorado, USA in 1963 by a team led by Dr. Thomas Starzl (29). The first one-year survival was achieved in 1968 (for an operation that occurred in 1967). With the introduction of cyclosporin, liver transplantation became a viable clinical option in the 1980s. This step opened the door for more complex resections of the liver, ranging from in situ in vivo (with vascular exclusion of the organ and hypothermia by perfusion) as described by Fortner (Memorial Sloan-Kettering) in 1974 to ex situ ex vivo (where the liver is temporary removed from the body and operated on a back table) as performed by Pichlmayr (Hannover) in 1990 (30, 31).

One of the more significant advances in the diagnosis of liver neoplasia was the introduction of the ultrasound technique in the 1970s (32). Ultrasound spread fast in clinical practice, and its use during operations was introduced in Japan in 1983 and in France in 1984 $(33,34)$.

In his work published in 1987, Sir James Cantlie's stated:

"I believe that if, in the hands of future observers, the statements I have made receive closer investigation, the surgery of the liver will be advanced a step" (10).

His anatomical observations involving the hypertrophy of one liver lobe, the atrophy of the other, and the separate portal systems of the right and left liver lobes set the scene for the advance arriving from Japan in 1984 when Makuuchi described the effects of portal venous embolization (PVE) in human clinical settings (35).

Other more recent advances in liver surgery include the ligation of the portal vein during liver resections in one lobe followed by later hemi-hepatectomy and the application of laparoscopy to liver resections $(25,36)$. Finally, a new concept that combines liver

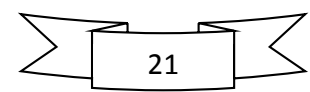


parenchymal transection with portal venous ligation, associating liver partition and portal vein ligation for staged hepatectomy (ALPPS), has emerged and has been demonstrated to stimulate rapid hypertrophy of the future liver remnant (FLR) (37).

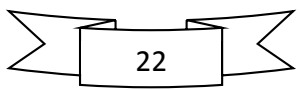




\section{Surgery for colorectal liver metastasis (CRLM)}

Malignant tumors in the liver can be classified as primary malignancies (mainly hepatocellular carcinoma, cholangiocarcinoma and gallbladder carcinoma) and secondary malignancies (metastases). Metastases from cancers of the colon and rectum are historically most relevant to liver surgery, but during recent years, metastases from other solid have been recognized as an indication for liver surgery (38). Currently, surgery is the only treatment that offers a reasonable chance of cure for malignant liver tumors.

In Sweden, CRLM is by far the most common indication for liver resection. Over 14 years, the number of liver resections in Sweden has evolved from approximately 150 to approximately 800 (figure 6). A similar trend is noted in other countries (39-41).

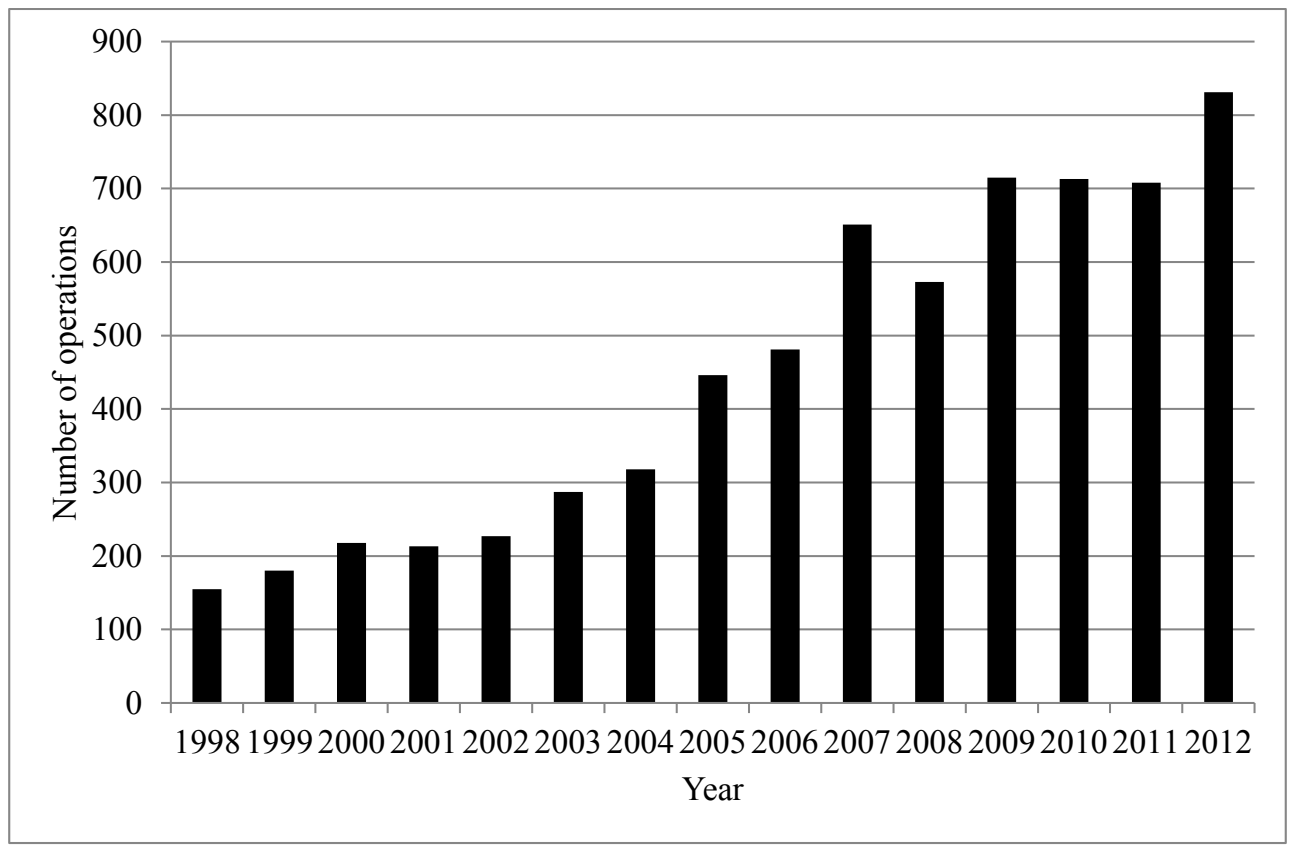

Figure 1. Number of liver resections (JJBxx) in Sweden in 1998-2012. The number of liver resections performed in Sweden each year has increased approximately 500\% over a 14-year period. Data from the Swedish National Inpatient Register (www.socialstyrelsen.se).

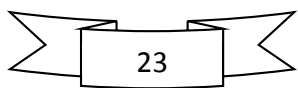


Several explanations for this dramatic increase are available. Before the introduction of active chemotherapy and surgery for CRLM, the prognosis of patients with the disease was dismal, with a 3 -year survival rate below 5\% (42). The high operative risk in the 1970s and early 1980s likely contributed to the strict criteria for metastatic liver surgery (43-45).

In addition, the high risk of relapse after surgery also contributed to the reluctance to perform major liver surgery (45). With the progress of the oncological treatment, the number of patients converted from a disease state not amenable for surgery to a state possible to treat with surgery has increased. Furthermore, the risk for relapse has also been reduced with better patient selection (46-48). In addition, technical advances in both surgery and anesthesiology have increased the safety of liver surgery (49).

Today, the perioperative mortality in liver surgery is as low as $1 \%$, although the criteria for surgical resections have expanded substantially (50). In addition, the 5-year survival of patients treated surgically and oncologically for CRLM has increased to $55 \%$ in large centers (51). 


\section{Liver anatomy and nomenclature}

The liver is the largest solid organ of the human body. A normal adult human liver weighs approximately $2-3 \%$ of the total body weight and lies beneath the diaphragm in the upper portion of the abdomen (52).

The liver possesses ligaments that attach to its surroundings (figure 2). In the midline, the teres ligament contains the remnants of the umbilical vein and continues as the falciform ligament, which attaches between segments IVa and IVb and segments II and III, respectively. Further back along the superior midline, the falciform ligament spreads out and becomes the coronary ligament (figure 2). On the right side, the coronary ligament continues to the bare area of the liver (area nuda hepatis), and lateral to the bare area the right triangular ligament is found (figure 3). On the left side, the coronary ligament continues towards the left triangular ligament laterally.

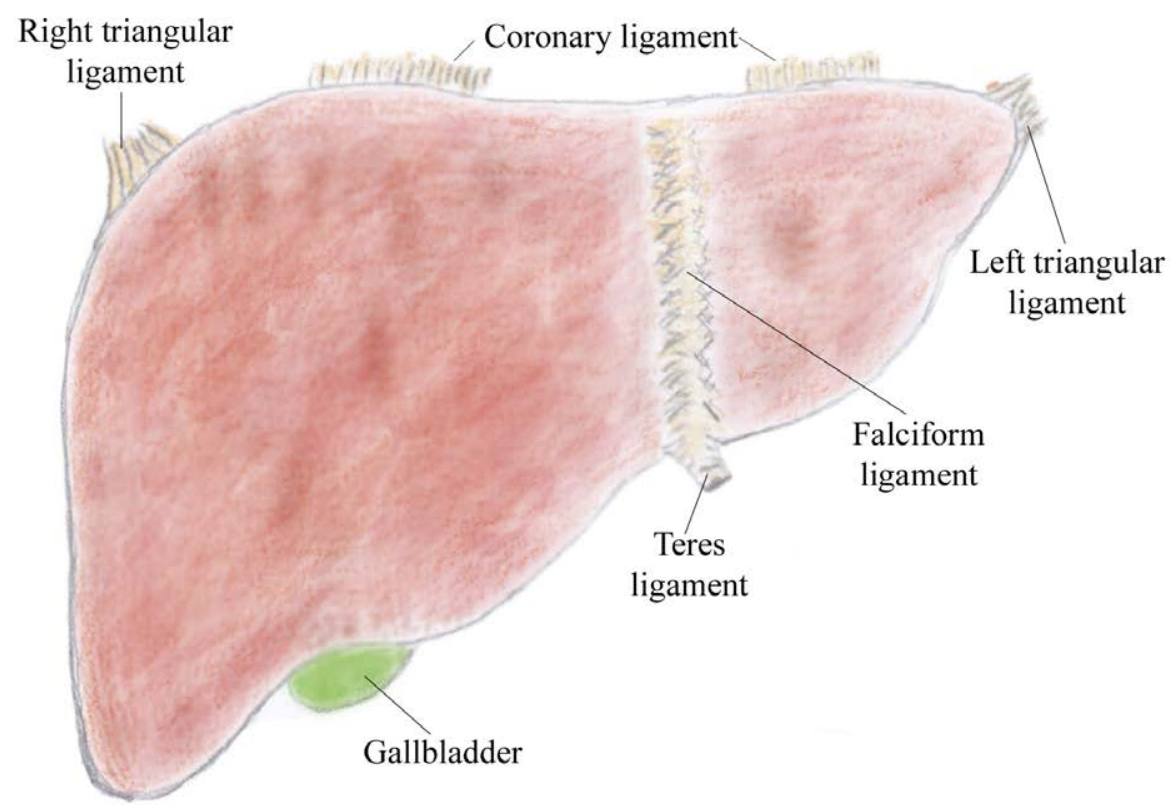

Figure 2. Surface anatomy of the liver, anterior view.

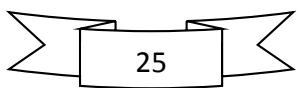


The inferior caval vein runs behind the liver (in a groove or sulcus), and the liver veins join the inferior caval vein before the vein enters the thoracic cavity through the diaphragm. The structures of the hepatoduodenal ligament (the portal vein, the common hepatic duct and the liver arteries) run into the liver hilum.

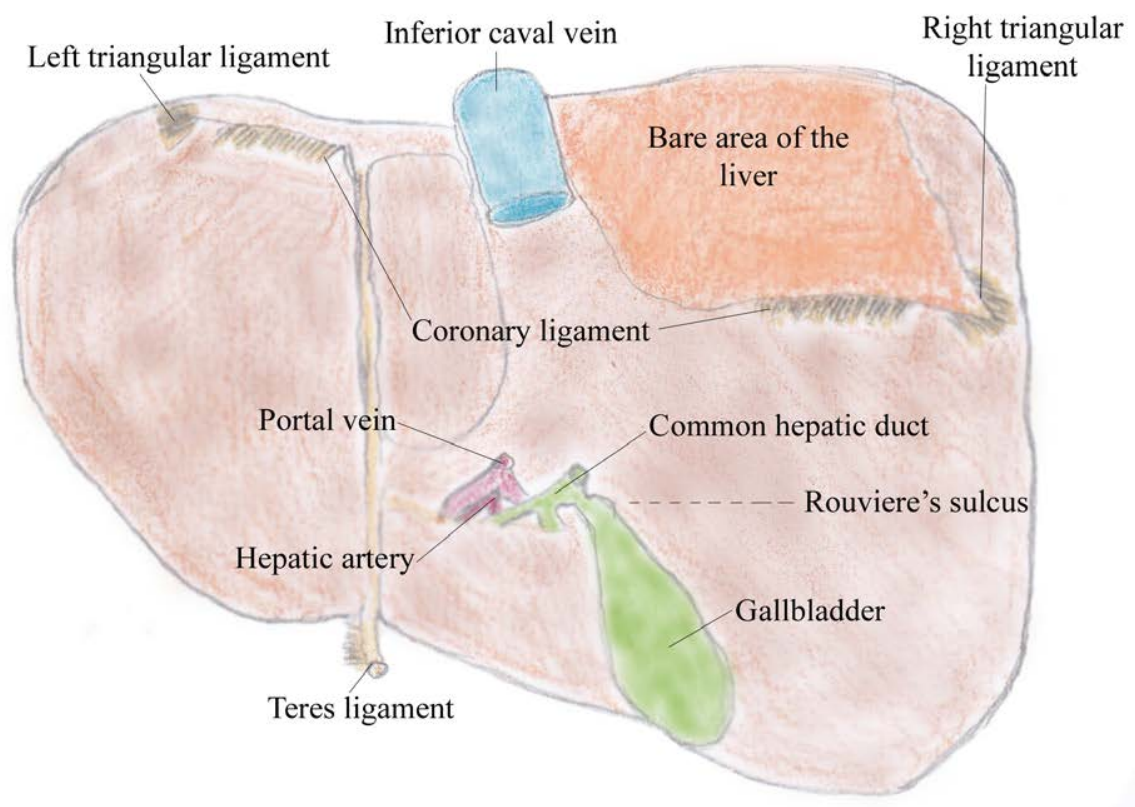

Figure 3. Surface anatomy of the liver, inferior (visceral) view.

On the liver surface, there are few landmarks that can reveal the liver's internal anatomy. Among these landmarks is the Rouviere's sulcus, named after Henri Rouviere, a professor of anatomy and embryology at the University of Paris (53). This cleft in the liver tissue runs to the right of the liver hilum and corresponds to the plane of the right portal pedicle within the liver (figure 3). Another landmark (that typically cannot be seen) on the liver surface is Cantlie's line, which runs from the fundus of the gallbladder and upwards to the center of the caval vein (10). This line (or plane as it follows the caval vein posteriorly) divides the liver anatomically into the right and left lobe (figure 4). Another important landmark on the liver surface is the attachment of the falciform ligament and the fissure to create the "ligamentum venosum" that runs on the inferior surface of the liver. Corresponding to this fissure, the portal pedicle to the anatomical left liver lobe can be found inside the liver.

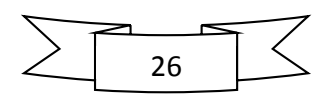


Despite early studies on internal liver anatomy and the distribution of bile ducts, no accepted classifications systems used to describe the liver were formalized in the first half of the $20^{\text {th }}$ century $(54,55)$. The most widely used systems, dating back to the $1950 \mathrm{~s}$, are those of Couinaud, with modifications by Bismuth; Healey and Schroy; and Goldsmith and Woodburne, referred to as the Anglo-Saxon system (26, 27, 56, 57). According to Couinaud, the liver is divided into 8 segments based on the third-order distribution of the portal vein branches. Goldsmith and Woodburne suggested a similar division into 4 segments (each with 2 sub-segments) based on the second-order bile duct and liver artery branches (figure 4, table $1)$.

Both classification systems utilize the distribution of hepatic veins. Each segment has its own inflow, both arterial and portal, as well as bile drainage (figure 4). The three hepatic veins drain most of the blood to the inferior caval vein. The right hepatic vein runs between segments VI and VII and segments V and VIII, respectively; the middle hepatic vein runs between segments V and VIII and segments IVa and IVb, respectively; and the left hepatic vein runs between segments IVa and IVb and segments II and III, respectively (figure 4). In addition, a variable number of short liver veins drain directly into the inferior caval vein. Segment 1 (caudate lobe) is drained by these short veins.

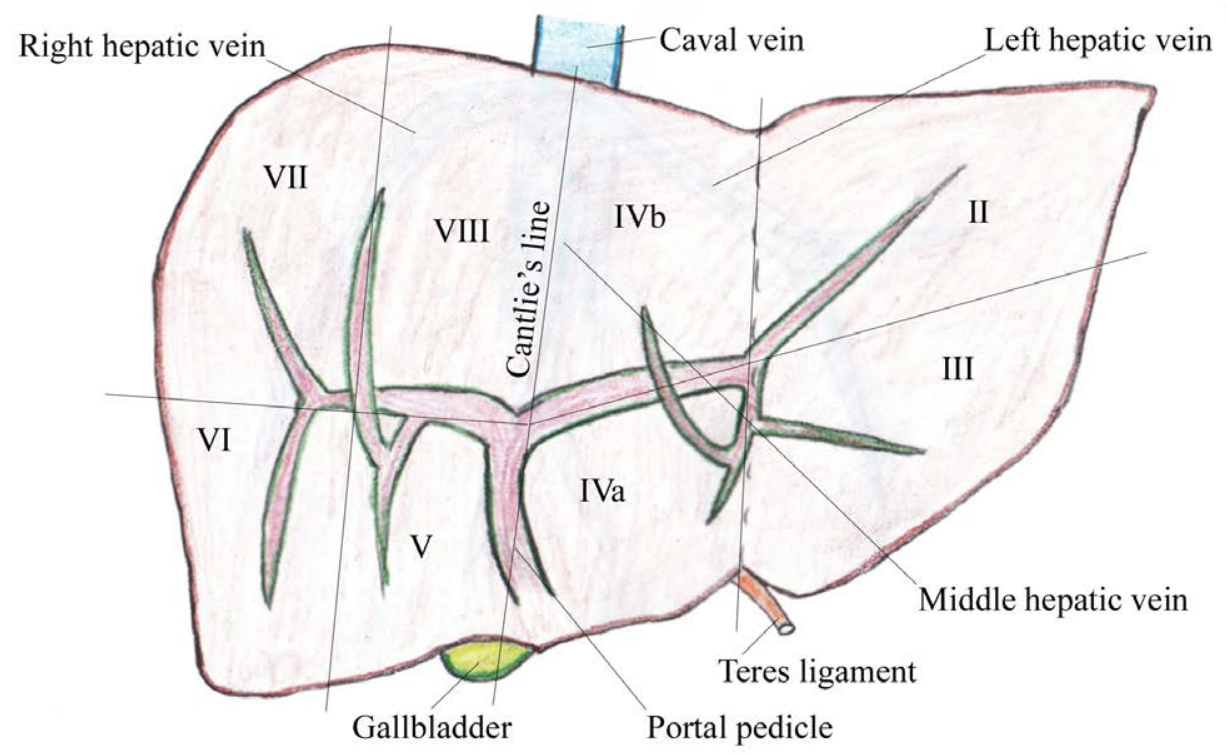

Figure 4. Internal anatomy of the liver. Roman numerals indicate segments.

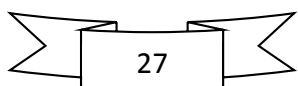




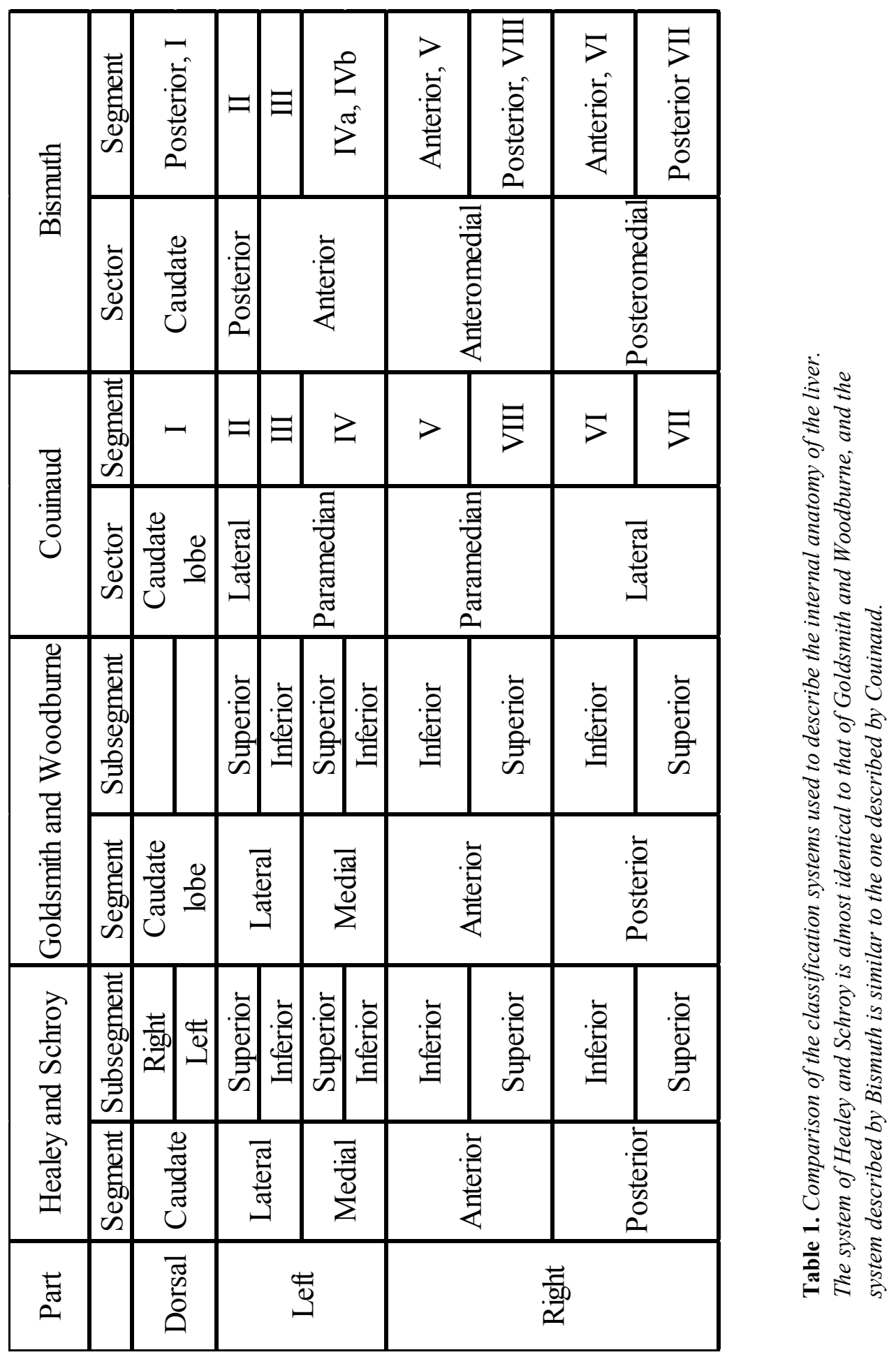

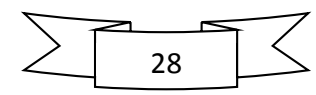


Although the basic division of the liver into segments is generally agreed upon, the nomenclature used to describe various types of anatomical liver resections is not uniform (58). Two systems are the most widely used: in Europe and Japan, resections are primarily described according to Couinaud's system, and in the USA, the Anglo-Saxon system is used. The use of different systems to describe surgical procedures may lead to confusion and misinterpretation. At a meeting in Brisbane in 2000, the leading liver surgeons of Europe and the USA attempted to synchronize the nomenclature to avoid confusion. This is particularly important, as some of the names involved sound very similar (section and sector). This unification resulted in a system based on the course of the hepatic artery and the bile ducts that at least provides a translation for those terms. Although some confusion remains, the Brisbane 2000 terminology is gaining acceptance among liver surgeons (59-61). The terminology divides the liver into right and left (hemi-liver, first order). The right hemi-liver is divided into anterior and posterior sections, whereas the left hemi-liver is divided into medial and lateral sections. Each section consists of 2 segments, wherein segments II and III comprise the lateral left section, segments IVa and IVb comprise the medial left section, segments V and VIII comprise the anterior right section, and segments VI and VII comprise the posterior right section. This division forms the basis for the description of resections. 
$\sum[30]$ 


\section{Techniques of anatomical liver resections}

The article by Professor Henri Bismuth, "Surgical anatomy and anatomical surgery of the liver", described the relevance of earlier anatomical studies of the liver. The internal anatomy of the liver was described from a surgical standpoint, including how individual segments or a group of segments ( 2 or more) can be resected without significantly interfering with vascular structures (27).

During the century since the beginning of liver surgery, much has changed in liver transection techniques. Despite these changes, the "finger fracture" technique with some modifications (mainly the use of clamps instead of fingers, referred to as the Kelly clamp crushing technique or Kelly-clasia) remains widely used (62). The Cavitron Ultrasonic Surgical Aspiration device (CUSA, Tycho Healthcare, Mansfield, MA) is another widely used method.

The CUSA is an ultrasound generator that also incorporates a suction device. The CUSA was first reported in the context of liver surgery in 1980 and has since gained considerable popularity (63). The vibrations at the tip of the CUSA generated by the ultrasound destroy the liver cells, leaving vessels and bile ducts intact. The destroyed cells are sucked up simultaneously, and the vessels and bile ducts are closed by the same methods used for these structures during the crush technique (suture, ligature, clips and diathermia). Numerous reports have been written comparing methods for liver resection with diverging results (64).

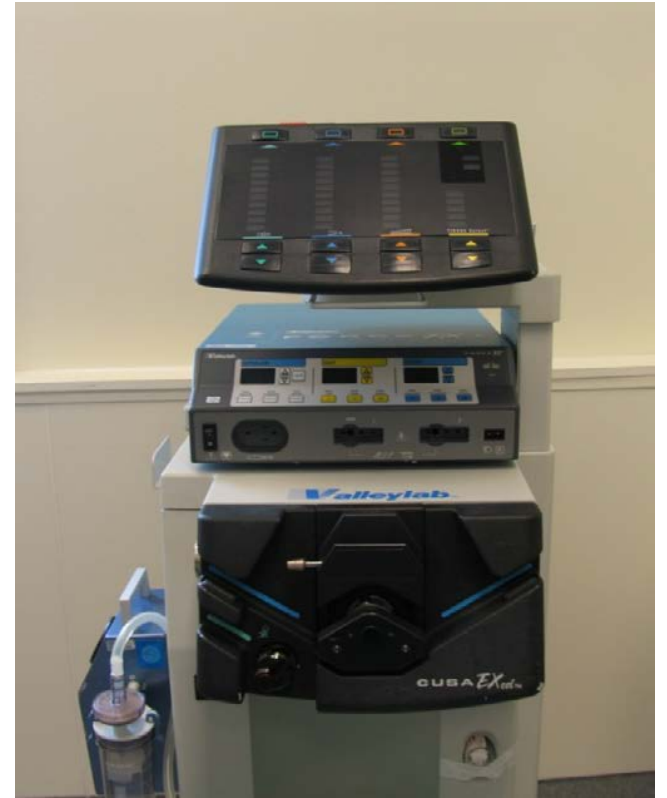

Figure 5. Cavitron Ultrasonic Surgical Aspirator (CUSA). 
Unfortunately, none of these studies included a sufficient number of patients to draw firm conclusions regarding the superior technique $(62,64-67)$. In one study, the crushing method was accompanied by pedicle clamping, whereas the other methods (CUSA, radiofrequency, water jet) were not, making comparisons even more complicated (66).

In addition, there has been an explosion of other methods. These methods include the Ultracision harmonic scalpel (Ethicon), LigaSure (ValleyLab), TissueLink (Dover, NH), Habib sealer and other radiofrequency techniques, such as Cool-tip (Raduibucs, Tyco Healthcare), Hydro-Jet (Hydro-Jet, Erbe, Tubingen) and various stapling instruments. Although none of these methods has gained as much popularity as the CUSA and the crushing technique, all are used to some degree (62).

The ultrasonic scalpel (Ultracision Harmonic Scalpel, Ethicon Endo-Surgery) was introduced in the early 1990s. The ultrasonic technology is used to cut tissues and simultaneously seal the cut edge. The technique has gained increasing popularity with the introduction of laparoscopic liver surgery, but the advantage of this transection technique remains unclear $(68,69)$.

LigaSure was first reported in liver surgery in 2001 (70). LigaSures are bipolar diathermy forceps that claims to effectively seal vessels up to $7 \mathrm{~mm}$. A comparison with other methods of transection provided diverging results $(71,72)$. However, this device has become increasingly popular with the increase in laparoscopic liver surgery (73).

TissueLink, Habib sealer and Cool-tip use radiofrequency energy to generate coagulation. The electrodes are inserted in the transection plane serially, and energy is applied to create coagulation. Subsequently, the tissue can be divided. Although it is claimed to be highly effective in achieving hemostasis, this result has not been proven (74-77).

Hydro-jet is a water propulsion dissector that uses a water jet to fragment the liver parenchyma and expose vessels and bile ducts. This method reduced bleeding compared with CUSA and the crush technique in a retrospective non-randomized study, but these findings were not confirmed in a prospective randomized trial $(66,78)$.

Regardless of what instrument is used to divide the liver parenchyma, anatomic liver resections are those in which the parenchymal division follows the functional anatomical lines of the liver. For hemi-hepatectomies (removal of the right or left hemi-liver), the vascular inflow to the part of the liver to be removed can be divided before parenchymal transection. The division of the portal pedicle can be achieved in an extra-hepatic manner, where the

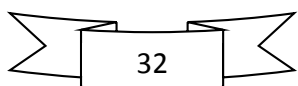


corresponding hepatic artery and portal vein are isolated and ligated in a method described by Lortat-Jacob (22). Another approach for liver resection is intrahepatic ligation (Glissonian approach); for right hemi-hepatectomy, an opening is created in the liver capsule (Glisson's capsule) in the gallbladder fossa (segments $\mathrm{IVb} / \mathrm{V}$ ), and another opening is made to the right and posterior to the right portal branch. A vascular clamp is passed between the openings, and upon closure, the demarcation confirms its placement around the right portal pedicle. Subsequently, a stapling device is used to seal and divide the right portal pedicle. Another variant of the Glissonian approach involves dissecting the hilar plate and identifying the right anterior and the right posterior portal pedicles that then can be ligated and divided separately close to the liver. Damage to structures supplying the left hemi-liver can be avoided by following this procedure.

For left hemi-hepatectomy, the corresponding procedure involves opening the liver capsule at the umbilical fissure above the hilar plate and on the posterior aspect of segment II. Passing a vascular clamp between the openings will isolate the left portal pedicle, which can then be divided. Regardless of the method used to close the inflow to the portion of the liver to be resected, a demarcation appears along the line of anatomical division between the right and the left hemi-liver. Dividing the liver at this line will result in an anatomically correct hemi-hepatectomy.

In a previous study, the Glissonian approach did not result in more complications than the classic extra-hepatic approach in the settings of right hemi-hepatectomy (79). Although that study included a substantial number of patients, the study was not randomized. In addition, a substantial patient selection towards smaller tumors not close to the liver hilum was noted in the group with intraparenchymal division of vessels. The PM length was also significantly longer in that group. Retrospective studies have demonstrated that patients with intrahepatic division exhibit significantly reduced blood loss, fewer complications and less mortality as well as an increased frequency of R0 resections (resections where the histological examination reveals that the whole tumor has been removed) $(80,81)$. However, in these studies, significantly more liver resections (wedge and further segmentectomies) were performed in patients with extra-hepatic vessel control, and extended hepatectomies also frequently occurred in this patient group. Therefore, it might be prudent to adapt the method to the proximity of the tumor to the hilum given that radical removal of the tumors is of the utmost importance.

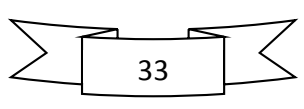


When individual segments or sections (2 adjacent segments) are to be removed, the inflow is located within the liver tissue; thus, the parenchyma must be divided somewhat before the inflow to the part to be resected is selectively divided. On the left side, the portal pedicle is identified in the fissure of the ligamentum venosum, and the branches to segments $\mathrm{IVb}$ and III can often be reached without significant parenchymal dissection, whereas the branches to segments II and IVa are observed in a more cephalic direction, necessitating further parenchymal dissection before these branches can be approached. When dealing with the medial section of the left hemi-liver (segments IVa and IVb), it should also be kept in mind that variable contributions from the right hemi-liver can exist.

The branches to individual sections (anterior and posterior) and segments in the right hemi-liver can typically be reached through dissection of the liver hilum (lowering of the hilar plate) and can thus be divided before parenchymal transection.

When liver resections are performed, another alternative is the so-called atypical liver resection. The main difference between anatomical and atypical resection is that the latter does not follow the anatomical lines of the liver. Atypical resection makes it possible to spare the liver parenchyma that is not affected by the tumor. The goal of atypical resection is to resect all tumors with sufficient margin of tumor-free tissue and to leave as much liver parenchyma as possible. Specific vascular inflow control is not achieved before parenchymal dissection, but the vessels and bile ducts are closed during the division of the parenchyma. Methods of vascular occlusion may be applied in a manner similar to those employed for anatomical resections. The necessary margin has been a matter of research; although a wide margin $(>10 \mathrm{~mm})$, as recommended in the early days of modern liver surgery, may remain desirable, later studies have reported that even resections with smaller margins are sufficient in patients with colorectal liver metastasis (CRLM) (82-85). In the setting of HCC, greater surgical margins are proposed, as these tumors spread within the liver and as micrometastases that are typically present within $2 \mathrm{~cm}$ of the primary tumor are common (86). Altogether, 2$\mathrm{cm}$ surgical margins should be pursued at a minimum, and theoretically, anatomical resection involving the affected segment may be superior to atypical resections (86). This suggestion has indeed been demonstrated in a randomized controlled trial comparing $1-\mathrm{cm}$ and $2-\mathrm{cm}$ surgical margins (87).

Anatomical and atypical resections for CRLM have been compared, and the results have varied between better results for anatomical resection and no difference between the two approaches $(88,89)$. The liver surgeon should be able to choose the type of resection that

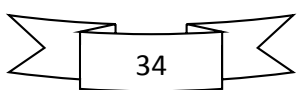


offers the best overall results for the patient. This resection may indeed often necessitate the application of both anatomical and atypical resections.

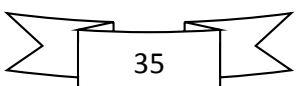


$\sum\left[{ }_{36}\right]$ 


\section{Methods to control bleeding during liver surgery}

In the early days of modern liver surgery, the perioperative mortality of liver resections ranged from 10 to $25 \%$ (43). A significant proportion of this mortality could be attributed to the inherent risk of bleeding. Currently, the perioperative mortality is less than $4 \%$ for liver resections performed for primary hepatobiliary disease and approximately $1 \%$ for other resections performed in centers performing more than 15 resections per year $(50,90)$. This reduction, despite more complex operations in older patients with more advanced diseases, can be largely attributed to a more systemic approach to the subject of hemostasis $(90,91)$. Apart from the obvious risk of exsanguination and death during surgery, transfusions have been related to increased morbidity, mortality and length of hospital stay in a dose-dependent manner (92).

The methods to decrease bleeding during liver surgery can be categorized as surgical methods and anesthesiological methods (or non-surgical methods).

As with all other wounds, much of the bleeding from the liver can be temporarily stopped or at least decreased by direct pressure and thereafter treated with sutures or other general methods of hemostasis. However, this approach is occasionally inadequate to control bleeding from larger vessels within the liver.

The first method specifically developed for liver surgery was the method described by Pringle (16). PM includes the isolation of the hepatoduodenal ligament, which is achieved by entering the foramen of Winslow (behind and to the right of the ligament) and the lesser omentum (on the left side of the ligament), thus giving the surgeon access to the ligament from all sides. The second step is to apply pressure to the ligament (either with a vascular clamp or a band), thus occluding the common hepatic artery, the portal vein and the common bile duct. Although this approach occludes both parts of the double circulation to the liver, it does not greatly affect bleeding from the liver veins. 


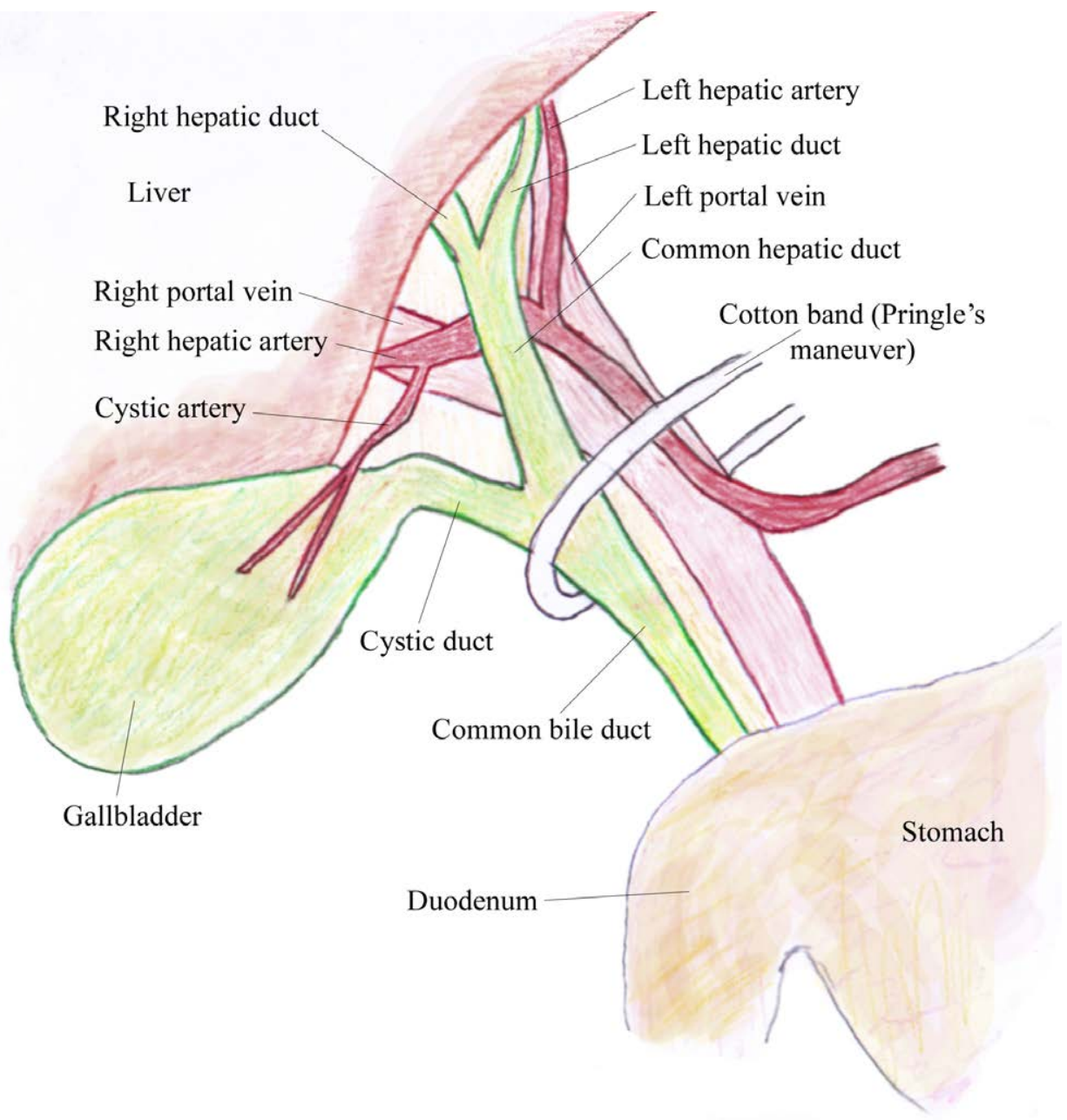

Figure 6. Pringle's maneuver. A cotton band or vascular clamp is placed around the hepatoduodenal ligament to close the inflow (hepatic artery and portal vein) to the liver.

Total hepatic vascular exclusion (THVE) is another surgical method for reducing bleeding that is unique to liver surgery. In addition to the isolation of the hepatoduodenal ligament, the caval vein is isolated both inferiorly and superiorly to the liver. The accessory arterial supply to the left liver lobe from the left gastric artery is isolated. The inferior caval vein is clamped followed by clamping of the hepatoduodenal ligament and the accessory left liver artery; finally, the inferior caval vein is clamped superior to the liver. The clamping of the inferior caval vein inferior to the liver veins is either performed above the right adrenal vein, or the right adrenal vein is ligated. When applied for prolonged periods, hypothermic perfusion may decrease the adverse effects on the liver (93). This method has been compared

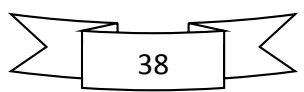


with PM, and both techniques reduce bleeding compared with no vascular occlusion. THVE, however, carries a considerably increased risk for hemodynamic instability, and its routine use is not advocated (94-96).

Selective hepatic vascular exclusion (SHVE) is another technique that has the advantages of total hepatic exclusion without the hemodynamic consequences. Instead of clamping the caval vein inferiorly and superiorly to the liver, the liver veins are clamped selectively (97). Thus, the flow in the inferior caval vein is not disrupted, and the risk of hemodynamic instability is reduced (98). With regard to reducing blood loss, SHVE is equally effective as THVE and is more effective than PM $(98,99)$. This method resembles a technique widely used for hemi-hepatectomies in which both the vascular inflow and the involved liver vein are divided before parenchymal transection (see previous chapter); however, SHVE also involves occlusion of the other liver vein(s) and the entire hepatoduodenal ligament. This approach may be indicated when tumors are located in close proximity to the liver veins as well as in patients with raised central venous pressure (CVP).

In addition to the surgical methods, various pharmacological agents can be used to achieve hemostasis. However, these aspects of liver surgery are beyond the scope of this book. In addition, some of the methods used to divide the liver parenchyma have claimed to reduce bleeding (see previous chapter).

The main anesthesiological method to reduce bleeding during liver resections is low CVP anesthesia, which is targeted towards bleeding from the liver veins. The rationale behind this approach is that the liver veins drain directly into the caval vein, and thus, the pressure in the liver veins is roughly the same as in the caval vein. This concept became popular in the 1990s, and early reports suggested significantly reduced bleeding with low CVP anesthesia compared with anesthesia without specific measures to reduce the CVP (49). To achieve the goal of low CVP, between 2 and $5 \mathrm{~mm} \mathrm{Hg}$ fluid restriction is applied before and during the surgery (until the transection of the liver is complete). In cases where this approach alone is insufficient, intravenous nitroglycerine is used to further reduce the CVP. A central venous line is necessary to monitor the patient. This approach minimizes the distention of hepatic veins and sinusoids, thus reducing "back bleeding". Towards the end of the 1990s, this strategy was shown to be effective in non-randomized studies $(100,101)$. Later, small randomized studies summarized in a meta-analysis confirmed this finding (102). This approach is expected to reduce bleeding by up to $50 \%(49,102)$.

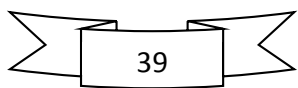


No treatment is without risks or side effects, and low CVP, the "controlled hypovolemic state", is no exception. This hypovolemia can cause inadequate perfusion, and in the event of sudden profuse bleeding, the volume reserve can be minimal. The kidneys are the organ system that is most likely to be affected and the most accessible. However, in one of the early studies, the incidence of renal failure was not increased with low CVP anesthesia (101).

THVE (see above) carries some anesthesiological implications given that it typically cannot be applied to a patient with (controlled) hypovolemia. For THVE, the CVP generally must be in the upper range, typically above $15 \mathrm{mmHg}$. This CVP allows the clamping of the inferior caval vein, which can decrease venous return and cause a sudden decrease in cardiac output as well as increased afterload. By maintaining a high CVP, clamping can be performed without jeopardizing adequate blood pressure and circulation in most cases. In some cases, vasoactive agents are needed in combination with the volume load to maintain the perfusion, and it should be kept in mind that if adequate volume load and the use of vasoactive agents fail to provide acceptable perfusion, veno-venous bypass may be required.

Autologous blood donation, hemodilution and hypoventilation are other non-surgical methods for reducing blood loss in liver surgery. However, none of these methods are regularly use in the clinic; therefore, these techniques are not included in this review. 


\section{Obstacles in liver surgery}

Given that numerous obstacles, such as general anesthesia, the understanding of liver anatomy, the identification of methods for transecting liver tissue and bleeding control during liver surgery, have been solved, one might imagine that all obstacles to liver surgery had been removed. Unfortunately, further work is needed. In the 1980s and early 1990s, the criteria for resecting metastases from colorectal cancer in the liver included metachronous detection of the liver disease, no more than three metastases restricted to one of the liver lobes, no metastases greater than $5 \mathrm{~cm}$, a possible resection margin of $1 \mathrm{~cm}$ and no signs of spreading outside the liver (103). These criteria were largely due to the prognosis of the malignant disease but also to the operative risk associated with more extensive operations.

With the implementation of modern chemotherapy, the prognosis for CRLM improved, and the indications for liver resections have broadened ever since. Today, extra-hepatic disease is not an absolute contraindication to liver surgery as long as the disease is treatable (104). The number and the distribution of liver lesions are not seen as a contraindication per $s e$, but it is generally agreed that liver tissue corresponding to $20 \%$ of the liver must remain intact and circulated, provided that the patient has not received chemotherapy (105). This is one of the obstacles in liver surgery not likely to be overcome in the near future given that liver failure will result from more extensive resections.

Another challenge is that patients who initially possess unresectable liver metastases exhibit approximately the same prognosis as those who present with resectable disease if unresectable patients are successfully treated with chemotherapy before surgery and the disease is "down-sized" to a resectable situation (47). This challenge leads to a growing population of patients scheduled for liver surgery who have been heavily treated with chemotherapy before operation, which may decrease the quality of the FLR and increase the risk for complications (106). For patients undergoing operations after chemotherapy, a FLR of $30 \%$ has been suggested as a threshold due to the reduced functional reserve of the liver (107). In the setting of liver fibrosis or cirrhosis, which is common in HCC and may be present in CRLM as well, a 40\% margin has been proposed (107).

To further complicate this situation, the operations need to achieve R0 or at least R1 status, are often technically demanding and might increase the usage of PM, thereby subjecting the FLR to IRI. In addition, the population of patients to be treated with

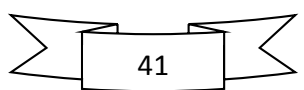


chemotherapy and liver surgery is growing older. Although chronological age per se is not considered a contraindication for surgery, the physiological reserve of the elderly with stage IV cancer (cancer with distant metastases) can be expected to be further reduced (91).

Operations for recurrent liver metastases from colorectal cancer exhibit a similar survival rate as first-time operations $(108,109)$. However, this approach will increase the intraoperative bleeding and will be demanding, both for the surgical team as well as the patient (108-110). Furthermore, additional obstacles might develop in a special situation wherein a two-stage operation is planned after initially unresectable metastases have responded to chemotherapy. 


\section{Ischemia/reperfusion injury (IRI)}

Given that the surgical methods used to reduce bleeding in liver surgery potentially involve closure of the circulation to the liver, some degree of ischemia cannot be avoided. When the circulation is restored, reperfusion will occur. This combination and its consequences have been referred to as ischemia/reperfusion injury (IRI). Although concerns about hepatic inflow stasis were raised in the literature in 1963, the first publications regarding hepatic IRI date only to the late 1970 s and early 1980 s, although the topic has recently gained increasing interest (111-114).

IRI is an ill-defined injury after a period of ischemia followed by reperfusion with oxygenized blood. In the liver, IRI has historically been defined by the tissue damage observed after prolonged ischemia and reperfusion. The markers classically used to describe the severity of IRI include the liver enzymes, aspartate aminotransferase (AST) and alanine aminotransferase (ALT), as well as histological signs of tissue damage. Given the multifactorial nature and complexity of IRI, these markers may be regarded as insufficient methods. Currently, IRI is recognized as a complex cascade of events initiated by the ischemic insult. The reperfusion phase starts with the return of oxygenized blood and can be divided into early ( $<2$ hours) and late ( 6 to 48 hours) reperfusion; however, the division is somewhat arbitrary and leaves a window of 4 important hours $(115,116)$. The injury leads ultimately to both necrosis and apoptosis in the liver.

Upon ischemia, the oxygen tension in the tissue will decrease, and the metabolism will change from physiological aerobic metabolism to anaerobic metabolism. As a consequence, the production of phosphorylated high-energy compounds (adenosine triphosphate, ATP) decreases, ultimately becoming insufficient for cellular metabolism of hepatocytes, sinusoidal endothelial cells and Kupffer cells (117). The ATP deficiency causes a loss of cellular membrane ion pump function, resulting in increased intracellular concentrations of sodium and calcium ions. This loss of function in turn causes cells to swell, and the increased concentration of intracellular calcium activates phospholipases, which degrade the membrane phospholipids (118). Simultaneously, the tissue becomes acidic, which further increases cellular dysfunction $(118,119)$. With the disruption of cellular membranes, cell contents, such as AST and ALT, begin to leak into the interstitium. The disruption of the cellular membrane and the leakage of cellular phospholipids (phosphoglycerol) forms the rationale for one of the microdialysis measurements further described in chapter 11 (120).

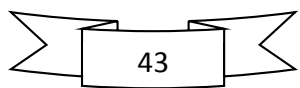


The main cells involved in IRI include hepatocytes, Kupffer cells (macrophages in the liver), sinusoid endothelial cells and polymorphonuclear neutrophils (PMNs).

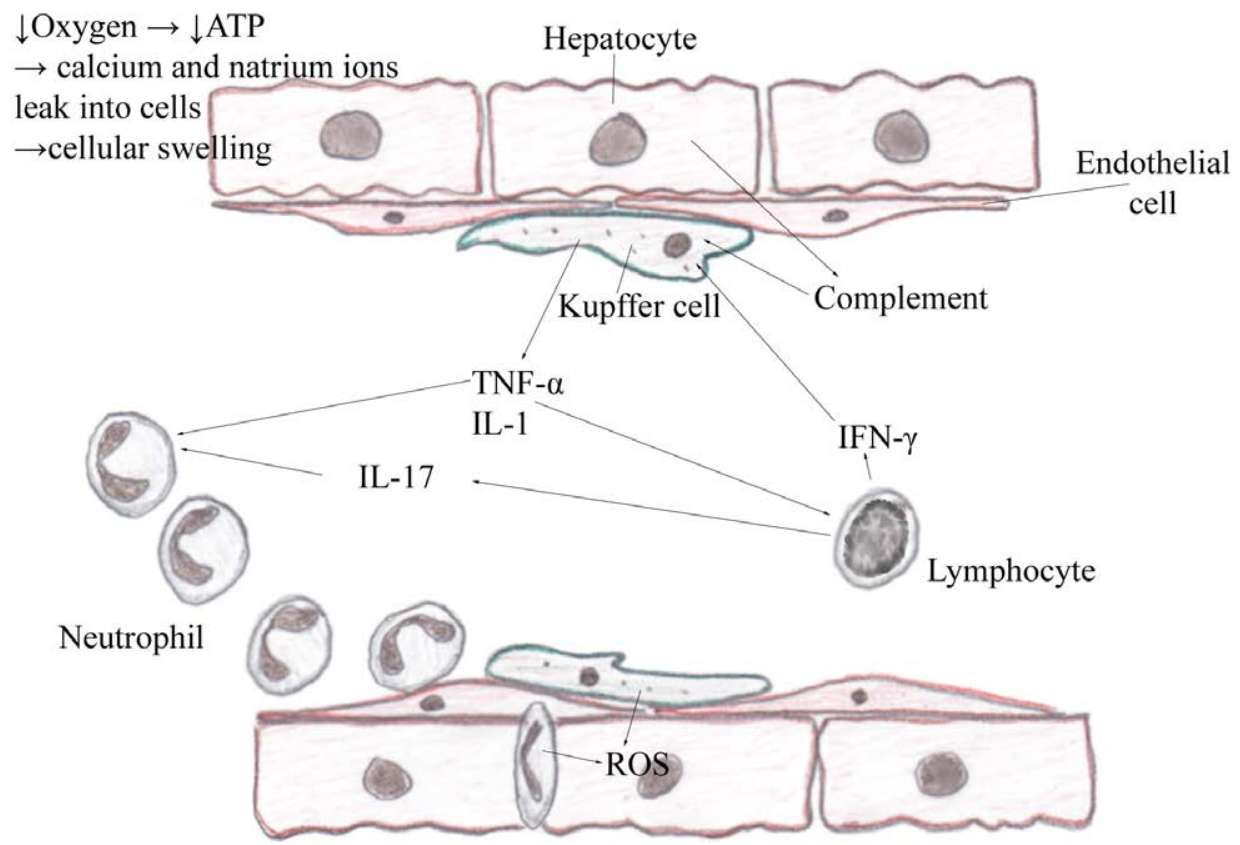

Figure 7. The major cells and mediators involved in liver IRI.

ATP $=$ Adenosine triphosphate

TNF- $\alpha=$ Tumor necrosis factor $\alpha$

IL-1/IL-17 = Interleukin 1/interleukin 17

IFN- $\gamma=$ Interferon $\gamma$

ROS $=$ Reactive oxygen species

The main reactive oxygen species (ROS) involved in liver IRI include the superoxide radical $\left(\cdot \mathrm{O}_{2}{ }^{-}\right)$, hydroxyl radical $(\cdot \mathrm{OH})$ and hydrogen peroxide $(\mathrm{H} 2 \mathrm{O} 2)$. ROS are categorized as radicals and non-radicals depending on the presence (radicals) or absence (non-radicals) of an unpaired electron. Hydrogen peroxide alone is a non-radical but can react to form highly active radicals. The three systems that produce ROS during liver IRI are the nicotinamide adenine dinucleotide phosphate (NADPH) oxidase system, the mitochondrial respiratory chain and xanthine oxidase $(115,121)$.

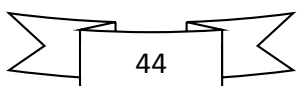


Xanthine oxidase has been the focus of research during the last decades. Xanthine oxidase is an intracellular enzyme that exists as xanthine dehydrogenase under physiological conditions. Upon prolonged ischemia, xanthine dehydrogenase is converted to the ROSforming oxidase (122). Furthermore, the substrates xanthine and hypoxanthine are metabolized relatively quickly and flushed out during reperfusion, whereas accumulation occurs during ischemia (123). Although the inhibition of xanthine oxidase with allopurinol has some effect on preventing IRI, it is seems clear that the enzyme does not play a key role in the cascade (124).

Mitochondria-generated superoxide is another ROS that might be involved in the process of IRI. Again, prolonged ischemia appears to be required for the process to become important in oxidative stress (125). In the mitochondria, a number of protective enzymes, such as superoxide dismutase and glutathione peroxidase, are also present that detoxify ROS to some degree $(126,127)$.

NADPH oxidase is found in both Kupffer cells and PMNs that generate superoxide (128). This superoxide formation has been observed in the settings of hepatic IRI and has been suggested as a viable option in the treatment of IRI $(129,130)$. The inhibition of NADPH oxidase protects against hepatic IRI in mice (131). However, this result has not been observed in clinical studies.

Although hepatocytes are largely considered victims of IRI, they also contribute to the cascade by releasing IL-12. This interleukin may activate inflammatory responses, including TNF- $\alpha$ and IFN $\gamma$ release, in livers subjected to IRI (132). As hepatocytes provide $80-90 \%$ of the complement factors found in plasma, these cells are likely to be responsible for the complement-derived activation of Kupffer cells (see below) observed in hepatic IRI (133).

Kupffer cells play an important role in the early phase of reperfusion and are the main source of ROS generated during that phase (134). The swollen and activated Kupffer cells also release TNF- $\alpha$ and IL-1 early. After a 2-hour delay (reperfusion), increased release of IL6 is observed as well. TNF- $\alpha$ secretion appears to stimulate Kupffer cells to further secrete TNF- $\alpha$ (positive feedback), and inhibition of the IL-1 receptor in Kupffer cells reduces TNF- $\alpha$ production $(135,136)$.

Although TNF- $\alpha$ and IL-1 are cytokines with systemic proinflammatory properties, IL-6 is to some extent anti-inflammatory. IL-6 moderates the inflammatory response and reduces TNF- $\alpha$ expression as well as IRI (137-139).

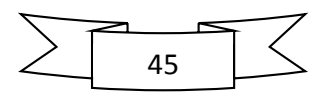


Kupffer cells are also stimulated by complement. Complement-depleted rats respond to IRI with a reduced ALT elevation and less PMN infiltration than rats with intact complement; furthermore, this response appears to occur during the activation of Kupffer cells (140). Some studies have reported that inhibition of the complement system can reduce hepatic IRI (141144). ROS, TNF- $\alpha$ and IL-6 secretion from activated Kupffer cells rapidly (within an hour of reperfusion) attracts lymphocytes to the liver $(145,146)$. The lymphocytes in turn further activate the Kupffer cells to secrete TNF- $\alpha$ with IFN- $\gamma$ release (145). In addition, the secretion of IL-17 by lymphocytes appears to regulate the recruitment of neutrophils (147). In addition to the attraction of lymphocytes and the ROS production that is directly toxic, Kupffer cell secretion influences neutrophil recruitment and activation as well as sinusoid endothelial cells (see below).

The effect of PMNs is noticed later than the effect of Kupffer cells in hepatic IRI and appears to be initiated by the secretions described above $(134,148)$. TNF- $\alpha$ (released by Kupffer cells) has been shown to both activate neutrophils and facilitate their accumulation in the liver via the up-regulation of adhesion molecules (in hepatic post-sinusoidal venules), such as intercellular adhesion molecule-1 (ICAM-1) and P-selectin (137, 149-151). Although these mechanisms are potentially important in the post-sinusoidal venules, the importance of adhesion molecules in the liver sinusoids has been questioned (152). Regardless of the mechanism by which the PMNs accumulate and migrate into the liver (see below), these cells play an important role in the IRI. PMNs are a cell type that have the ability to form ROS. The main source of superoxide formation by PMNs is NADPH oxidase (153). Upon arrival to the liver, PMNs are primed, and the oxidant stress appears after 6-24 hours of reperfusion (148, 154). The PMNs adhere to damaged hepatocytes (similar to the interaction with endothelial cells) via interactions between the beta2-integrin MAC-1 and ICAM-1, the expression of which is stimulated by TNF- $\alpha$ (155). When activated, PMNs produce ROS that diffuse into the hepatocytes and trigger mitochondrial dysfunction. Intracellular oxidant stress might ultimately lead to hepatocyte death (156). The release of proteases (elastase, cathepsin G) is another mechanism by which PMNs kill hepatocytes, but the significance of this mechanism in vivo remains unclear $(157,158)$.

Similar to hepatocytes, sinusoid endothelial cells are largely a target of IRI. However, the role of these cells in the pathogenesis of IRI, which is mediated through interactions with other cells that are attracted to the liver, is important. The sinusoids have been identified as the main site for PMN extravasation into the liver, which occurs without the involvement of

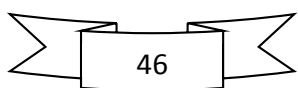


ICAM-1 or P-selectin $(152,155,159)$. It is reasonable to assume that another IRI-related mechanism is responsible for the accumulation and extravasation of PMNs. The initial ATP depletion associated with liver ischemia causes a volume increase in the endothelial cells in a manner similar to that observed in Kupffer cells and hepatocytes (160).

The accumulation of platelets in the sinusoids correlates with reduced sinusoidal perfusion. Furthermore, platelets adherent to the sinusoid endothelial cells (activated by Kupffer cells but independent of P-selectin) induce PMN accumulation in the sinusoids (161, 162). In addition to sinusoidal endothelial cell swelling and platelet adherence, the microcirculation is further impaired by the change in nitric oxide (NO) production (see chapter 8) with respect to endothelin production (figure 8) (163).

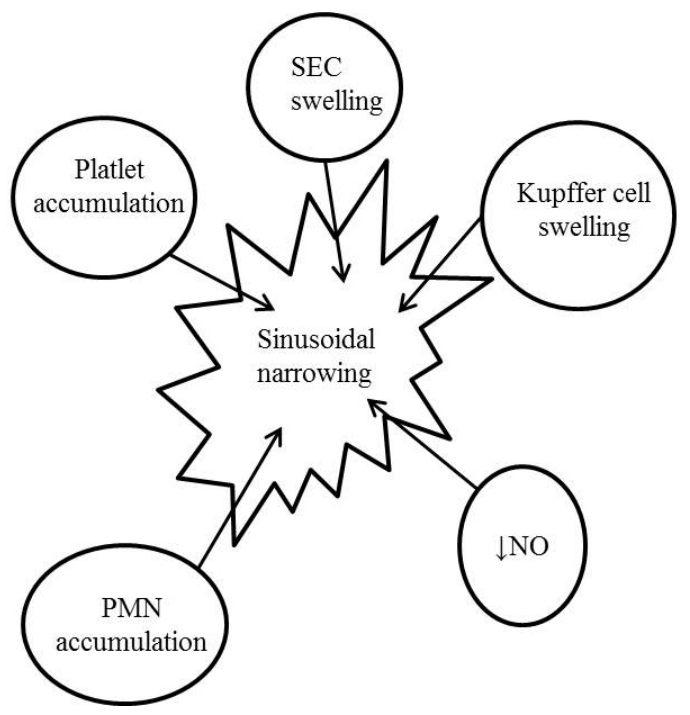

Figure 8. Sinusoidal swelling in liver IRI.

SEC $=$ Sinusoid endothelial cell

$N O=$ Nitric oxide

$P M N=$ Polymorphonuclear neutrophil

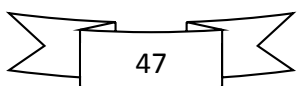


In summary, liver ischemia/reperfusion (IR) leads to the activation of hepatocytes, Kupffer cells and sinusoid endothelial cells. The Kupffer cells produce ROS but also activate lymphocytes and neutrophils. These cells accumulate in the liver via extravasation in the liver sinusoids and are responsible for the later phase of the IRI cascade in which sinusoidal cells and hepatocytes are injured. Figure 9 summarizes the pathways involved in liver IRI.

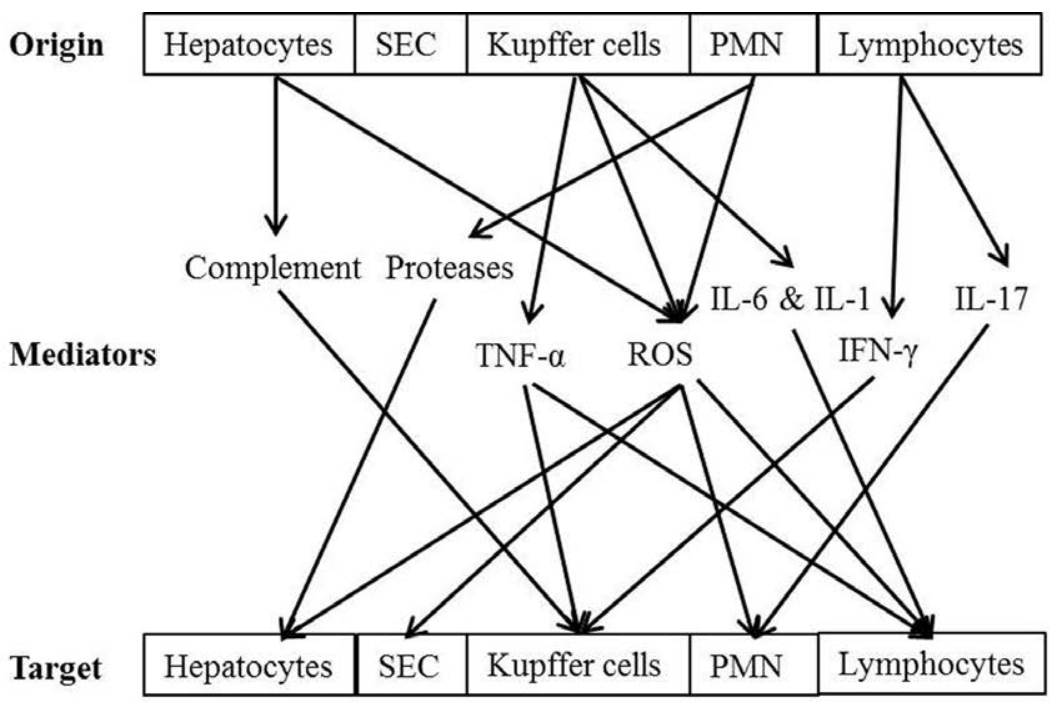

Figure 9. Summary of liver IRI.

$S E C=$ Sinusoid endothelial cell

$P M N=$ Polymorphonuclear neutrophil

IL-1/IL-6/IL-17 = Interleukin 1/6/17

TNF- $\alpha=$ Tumor necrosis factor $\alpha$

ROS $=$ Reactive oxygen species

IFN- $\gamma=$ Interferon $\gamma$

In addition to the detrimental effect of IRI on the FLR, liver ischemia might stimulate the malignant disease that served as the original cause of the surgery. Animal studies have indicated that ischemia stimulates tumor growth, possibly in a dose-dependent manner (164, 165). Furthermore, it has been suggested that selectively clamping the portal vein instead of performing PM might reduce this risk (166). However, to date, no human studies have demonstrated this effect in a clinical setting, possibly due to the multifactorial nature of both the malignant disease and the ischemia applied during liver surgery.

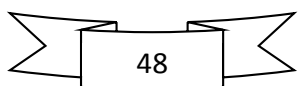




\section{Reactive nitrogen species (RNS)}

The main reactive nitrogen species (RNS) include nitric oxide $(\cdot \mathrm{NO})$ and peroxynitrite $\left(\mathrm{ONOO}^{-}\right)$. Given that it possesses an unpaired electron, $\mathrm{NO}$ is a highly reactive free radical. The discovery of its signaling properties in the cardiovascular system earned three distinguished researchers the Nobel Prize in Physiology or Medicine in 1998. Since then, the role of NO in liver IRI has been studied vigorously, but much remains unclear within the field.

The main source of NO in the human body is endogenously produced by nitric oxide synthase (NOS). NOS has three isoforms: neuronal (nNOS), endothelial (eNOS) and inducible (iNOS). nNOS is almost exclusively found in neural tissue and is therefore outside the scope of this book. eNOS is a calcium-/calmodulin-dependent enzyme that catalyzes the production of NO from the amino acid L-arginine and oxygen (figure 10). eNOS is expressed in liver endothelial cells and hepatocytes $(167,168)$.

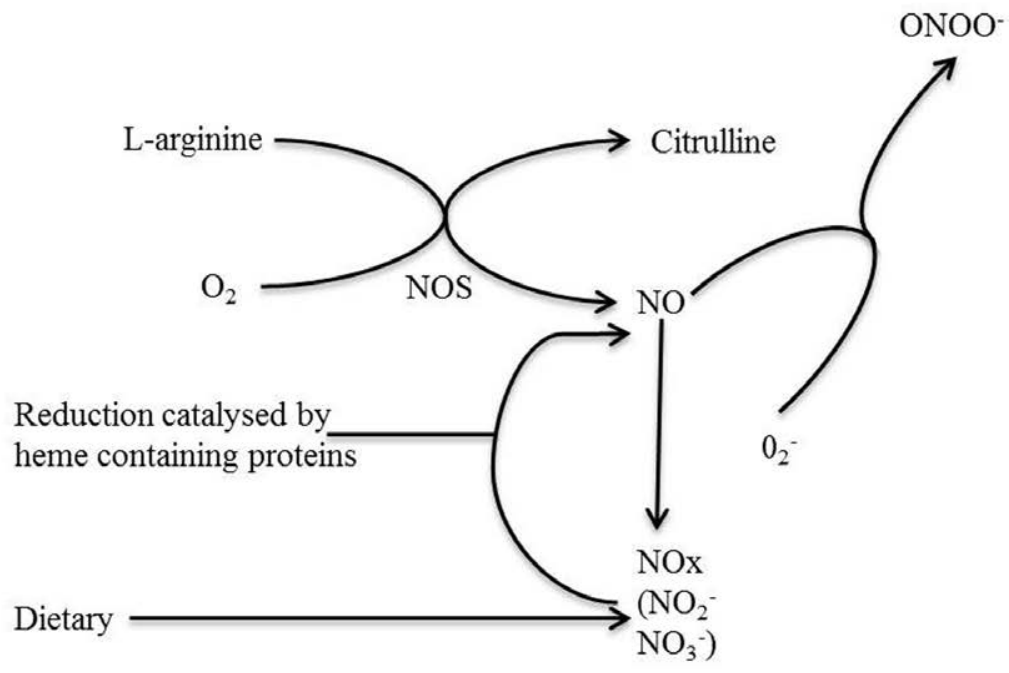

Figure 10. Nitric oxide metabolism. Under physiological conditions, nitric oxide synthase (NOS) produces NO from L-arginine and oxygen. NO is degraded by oxidation to nitrite (NO2-) and nitrate (NO3-) but can also react with superoxide (O2-) to form peroxynitrite (ONOO-). NOx formed from NO and from dietary sources can be reduced to NO.

The third isoform is iNOS, which is bound to calmodulin irrespective of calcium concentrations (calcium-independent). This enzyme is not expressed under physiological

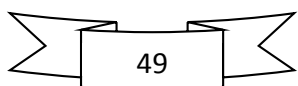


conditions but is up-regulated in many cell types during IRI, including hepatocytes, Kupffer cells and neutrophils (169-171). In the setting of liver IRI, the main source of NO after the upregulation of iNOS appears to be hepatocytes $(169,172,173)$.

Although it is widely recognized that NO plays an important role in IRI, much of the details involved remain unclear. NO relaxes and dilates the liver sinusoids, thus making the perfusion of the liver sinusoids NO-dependent, at least to some degree. This action is opposed by endothelin, and studies have demonstrated that endothelin inhibition can improve the microcirculation in the liver during IRI $(174,175)$. On the other hand, the inhibition of NO production decreases liver circulation (176). Furthermore, it has been suggested that this effect is predominantly exerted through effects on the arterial circulation of the liver given that IRI is not increased when NO production is blocked in the context of common hepatic artery occlusions with intact portal circulation (176). However, NO clearly exhibits some positive effects on the IRI observed with total occlusion of the liver blood supply, as inhibition of NO production by the administration of $\mathrm{N}^{\mathrm{G}}$ nitro-L-arginine methyl ester (LNAME) increases IRI in a rat model of total hepatic ischemia (177). These results potentially reflect the effect of blocking eNOS and thus inhibiting the physiological production of NO. This explanation is further supported by results demonstrating that eNOS-knockout mice experience more severe IRI than wild type mice (172). Furthermore eNOS overexpression decreases IRI in a mouse model (178). The administration of the NO substrate L-arginine immediately prior to partial (hepatic artery only) liver ischemia increases liver blood flow during reperfusion and reduces IRI (179). The source of NO in this treatment is likely to be eNOS. In addition, NO protects endothelial cells during IRI and improves the hepatic microcirculation $(172,180-184)$.

Given that eNOS produces relatively low amounts of NO compared with iNOS, the effects of high NO concentrations have been attributed to iNOS activation when studied in the setting of prolonged (greater than 60 minutes) ischemia. Substantial data from studies assessing iNOS inhibition support the detrimental effect of iNOS activation in both porcine and rat models of hepatic IRI $(171,185-188)$.

NO reacts with superoxide to form the potent oxidant and highly cytotoxic compound peroxynitrite, which might explain the protective effect on IRI observed with iNOS blockade $(186,189)$. In addition, excessive amounts of NO increase leucocyte activation and trafficking into the liver (188).

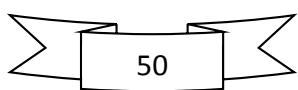


In the setting of short ischemic insults (less than 60 minutes), the effect of iNOS is more controversial. In a study on knockout mice, eNOS was protective in the setting of 1-hour partial hepatic ischemia, whereas iNOS increases IRI (190). A similar study reported increased signs of IRI in eNOS-deficient mice but no such increase in iNOS-deficient mice (191). The length of reperfusion has also been suggested to play an important role in the effects of iNOS as the effect of iNOS varies depending on the length of reperfusion. Threehour reperfusion after 45-minute ischemia resulted in more severe IRI in iNOS knockout mice than wild type mice. However, this result was not observed after 1 or 6 hours of reperfusion (192).

Inhaled NO has been tested in the setting of human liver transplantation and was found to reduce the increase in transaminases associated with the procedure as well as the number of complications $(193,194)$. As the half-life of NO is only a few seconds, the protection observed with NO inhalation in this study may be related to nitrite (195). Nitrite is a relatively stable compound in the human body, thus making nitrite a pool for later NO production by reduction catalyzed by heme-containing proteins (196).

At the cellular level, NO affects the production of ROS and energy in the mitochondria (197). NO inhibits the mitochondrial respiratory chain, thus reducing the release/formation of ROS during reperfusion $(196,198-200)$. Under physiological conditions with a normal supply of oxygen, pyruvate enters the mitochondrial respiratory chain, and ATP is generated. In the process, NO is oxidized to nitrite by cytochrome c oxidase. When oxygen is lacking (ischemia), NO blocks cytochrome c oxidase, resulting in the accumulation of acetyl CoA and the reduction of pyruvate to lactate. Similarly, the amount of NO present during reperfusion might modulate the mitochondria to reduce the burst of ROS as well as ATP production (197). 
$\sum[52]$ 


\section{Ischemic preconditioning (IPC)}

The recommendation of short-acting ischemia followed by reperfusion before a longer-lasting ischemic insult as a means to protect an organ originated from studies on dog myocardium in the 1980s (201). In the mid-1990s, this concept started to spread to liver surgery and has been a topic of intense research during recent years (202). Multiple studies in rodents have demonstrated that ischemic preconditioning (IPC) is effective in reducing IRI in healthy animals as well as in obese animals and those with fatty liver $(183,203-213)$.

Of special interest are studies that report different effects of IPC on aged animals compared with younger animals $(117,214)$. This finding may be increasingly important given that aging increases IRI and as liver resections are more frequently performed on older patients than before (215).

The general hypothesis of the mechanisms underlying IPC is that the induction of lowgrade oxidative stress prepares the cells for the insult of longer-lasting ischemia followed by reperfusion (216).

More specific mechanisms have suggested the induction of antioxidant survival genes, such as heme oxygenase-1 (HO-1) (217). Sinusoidal endothelial cell death decreases with IPC, and this effect appears to be mediated through the adenosine $\mathrm{A}(2)$ receptor pathway (218). Furthermore, it has been suggested that adenosine exerts its effect through the simulation of NO production (219). In the setting of prolonged (75 minutes) total hepatic ischemia, IPC reduces caspase 3 activity and apoptosis (209). Other studies have suggested that additional apoptosis-regulating genes, such as c-jun, are involved in the protective effect of IPC and that IPC reduces transcription and inhibits apoptosis (203, 205, 210, 220). IL-6 is another possible mediator of the IPC effect; however, studies have reported diverging results, including reports of hepatoprotection when IL-6 is administered as well as reports of reduced levels of IL-6 after IPC compared with I/R alone (206, 207). IPC results in lower TNF- $\alpha$ levels than I/R alone, but depleting TNF-/- mice of TNF- $\alpha$ ameliorates the protective effect of IPC $(206,207,213)$. Thus, it appears that a low dose of TNF- $\alpha$ is required for the protective effect of IPC, as previously suggested (221). In addition to these protective mechanisms, IPC induces a regenerative response in the liver that might be mediated by NF- $\kappa$ B activation (204, 211).

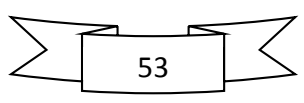


NO is a vasodilator and thus facilitates hepatic blood flow during reperfusion (222, 223). The positive effect of IPC on restoring hepatic microcirculation observed after 2 hours of reperfusion was demonstrated to not only disappear but even worsen when L-NAME, a competitive NOS inhibitor, was administered to rats before IPC $+\mathrm{I} / \mathrm{R}$ (183). Moreover, NO has been implicated in the damping effect of IPC on TNF- $\alpha$ release (213). The effect of preconditioning on eNOS expression has been studied, and eNOS expression increases with IPC relative to $\mathrm{I} / \mathrm{R}$ alone after 45 minutes of ischemia followed by 2 hours of reperfusion (168). In addition, the increased expression of eNOS was associated with higher plasma NOx. However, this study did not observe iNOS expression in IPC or the I/R group. On the other hand, iNOS gene expression is increased with IPC in living donor liver transplantation when the reperfusion period (before biopsies were obtained) is short (224).

The clinical effect of IPC has been studied in different settings. Studies on IPC before $\mathrm{I} / \mathrm{R}$ during liver resections can be categorized based on the type of ischemia applied. A number of studies focused on the effect of IPC in the setting of inflow occlusion; thus, studies can be further subdivided into those assessing continuous inflow occlusion and those studying intermittent inflow occlusion. However, only one study has been published on the effect of IPC before intermittent inflow obstruction (225). The other available studies compare continuous inflow obstruction with IPC + continuous inflow obstruction or IPC + continuous inflow obstruction with intermittent inflow obstruction alone (226-233). In addition, studies have assessed the effect of IPC combined with hepatic vascular exclusion that can be either total or selective and continuous or intermittent (234-237). Table 2 summarizes the published clinical studies involving IPC and liver resections.

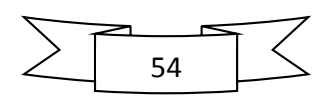




\begin{tabular}{|l|l|l|}
\hline Study & Vascular occlusion & Main results of IPC treatment \\
\hline Clavien et al. (226) & cPM & Reduced AST and ALT \\
\hline Clavien et al. (227) & cPM & Reduced AST and ALT \\
\hline Nuzzo et al. (230) & cPM & Reduced AST and ALT* \\
\hline Li et al. (233) & cPM & Reduced AST, ALT and bilirubin \\
\hline Chouker et al. (228) & cPM & Reduced PMN, AST and ALT \\
\hline Azoulay et a. (234) & SHVE & No difference \\
\hline Petrowsky et al. (231) & cPM + IPC $v s$. iPM - IPC & Equally effective \\
\hline Smyrniotis et al. (235) & iSHVE vs. IPC+cSHVE & $\begin{array}{l}\text { Equal for }<40 \text { min occl., increased } \\
\text { AST and caspase for }>40 \text { min occl. }\end{array}$ \\
\hline Heizmann et al. (229) & cPM & Reduced bleeding, fewer compl. \\
\hline Arkadopoulos et al. (236) & SHVE +/- IPC & Reduced AST and apoptosis \\
\hline Scatton et al. (225) & iPM +/- IPC & No difference \\
\hline Hahn et al. (232) & iPM vs IPC+cPM & Reduced AST and ALT** \\
\hline Jeon et al. (237) & THVE +/- IPC & No difference \\
\hline
\end{tabular}

Table 2. Clinical studies on the effectiveness of IPC. Only one of the studies reported a clinically significant difference when IPC was applied.

cPM = Continuous Pringle's maneuver

SHVE = Selective hepatic vascular exclusion

$I P C=$ Ischemic preconditioning

$i P M=$ Intermittent Pringle's maneuver

iSHVE = Intermittent selective hepatic vascular exclusion

cSHVE $=$ Continuous selective hepatic vascular exclusion

occl $=$ Occlusion

compl. $=$ Complications

THVE $=$ Total hepatic vascular exclusion

* Transection was performed during the IPC.

** Effect more pronounced in patients with cirrhosis.

Although some of the studies presented in table 2 report differences in laboratory values that suggest the protective effect of IPC compared with continuous inflow obstruction alone, the clinical effectiveness of this approach appears to be at best questionable, as indicated in a Cochrane review conducted in 2009 (238). The only study able to demonstrate a clinical difference reported significantly more bleeding and blood transfusions in the non-IPC group, making the findings somewhat difficult to interpret (229). In addition, intermittent vascular obstruction compares favorably with continuous vascular obstruction with and without IPC.

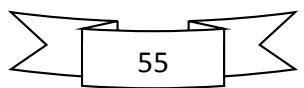


However, a notable gap in the literature regarding the possible protective effect of IPC when applied before intermittent vascular obstruction is evident (225).

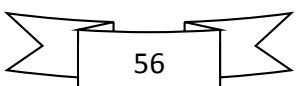




\section{Remote ischemic preconditioning (R-IPC)}

Hepatoduodenal ligament clamping to initiate IPC carries a small but significant risk of injury to the structures within the ligament. In addition, IPC is a time-consuming component of major liver resection, an operation that is often already long. Therefore, the idea of remote ischemic preconditioning (R-IPC), wherein another body part, such as a lower extremity, is rendered ischemic for a short period of time before the operation, is an appealing clinical concept. This concept was first published in the 1990s and has been primarily investigated in the heart $(239,240)$.

The first studies to investigate the possibility that R-IPC imparts protective effects in the setting of liver IRI were published in 2006 (241-243). With 3 cycles of 10 minutes of leg ischemia followed by 10 minutes of reperfusion before 25 minutes of total hepatic ischemia in rabbits, AST and ALT levels were significantly reduced at 2 hours of reperfusion compared with I/R alone (241). In addition, hepatic vein NOx was significantly increased in R-IPCtreated animals compared with those subjected to I/R alone. Hepatic blood flow at the end of reperfusion was also increased in R-IPC-treated animals. Taken together, the results indicated that R-IPC has a protective effect against hepatic IRI and that NO is involved in the early phase of reperfusion.

In a model involving 45 minutes of partial hepatic ischemia in rats preceded by three cycles of hind-limb preconditioning (10 minutes of ischemia and 10 minutes of reperfusion), serum ALT levels were significantly increased at 4 hours of reperfusion in the group subjected to $\mathrm{I} / \mathrm{R}$ alone (242). The main possible mechanism identified in the study was the induction of HO-1, as observed in hepatocytes after R-IPC.

R-IPC was applied as a single 10-minute occlusion of the femoral artery in rats followed by 15 minutes of reperfusion before either closure of the hepatic artery (branch) or total inflow occlusion of $70 \%$ of the liver (243). In this model, R-IPC reduced the IRI when applied before partial (artery only) hepatic ischemia but not when total circulation to the liver was occluded.

The role of R-IPC was further established in 2009 when a study involving 45 minutes of $70 \%$ hepatic ischemia followed by 3 hours of reperfusion reported that R-IPC (applied as 4

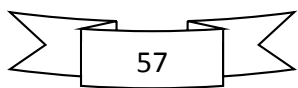


cycles of 5 minutes of ischemia and 5 minutes of reperfusion to the hind limb) increased red blood cell velocity and sinusoidal flow as well as decreased PMN adhesion (244).

In a mouse model, R-IPC applied as 10 minutes of ischemia followed by 10 minutes of reperfusion decreased IRI compared with I/R alone. Given that this effect was not observed in toll-like receptor 4 (TLR4)-mutant mice, it was concluded that functional TLR4 is required for R-IPC (245).

In a mouse model where R-IPC was administered as 6 cycles of 4 minutes of ischemia in the hind limb and 4 minutes of reperfusion before 40 minutes of $70 \%$ hepatic ischemia followed by 2 hours of reperfusion, R-IPC reduced the level of aminotransferases in plasma and preserved hepatic microcirculation (246). In the same model, R-IPC (with and without $\mathrm{I} / \mathrm{R}$ ) elevated NOx in the plasma. Furthermore, when the NO scavenger 2-(4.carboxyphenyl)4,4,5,5-tetramethylimiadozoline-1-ocyl-3-oxide potassium salt (C-PTIO) was administered before R-IPC, the protective effect disappeared (195). Further studies using this mouse model demonstrated that $\mathrm{eNOS}^{-/-}$mice are not protected by R-IPC and that eNOS protein expression is not increased with R-IPC compared with I/R alone in eNOS-wild type mice; thus, the protection is likely to be due to activation of the enzyme (247). To further evaluate the downstream mechanisms involved in the protective effects of NO in R-IPC, mice were administered 1H-(1,2,3)oxadiazole(4,3-a)quinoxalin-1-one (ODQ), an inhibitor of soluble guanylyl cyclase (sGC) (248). This inhibition resulted in levels of transaminases that did not significantly differ from those in I/R animals, whereas R-IPC without ODQ treatment offered the expected protection against IRI. Taken together, this series of studies provided evidence for the role of eNOS activation and subsequent NO production in the protective effects on the hepatic microcirculation via the cyclic guanosine monophosphate (cGMP) pathway during ischemia and early reperfusion.

The effects of R-IPC on the later phase of reperfusion have been less studied; however, 4 cycles of $5+5$ minutes (ischemia + reperfusion) of R-IPC to the hindlimb was shown in a rat model to result in greater sinusoidal diameter and reduced hepatocellular injury (measured as AST and ALT levels as well as signs of cell death upon histological examination) (249).

In one study, the effect of R-IPC on regeneration was assessed, and increased liver proliferation was shown 24 hours after R-IPC+R/I compared with I/R alone. Furthermore, IL6 mRNA was increased, whereas TNF- $\alpha$ expression was decreased, in the R-IPC group (250).

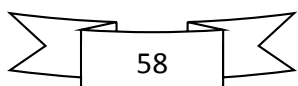


To date, no clinical studies exploring the usefulness of R-IPC in human liver surgery have been published. However, the preliminary results of 16 patients enrolled in a randomized study (www.clinicaltrials.gov NCT00796588) assessing the effects of R-IPC (3 cycles of 10 minutes of ischemia and 10 minutes of reperfusion to the lower limb) during major hepatectomy were presented at The Annual Meeting of the Society of Academic and Research Surgeons in January 2010 (251). In this report, the AST and ALT levels immediately after resection and 24 hours later were significantly reduced in the R-IPC group compared with the control group. Furthermore, the indocyanine green test (ICG-15) revealed enhanced liver function in the R-IPC group compared with controls immediately after resection (251). 
$\sum[60]$ 


\section{Microdialysis}

Bito introduced microdialysis $(\mu \mathrm{D})$ in 1966 as a method to investigate the concentration of electrolytes and amino acids in dog brain and subcutaneous tissue (252). In 1974, Urban Ungerstedt further developed the concept by applying a microdialysis membrane to a catheter, thus creating the first version of modern $\mu \mathrm{D}$ catheters (253).

The technique simulates the equilibrium between capillaries and interstitial fluid, which is based on the semi-permeable properties of the capillary wall. Small molecules can diffuse passively through this wall in a process that is driven by the concentration gradient of the substance and the oncotic pressure generated by larger molecules that do not pass through the wall.

The $\mu \mathrm{D}$ catheter is a double-lumen tube with a semi-permeable membrane at the tip. Through the outer lumen, an isotonic solution (perfusate) is continuously pumped, and at the tip, the solution comes into contact with the semi-permeable membrane that resembles capillary walls. The membrane has a pore size of 20 to $100 \mathrm{kDalton}(\mathrm{kDa})$ that can be selected based on what substance is measured. At the tip, the substance diffuses passively into the solution, which thereafter flows through the inner lumen of the catheter and is collected in a microvial (figure 11). The solution collected in the microvial (dialysate) can then be analyzed for the substances in question.

When presenting the results from $\mu \mathrm{D}$ studies, it must be kept in mind that the concentration in the fluid does not generally represent the actual concentration in the tissue but is a product of the recovery of the substance in the specific setting used during the sampling. When the recovery approaches $100 \%$, the concentration of the substance in the dialysate is close to the true concentration in the interstitial fluid. Given this limitation, $\mu \mathrm{D}$ studies are best performed as a comparison of concentrations from the same tissue/organ subjected to different situations or temporal change within the same organ.

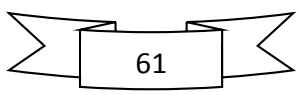




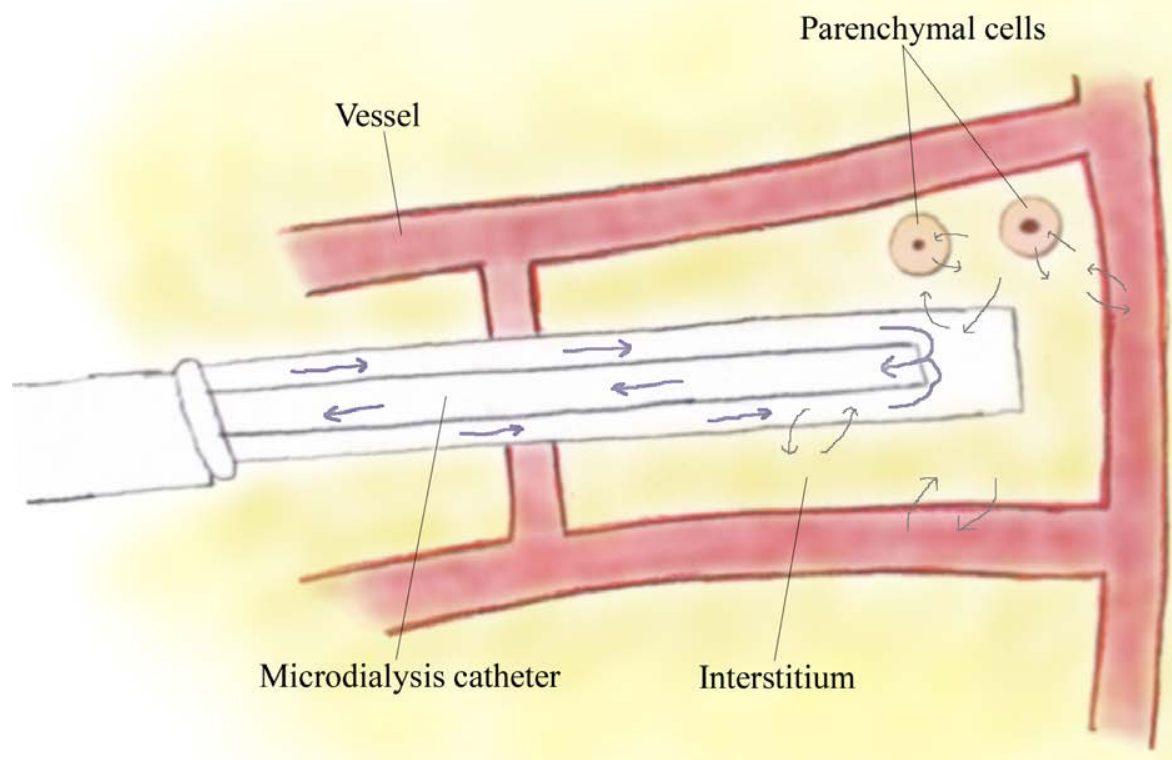

Figure 11. The principles of microdialysis. Perfusion fluid pumped through the catheter equilibrates with the interstitial fluid by diffusion over a semi-permeable membrane. The fluid is then sampled for analysis.

Many factors influence the recovery rate of substances within a microdialysis system. The membrane of the $\mu \mathrm{D}$ catheter is one of the most important factors that impacts recovery. Both the length of the membrane and the pore size determine the recovery rate. Thus, it is of great importance to choose catheters that allow for the diffusion of the substance to be analyzed. Another factor that can be controlled and standardized is the perfusion rate. At higher perfusion rates, the absolute recovery increases, but the dialysate becomes diluted, resulting in a net decrease in the recovery. Thus, lower perfusion velocities (typically 0.1-0.3 $\mu 1 / \mathrm{min})$ are desirable to increase the recovery rate. This necessity becomes somewhat problematic when short sampling intervals are needed to follow concentration changes over time because the low velocity combined with short sampling times make the recovered volumes small and difficult to analyze. Another factor that can be relatively easily controlled is the level of the microvial used to sample the dialysate in relation to the pump. If the microvial is positioned below the semi-permeable membrane, hydrostatic pressure at the tip of the catheter is increased, thus imposing the risk of excess water loss to the tissue from the perfusate. This loss increases the osmolality of the dialysate and yields a falsely elevated concentration of the substances to be analyzed. The sizes of the molecules in question are

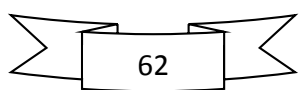


important given that larger molecules have less kinetic energy than smaller ones, resulting in less diffusion through the membrane. The kinetic energy is also affected by temperature; higher temperatures increase the kinetic energy, thus increasing the recovery. It has been estimated that a 1 degree Celsius increase in temperature increases the recovery by $1-2 \%$ (254).

In addition to the above- mentioned factors, it must be kept in mind that the introduction of microdialysis catheters is a traumatic process. Therefore, time must be allowed to pass before any reliable measurements can be performed. The time to reach equilibrium or "steady state" is typically set to 30 or 60 minutes; however, this time may be longer for some substances (255-257).

The microdialysis technique was introduced to clinical neurosurgery in the early $1990 \mathrm{~s}$ and has also been used in the setting of liver transplantation (258-261). In addition, the technique has been tested in the setting of liver resection (262). One of the advantages of this technique is that analysis can be performed "bedside" immediately after sampling, thus providing direct information about the organ in question.

In experimental settings, $\mu \mathrm{D}$ with an analysis of glucose, pyruvate, lactate and glycerol has been used in porcine models (263). An increase in glucose and lactate accompanied by decreased pyruvate during ischemia represents anaerobic metabolism, whereas increased glycerol is indicative of cell membrane disruption and thus tissue damage (120). 
$\sum[64]$ 


\section{Liver IRI study methodologies}

IRI is poorly defined and numerous obstacles are encountered when liver IRI is studied. First, the ethical aspects of performing such studies in human subjects must be considered. Although liver surgery has evolved dramatically from the high complication rates of the early days, there remains a large risk involved in this type of surgery. Thus, any unnecessary ischemic events are unethical, as is the prolongation of already long operations for research. These ethical concerns establish the boundary for clinical studies on liver IRI. To date, the trials published have largely relied on a combination of liver biopsies and blood sampling.

To gather more information on the nature of IRI, many animal models have been developed and used in various studies. The animal strains used ranges from small rodents (mice and rats) to larger mammals (dogs and pigs). Due to legislation, the use of dogs in clinical experiments has stopped in the Western world. The main motivation for the use of mammals, such as pigs, is that the anatomy and biology of these animals more closely resembles humans than rodents. On the other hand, rodents are more widely available for clinical research, and more commercially available reagents and kits can be used in the experiments, thus making the results more reliable.

Irrespective of what species is used, the study of IRI mechanisms is complicated as the cascade is multifactorial and interactions between different signaling molecules and cytokines may change over time. The early models of total liver ischemia posed the problem of blood stagnation in the bowel. This problem was overcome by non-physiological shunts that were cumbersome and might have affected the results (264). Today, most animal models of liver IRI rely on partial or segmental liver ischemia, circumventing this problem. However, this approach makes it difficult to know how much ischemia is applied, and no reliable method is available to measure or grade the ischemia. A possible method for investigating the different effects of various grades of ischemia in addition to the time factor is the use of selective occlusion of the hepatic artery compared with PM (243).

The sampling has largely been based on repeated biopsies from the liver and organ harvesting as well as blood sampling. Thus, the chain of events and temporal changes have been difficult to follow in one or a few animals, and many animals have been sacrificed to reveal causal relationships. This experimental design, however, is complicated by the fact that IRI is poorly defined and by the fact that various methods are used to measure the

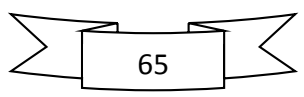


phenomenon. The undisputed basic biochemical markers include the liver transaminases and ultimately the histological signs of tissue injury. Although the assessment of histology can be considered a subjective art, some objective guidelines can be used. In addition to the factors included in the score used in our studies, the infiltration of PMNs is commonly used to describe histological signs of IRI. The dimensions of the score used in the present studies (sinusoidal congestion, cytoplasmic vacuolation, liver necrosis) are the same as those used in another widely used system, although the grading is somewhat different (265). Apoptosis is an important component of IRI that is difficult to assess with basic pathology staining, and the signs of necrosis evolve over time; therefore, the length of the experiment influences the findings. The appearance of PMNs is also a time-dependent (see chapter 7) sign of inflammation that is not captured with the scoring system that is commonly employed.

In this thesis, a known model of rat liver IRI was modified with the addition of $\mu \mathrm{D}$ to gather more information about the local metabolism and temporal changes in the liver (266). In study II, $\mu \mathrm{D}$ was added to clinical praxis along with a short period of IPC. 


\section{Aims of the thesis}

The overall aim of this thesis was to investigate methods to reduce IRI in the liver.

Specific aims for studies I-IV:

I To compare the effectiveness of IPC and R-IPC before IRI in a rat model and to investigate whether commercially available kits for microdialysis could be used to monitor liver IRI.

II To investigate whether IPC before the interrupted PM exhibits clinical benefits compared with the interrupted PM alone in a randomized controlled trial.

III To compare the involvement of NO and iNOS in the effects of IPC and R-IPC.

IV To investigate whether nitrite administered before ischemia reduces IRI and the activation of iNOS transcription. 
$\sum[68]$ 


\section{Material and methods}

\section{Ethical}

The study protocols for study I (2-09), study III (2-09) and study IV (56-12) were approved by the regional ethics committee for animal experiments, Linköping, Sweden. The study protocol for study II (M100-06) was approved by the regional ethics board, Linköping, Sweden.

\section{Animals (study I, III and IV)}

Male Sprague-Dawley rats (258-444 g) were used in studies I, III and IV.

Before the experiments began, the animals were acclimatized for one week in the laboratory pet house. The rats had free access to standard rat food pellets and tap water in a 12 -hour light/dark environment at $21^{\circ} \mathrm{C}$.

\section{Patients (study II)}

Patients with planned resections of at least two liver segments due to metastatic liver disease or the suspicion of gallbladder cancer (T1-2, tumors confined to the gallbladder wall) were asked to participate in the study. Patients with intended portal venous ligation, multi-organ resection or suspected or proven chronic liver disease were not included in the study.

If no differences were observed in 16 patients with IPC, any difference missed due to low power would be of minimal clinical interest.

The patients were stratified according to the size of the intended resection and randomized to either preconditioning or no preconditioning prior to the PM (figure 12).

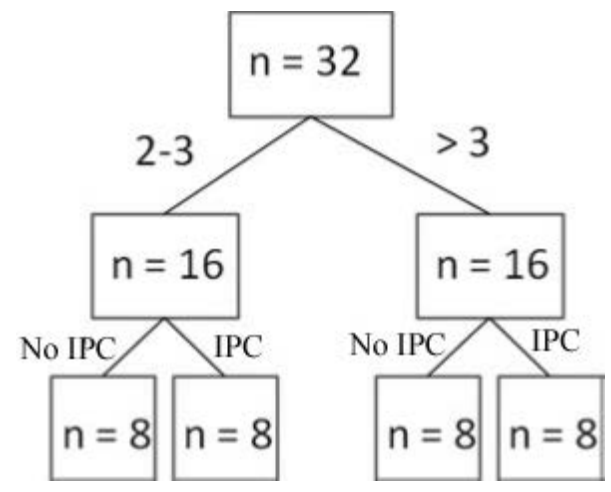

Figure 12. Randomization and stratification (study II). Patients were stratified according to the number of segments resected and randomized within each stratum to IPC or no IPC prior to the $P M$.

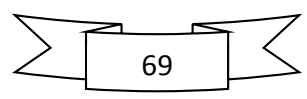




\section{Anesthesia and surgery}

\section{Animals}

The rats (studies I, III and IV) were anesthetized with isoflurane, and $0.05 \mathrm{mg} / \mathrm{kg}$ buprenorphine was administered s.c. for pain relief. The animals were then intubated with a 16-G intravenous catheter and ventilated throughout the experiment. During the experiment, the animals were monitored, and body temperature was maintained within $38-39^{\circ} \mathrm{C}$. Laparotomy was performed via a midline incision, and the ligament attachments of the liver were divided. Directly after intubation and every hour throughout the experiment, the animals received 5-ml warm Ringer's acetate s.c.

\section{Patients}

All patients (study II) received anesthesia according to the low CVP concept with a target CVP $\leq 5 \mathrm{mmHg}$. Fluid restriction and nitroglycerine were used according to clinical practice. The operations were performed through a right subcostal incision angled and extended to the sternum.

After exploration without findings contraindicating resection, patients were stratified (23 or $>3$ segments) and then randomized to either IPC or no IPC. PM was applied with cotton tape around the hepatoduodenal ligament. The intermittent PM was performed with 15-minute closure of the hepatoduodenal ligament and 5-minute open circulation for 1 to 3 cycles.

The IPC group underwent 10-minute closure of the ligament followed by 10-minute open circulation (IPC) administered prior to the intermittent PM.

Parenchymal transection was achieved with the CUSA, and liver biopsies were obtained from microscopically healthy portions of the liver at the end of the operation.

\section{IPC, R-IPC and liver ischemia (studies I, III and IV)}

In the IPC group, a clamp was applied over the vascular pedicle to the left lateral lobe and then removed after 10 minutes, allowing for 10-minute reperfusion before the ischemic assault.

Remote IPC was induced by placing a tight tourniquet around the right hind leg for 10 minutes followed by 10 minutes of reperfusion.

The ischemia lasted 60 minutes (study I and III) and was followed by 4 hours of reperfusion. In study IV, no preconditioning was applied, and the ischemia was reduced to 45 minutes while maintaining 4 hours of reperfusion.

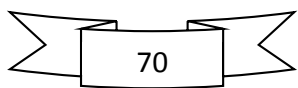




\section{Administration of nitrite (study IV)}

Two minutes before the application of ischemia, $480 \mathrm{nM}$ sodium nitrite dissolved in $\mathrm{NaCl}$ $(0,24 \mathrm{ml})$ was administered directly into the caval vein using a $30-\mathrm{G}$ needle.

\section{Local metabolism (microdialysis)}

\section{Animals}

The model of segmental ischemia in the left lateral lobe has been previously established (266). In studies I, III and IV, microdialysis was added to this model. A CMA 20 Elite microdialysis probe was inserted in both the left lateral and right lateral (control) liver lobes and perfused at a rate of $1.0 \mu \mathrm{l} / \mathrm{min}$ with perfusion fluid $\mathrm{T} 1(\mathrm{NaCl} 147 \mathrm{mmol} / \mathrm{L}, \mathrm{KCl} 4$ $\mathrm{mmol} / \mathrm{L} \mathrm{CaCl} 22.3 \mathrm{mmol} / \mathrm{L}$ ). Before insertion, the probes were pre-perfused. After probe insertion, 35 minutes were allowed to pass to achieve steady state, and 20 minutes of baseline sampling of the microdialysate was performed before ischemia was induced. From the beginning of ischemia and throughout the reperfusion period, the microdialysate was sampled. Glucose, glycerol, lactate and pyruvate were instantly analyzed with the clinical bedside analyzer ISCUS (CMA Microdialysis).

\section{Patients}

In study II, 63 microdialysis CMA catheters were used. Two catheters were inserted into the FLR and attached to the Glisson capsule using 4/0 absorbable sutures. The $\mu \mathrm{D}$ catheters were fastened to the skin with tape and connected to a 107$\mu \mathrm{D}$ CMA pump that the patients kept in a waist belt during the post-operative period (figure 13).

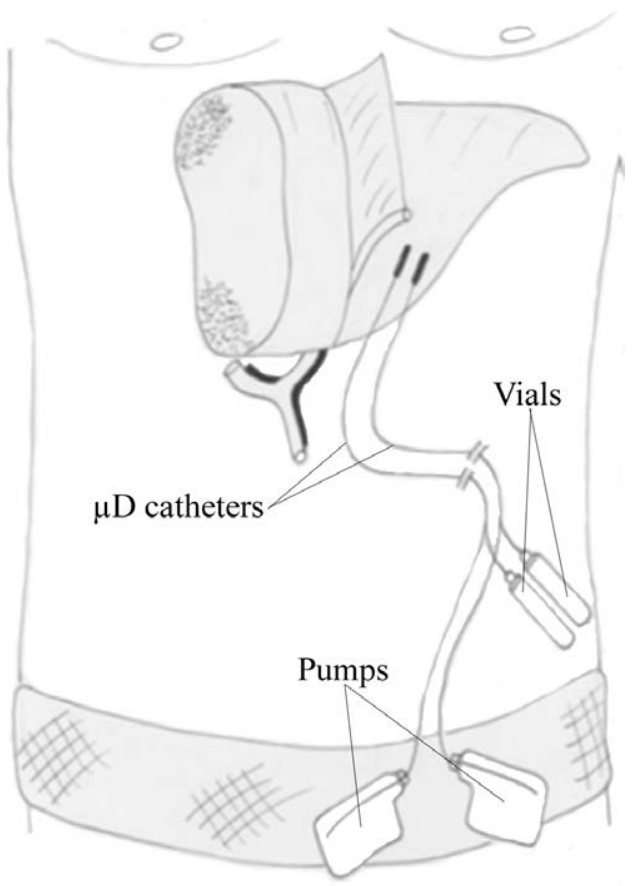

Figure 13. Microdialysis in a clinical setting. Perfusion fluid is pumped through the catheters and sampled in the vials.

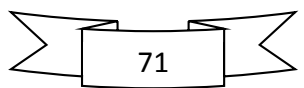


The catheters were perfused with Ringers acetate at a rate of $2.0 \mu 1 / \mathrm{min}$ during surgery at the surgical recovery department. Initially, 20 minutes were allowed to achieve a steady state; subsequently, samples were collected every $1 / 2$ hour during surgery and every hour at the recovery department.

When the patient was transferred to the surgical ward, the perfusion velocity was changed to $0.3 \mu \mathrm{l} / \mathrm{min}$, and sampling was performed every $4^{\text {th }}$ hour until the evening of postoperative day (POD) 4 when the catheters were removed.

The dialysate from one of the sampled microvials was immediately analyzed using the bedside analyzing equipment ISCUS, whereas the other microvial was frozen at $-20^{\circ} \mathrm{C}$ for later analysis of NOx.

\section{General}

In all of the studies, the automatic bedside analysis machine ISCUS was used for immediate analysis of glucose, pyruvate, lactate and glycerol. The ISCUS machine uses a single-beam filter photometer to detect light emitted at a wavelength of $530 \mathrm{~nm}$. All of the enzymatically catalyzed reactions lead to the formation of quinoneimine at a rate that is proportional to the substance to be measured (information from CMA Microdialysis).

Glucose concentrations are measured as follows:

D-Glucose $+\mathrm{O}_{2} \rightarrow$ gluconolactone $+\mathrm{H}_{2} \mathrm{O}_{2}$

(catalyzed by glucose oxidase)

$2 \mathrm{H}_{2} \mathrm{O}_{2}+$ phenol +4 -amino-antipyrine $\rightarrow$ quinoneimine $+4 \mathrm{H}_{2} \mathrm{O}$

(catalyzed by peroxidase)

Detection range: $0.1-25 \mathrm{mM}$, deviation $<5 \%$.

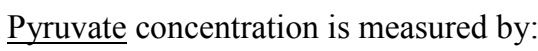

Pyruvate $+\mathrm{P}_{\mathrm{i}}+\mathrm{O}_{2} \rightarrow$ acetylphosphate $+\mathrm{CO}_{2}+\mathrm{H}_{2} \mathrm{O}_{2}$

(catalyzed by pyruvate oxidase)

$2 \mathrm{H}_{2} \mathrm{O}_{2}+$ TOOS +4 -amino-antipyrine $\rightarrow$ quinoneimine $+4 \mathrm{H}_{2} \mathrm{O}$

(catalyzed by peroxidase)

TOOS = N-ethyl-N-(2-hydroxy-3-sulfopropyl)-m-toluidine

Detection range: $10-1500 \mu \mathrm{M}$, deviation $<5 \%$.

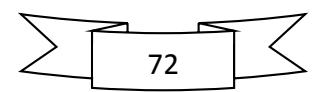


Lactate is measured by:

L-Lactate $+\mathrm{O}_{2} \rightarrow$ pyruvate $+\mathrm{H}_{2} \mathrm{O}_{2}$

(catalyzed by lactate oxidase)

$\mathrm{H}_{2} \mathrm{O}_{2}+4$-chloro-phenol + 4-amino-antipyrine $\rightarrow$ quinoneimine $+2 \mathrm{H}_{2} \mathrm{O}+\mathrm{HCl}$

(catalyzed by peroxidase)

Detection range: $0.1-12 \mathrm{mM}$, deviation $<5 \%$.

Glycerol concentration is obtained by:

Glycerol + ATP $\rightarrow$ glycerol-3-phosphate + ADP

(catalyzed by glycerol kinase)

glycerol-3-phosphate $+\mathrm{O}_{2} \rightarrow$ dihydroxyacetone phosphate $+\mathrm{H}_{2} \mathrm{O}_{2}$

(catalyzed by glycerol-3-phosphate oxidase)

$\mathrm{H}_{2} \mathrm{O}_{2}+$ DCHBS +4 -amino-antipyrine $\rightarrow$ quinoneimine $+2 \mathrm{H}_{2} \mathrm{O}+\mathrm{HCl}$

(catalyzed by peroxidase)

DCHBS $=3,5$-dichloro-2-hydroxy-benzene sulfonic acid

Detection range: $0.01-1.5 \mathrm{mM}$, deviation $<5 \%$.

\section{Blood sampling and analysis}

Animals

In studies I and III, blood was sampled in a 2-ml syringe both at the beginning and the end of the experiment from the exposed external jugular vein. In study IV, blood was only sampled immediately before the animals were exsanguinated, as previous studies have demonstrated only small variations at baseline. The blood was centrifuged at $3000 \mathrm{rpm}$ for 7 minutes in LH tubes.

AST and ALT were analyzed according to laboratory standards (Department of Clinical Chemistry, University Hospital, Linköping, Sweden). Other analyses are described below.

\section{Patients}

Blood sampling in study II for Hb, WBC, albumin, AST, ALT, bilirubin, INR and lactate was performed preoperatively, directly after PM, at the end of surgery, at $8 \mathrm{pm}$ on the day of surgery and on POD 1-4 at 7 am. All measurements were performed according to laboratory standards at the Department of Clinical Chemistry, University Hospital, Linköpiong, Sweden. In addition, capillary glucose levels were measured every $4^{\text {th }}$ hour. 


\section{Tissue sampling}

\section{Animals}

Directly after the final blood sampling, the liver was harvested. For study I, the tissue was preserved in formalin for histological analysis; for study III, the tissue was preserved in liquid nitrogen. In study IV, both methods were used. In study IV, liver biopsies were also obtained after 15 minutes of ischemia and after 40 minutes of ischemia; this tissue was stored immediately in liquid nitrogen.

The tissue sampled for histology was fixed in formalin (4\%) overnight and embedded in Technovit 8100-plastic. Liver sections $(2 \mu \mathrm{m})$ were hematoxylin-eosin $(\mathrm{H} \& \mathrm{E})$ stained and coded before examination by a pathologist blind to the experimental design (studies I and IV). In study I, samples from 3 randomly chosen animals in each group were examined, whereas samples from all the animals were examined in study IV.

The degree of liver injury was estimated using the scoring system described by Calabrese et al. (267). The signs noted are as follows: sinusoidal congestion, cytoplasmic vacuolation, and liver necrosis. Sinusoidal congestion was scored as $0-4$, where $0=$ none, $1=$ less than $10 \%$ hepatic tissue, $2=10-40 \%, 3=40-70 \%$ and $4=$ greater than $70 \%$. Cytoplasmic vacuolation was scored as $0-4$ : $0=$ none, $1=$ less than $10 \%, 2=10-40 \%, 3=40-70 \%$ and $4=$ more than $70 \%$ of hepatic tissue. Liver necrosis was scored as $0-2$, where $0=$ none, $1=$ less than half of the hepatocytes and $2=$ more than half of the hepatocytes.

Tissue preserved in liquid nitrogen was analyzed for iNOS and IL-1 receptor mRNA (study III) and iNOS mRNA (study IV) as described below.

\section{Patients}

The liver biopsies were immediately submerged in liquid nitrogen and later freeze-dried. Glycogen in the liver tissue was quantified with the BioVision Glycogen Assay kit (BioVision Research Products, Mountain View, CA, USA). Freeze-dried tissue was placed in ice-cold Eppendorf tubes, crushed with mortar and pestle and extracted into sterile water. Enzymes were then inactivated by boiling for $5 \mathrm{~min}$. Subsequently, the tubes were centrifuged at 13,000 rpm for $5 \mathrm{~min}$. The supernatants were added to 96-well plates. As recommended in the assay kit, the glucose levels in the extracts (corresponding to 500-1000 mg freeze-dried tissues) were measured with and without hydrolyzing the glycogen by glucoamylase. After the reaction with OxiRed, the absorbance was finally colorimetrically measured in an enzyme-linked immunoassay (ELISA) reader at $550 \mathrm{~nm}$. The glycogen level was calculated

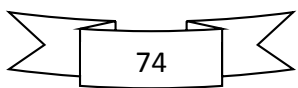


using the standard curve included in the kit, and the result is reported as $\mu \mathrm{g}$ glycogen per mg freeze-dried tissue.

\section{NOx (studies II, III and IV)}

In the serum and microdialysate, the sum of $\mathrm{NO}_{2}{ }^{-}$and $\mathrm{NO}_{3}{ }^{-}$were analyzed following the instructions in the commercial "Nitrite/Nitrate Fluorometric Assay Kit". Prior to analysis, the serum was ultrafiltrated through a $10-\mathrm{kDalton}$ cut-off filter. The microdialysate was directly analyzed. Standard curves were plotted. For nitrate, $10 \mu \mathrm{L}$ of sample was diluted in $70 \mu \mathrm{L}$ assay buffer. Aliquots of $10 \mu \mathrm{L}$ of enzyme cofactor and $10 \mu \mathrm{L}$ of nitrate reductase mixture were added to the buffered sample. After 30 minutes of incubation at room temperature, 10 $\mu \mathrm{L}$ of DAN (2,3-diaminonaphthalene) was added and incubated for another 10 minutes. For nitrite, $10 \mu \mathrm{L}$ of sample was diluted with $90 \mu \mathrm{L}$ assay buffer, and $10 \mu \mathrm{L}$ of DAN was added. Both nitrate and nitrite samples were then incubated for 10 minutes, and $20 \mu \mathrm{L}$ of $\mathrm{NaOH}$ was added. All samples were read in triplicate with fluorometry using an excitation wavelength of $355 \mathrm{~nm}$ and emission wavelength of 430-460 $\mathrm{nm}$.

\section{iNOS mRNA in liver tissue (studies III and IV)}

Approximately $10 \mathrm{mg}$ of dry weight liver tissue was disrupted and homogenized in a MicroDismembrator at $2900 \mathrm{rpm}$ for $30 \mathrm{sec}$. The RNA extraction was performed according to the manufacturer's protocol (Qiagen, Valencia CA, USA) with the modification of eliminating $\beta$ mercaptoethanol. The RNA was eluted in $300 \mu \mathrm{L}$ of RNAse-free water, and the RNA concentration and purity $(\sim 2,0)$ were measured at $260 \mathrm{~nm}$ and $260 / 280 \mathrm{~nm}$, respectively. RNA was stored at $-70^{\circ} \mathrm{C}$ until assayed. Approximately $0.5 \mu \mathrm{g}$ RNA was used in the reverse transcriptase cDNA conversion with High Capacity cDNA Reverse Transcription Kit in a total volume of $20 \mu \mathrm{L}$ according to manufacturer's protocol (Applied Biosystems, CA, USA). cDNA samples were stored at $-20^{\circ} \mathrm{C}$ until assayed. For the real-time PCR reaction, $2 \mu \mathrm{L}$ of sample containing $50 \mathrm{ng}$ cDNA $(0,025 \mu \mathrm{g} / \mu \mathrm{L})$ was used in a total reaction volume of $20 \mu \mathrm{L}$. Samples were analyzed in triplicate with the Fast Master Mix and TaqMan Gene Expression Assay in a 7500 Fast Instrument. For results, the mean $\mathrm{Ct}$ values were normalized against the mean $\mathrm{Ct}$ for GAPDH (Glyceraldehyde-3-phosphate dehydrogenase) and are presented as $\Delta \mathrm{Ct}$.

\section{IL-1R mRNA in liver tissue (study III)}

Quantitative PCR for IL1R was performed with the Fast SYBR Green Master Mix. The final concentration of the primers was $100 \mathrm{nM}$. In the negative controls, cDNA was replaced by

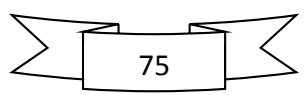


distilled water. The final amount of cDNA in each reaction was comparable to $1 \mathrm{ng}$ of RNA. The PCR program was set for 40 cycles of $95^{\circ} \mathrm{C}$ for $20 \mathrm{sec}, 95^{\circ} \mathrm{C}$ for $1 \mathrm{sec}$ and $60^{\circ} \mathrm{C}$ for $20 \mathrm{sec}$. At the end of the reaction, a dissociation curve analysis was performed. Specific primers for rat IL-1R were used. GAPDH and beta-actin (b-actin) were utilized as housekeeping genes. The primers were designed using Primer Express. All reactions, including no-template controls and endogenous control probes, were performed by the epMotion pipetting robot in duplicate. The results were analyzed using the $\Delta \mathrm{Ct}$ method and are presented as relative gene expression. The 7900 Fast Real-Time PCR system with 7900 System SDS 2.3 Software was used according to the manufacturer's protocol.

\section{Statistical methods}

\section{Animals}

Data are presented as the means (SEMs) unless otherwise stated. A $p$-value $<0.05$ was considered statistically significant for all statistical calculations. ANOVA followed by posthoc analysis with Fisher's LSD method was used to compare multiple groups in study I. Temporal changes within the groups were analyzed using the t-test or the Mann-Whitney U test. Statistica 8.0 software (StatSoft Inc., Tulsa, Oklahoma, USA) was used for all statistical calculations.

\section{Patients}

Patients with IPC $(n=16)$ were compared with the controls $(n=16)$. The patients with IPC (n $=8)$ were then compared with the controls $(n=8)$ within the same stratum $(2-3$ segments and

$>3$ segments resected). The results are presented for these stratified groups unless otherwise indicated in the text.

A secondary comparison was made between minor (2-3 segments, $\mathrm{n}=16)$ and major ( $>3$ segments, $n=16$ ) resections to control for confounding changes depending on resection size. The $\mu \mathrm{D}$ samples were grouped into the following 7 different phases: baseline (sample 1), preoperative (samples 2-7), post-operative on the day of surgery (samples 8-24), POD 1 (samples 25-30), POD 2 (samples 31-36), POD 3 (samples 37-42) and POD 4 (samples 4346). Individuals were nested in the IPC and control groups and assigned as the random factor in an ANOVA. A Tukey post-hoc analysis was performed if significant differences were observed between the groups in the ANOVA. Non-parametric values are presented as medians (ranges) and were analyzed with the Mann-Whitney U test; parametric values are presented as the means (SEMs) and were analyzed using Student's t-test. STASTICA 8.0

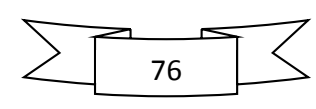


software (StatSoft; Tulsa, OK, USA) was used for all statistical calculations. A $p$-value of $<$ 0.05 was considered statistically significant.

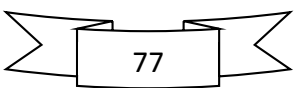




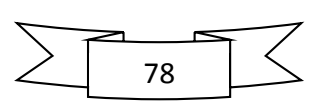




\section{Results}

\section{Study I}

Blood analyses

Serum AST levels were increased in the IRI group (155 (20.9) IU/L) compared with the IPC group (71.5 (19.6) IU/L) or in the R-IPC group (96.6 (12.4) IU/L) at 4 hours of reperfusion. The differences were significant with $p$-values of 0.004 and 0.04 , respectively. However, the difference between the IPC and R-IPC groups was not significant (figure 14).

After 4 hours of reperfusion, serum ALT levels were significantly increased in the IRI group (107.4 (15.5) IU/L) compared with the IPC group (41.6 (11.3) IU/L) $(p=0.003)$. The levels in the R-IPC group (68.1 (14.9) IU/L) did not differ significantly from the IRI group ( $p$ $=0.064$ ) or the IPC group (figure 15). In the sham group, only a small increase in the AST and ALT levels was observed during the reperfusion.

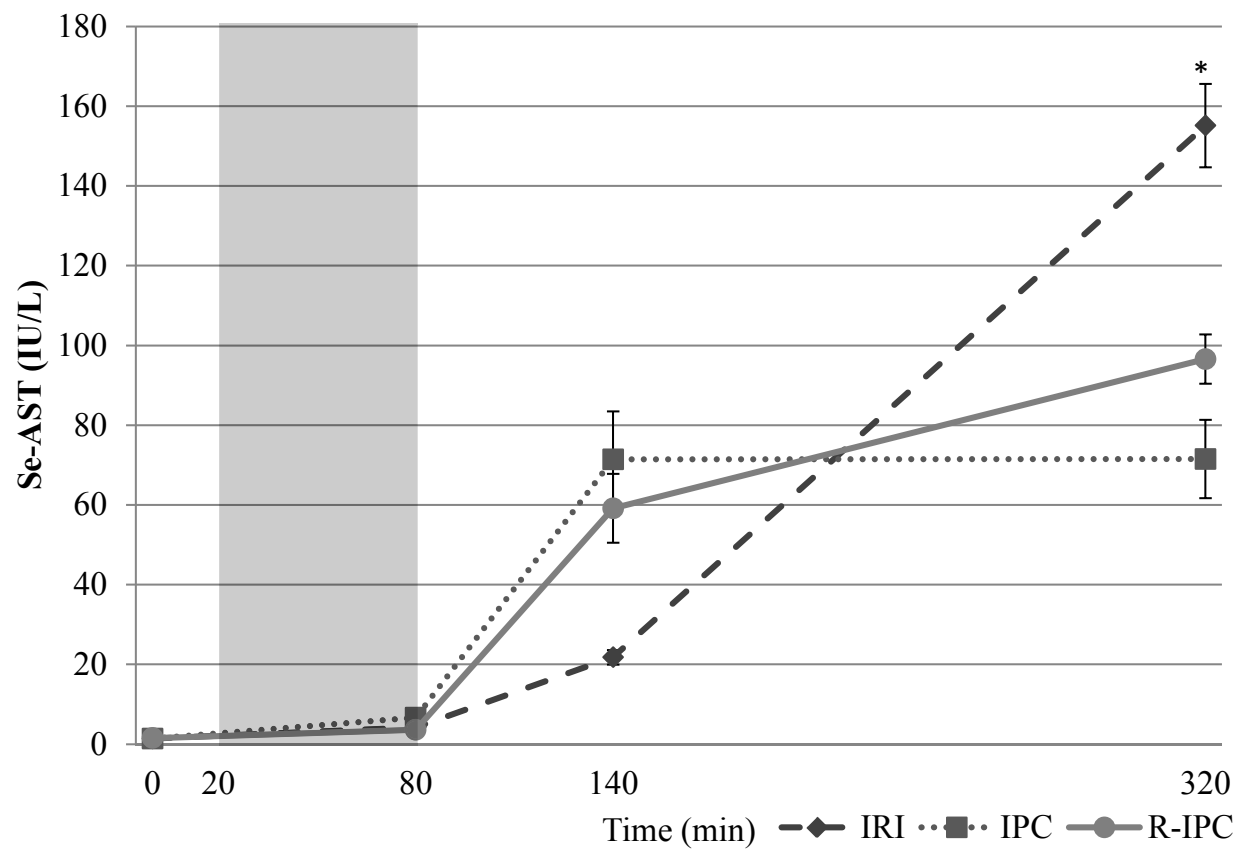

Figure 14. Serum AST levels (study I). Mean \pm SEM serum AST levels after 1-hour segmental liver ischemia followed by 4 hours of reperfusion in rats pre-treated with IPC or R-IPC compared with non-treated rats (IRI). The shaded area represents the ischemic phase. $* p=0.004$ (IRI vs. IPC) and $p=0.04$ (IRI vs. R-IPC).

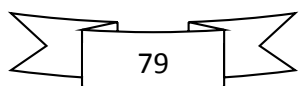




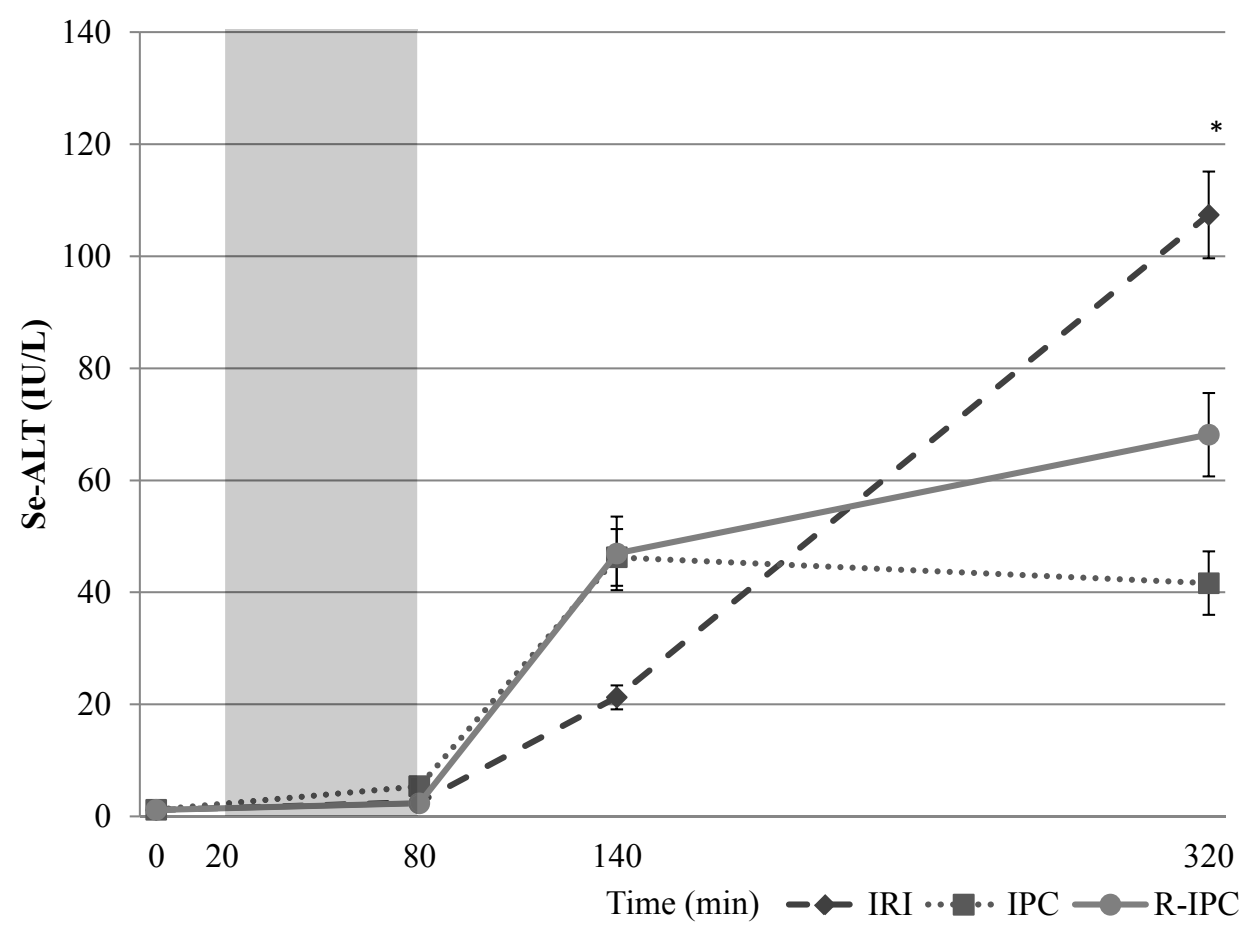

Figure 15. Serum ALT levels (study I). Mean \pm SEM serum ALT levels after 1-hour segmental liver ischemia followed by 4-hour reperfusion in rats pre-treated with IPC or R-IPC compared with non-treated rats (IRI). The shaded area represents the ischemic phase.

$* p=0.003$ (IRI vs. IPC).

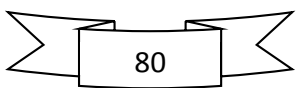




\section{Local metabolism measured by microdialysis}

\section{Glucose}

Glucose increased approximately 2-fold in the ischemic lobe in the IRI group compared with the control lobe during ischemia. In both the IPC and the R-IPC groups, similar patterns were noted, although the overall changes were of a reduced magnitude.

In the IPC group, glucose levels increased significantly to $10.2(0.7) \mathrm{mM}$ in the ischemic lobe after preconditioning $(\mathrm{t}=20)$ compared with $7.2(0.4) \mathrm{mM}$ at baseline $(\mathrm{t}=0, p$ $=0.0002$ ) (figure 16). This early increase in parenchymal glucose was not observed in the IRI or the R-IPC groups. At $\mathrm{t}=20$, glucose levels were significantly increased in the ischemic lobes in the IPC group (10.2 (0.7) $\mathrm{mM})$ compared with the corresponding lobes in the IRI group (7.5 $(0.6) \mathrm{mM})(p=0.006)$ and with the ischemic lobe in the R-IPC group (7.0 (0.6) $\mathrm{mM})(p=0.001)$. On the other hand, the rise in glucose during ischemia was less pronounced in the IPC (7.1 (1.2) $\mathrm{mM})$ group than in the IRI (12.7 (1.6) $\mathrm{mM})$ group. At $\mathrm{t}=110$, this difference achieved statistical significance $(p=0.005)$. In the R-IPC (10.3 (1.1) mM) group, a trend towards increased glucose levels was observed compared with the IPC group, although the difference was not statistically significant $(p=0.091)$.

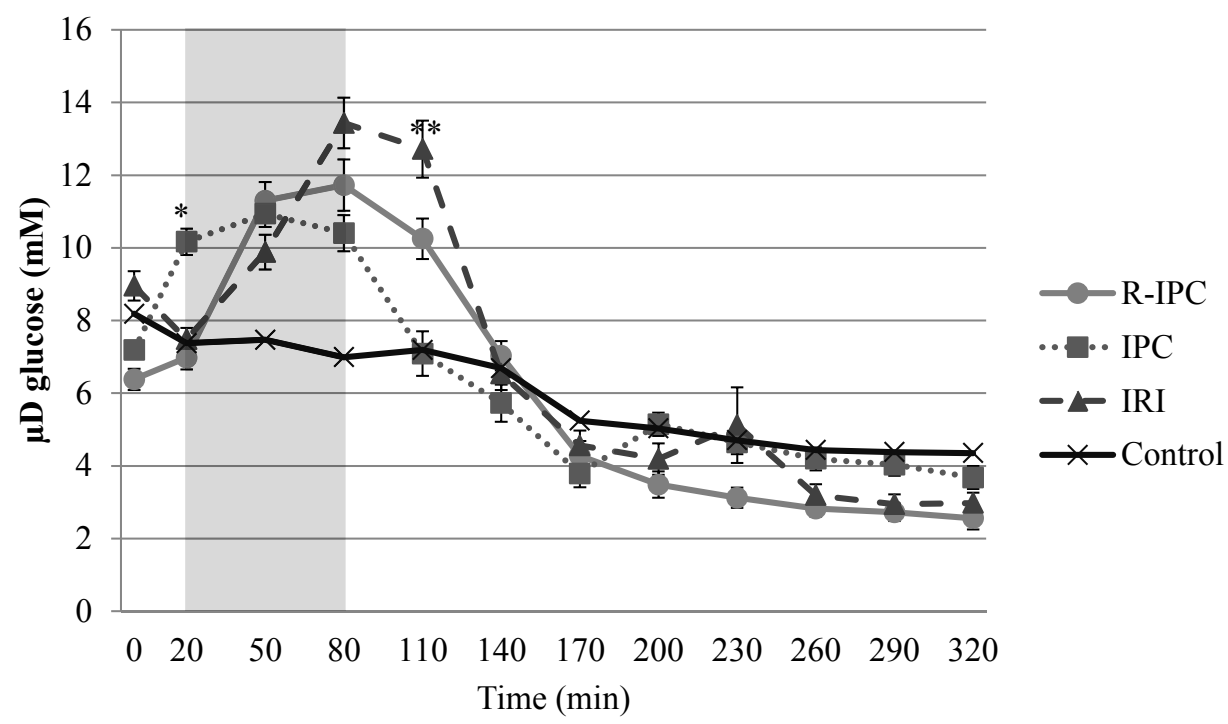

Figure 16. $\mu D$ glucose (study I). Mean $\pm S E M \mu D$ glucose in the ischemic liver segment after 1-hour segmental liver ischemia and 4-hour reperfusion in rats pre-treated with IPC or RIPC compared with non-treated rats (IRI) and the combined results from the control (nonischemic) segments (control). The shaded area represents the ischemic phase.

$* p=0.006$ (IRI vs. IPC) and $p=0.001$ (IPC vs. R-IPC).

$* * p=0.005$ (IRI vs. IPC).

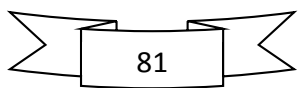




\section{Pyruvate}

In the ischemic segment in the IRI group, pyruvate levels were reduced to approximately $20 \%$ of the level in the control segment at the end of ischemia. The corresponding values in the IPC and R-IPC groups were $65 \%$ and $40 \%$, respectively. Given the large interindividual variations in the pyruvate levels, no significant differences were noted between the groups (figure 17).

In the sham group, no significant differences were noted between the two lobes in the microdialysate (data not shown).

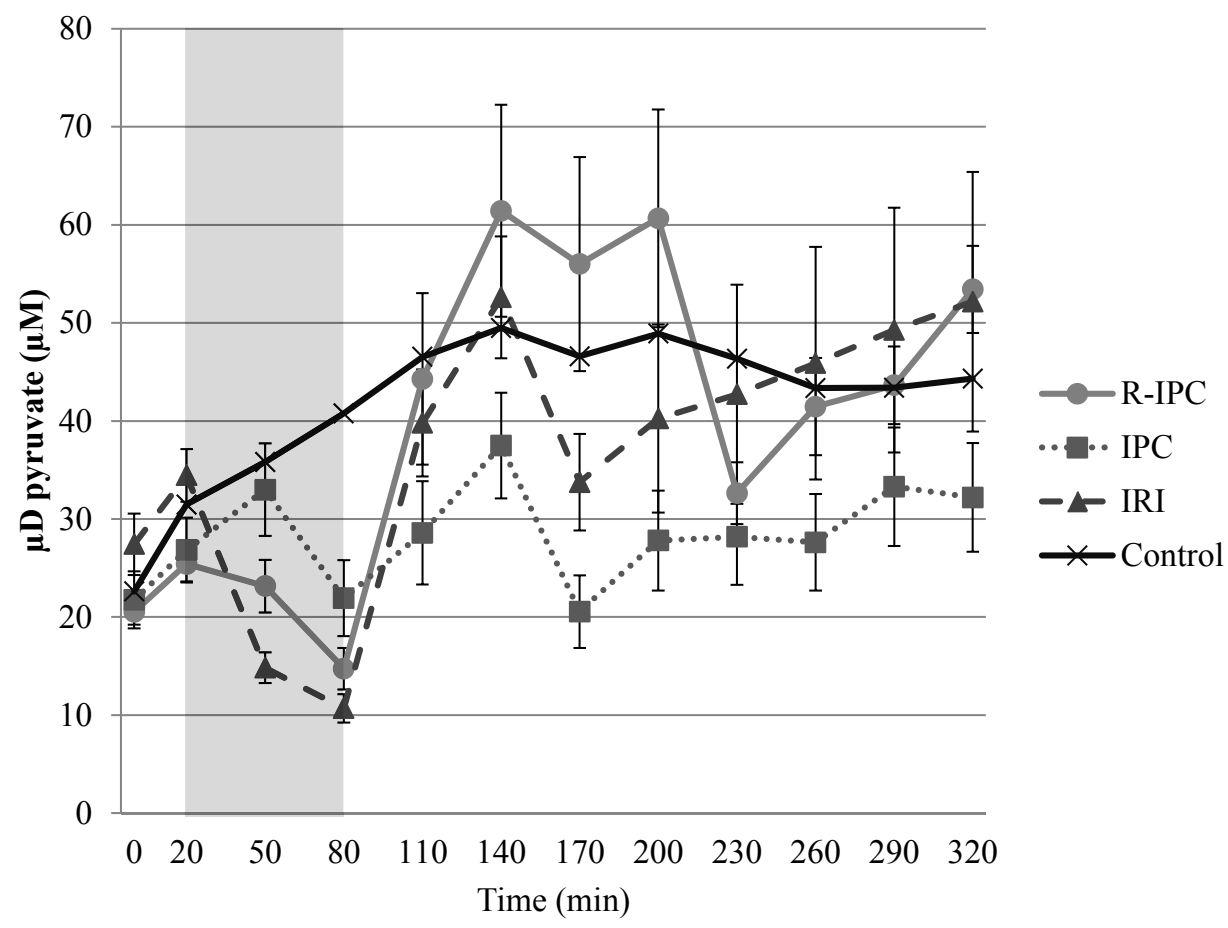

Figure 17. $\mu D$ pyruvate (study I). Mean \pm SEM $\mu D$ pyruvate in the ischemic liver segment during 1-hour segmental liver ischemia and 4-hour reperfusion in rats pre-treated with IPC or R-IPC compared with non-treated rats (IRI) and the combined results from the control (non-ischemic) segments (control). The shaded area represents the ischemic phase.

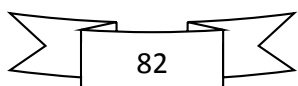




\section{Lactate}

In ischemic lobes in the IRI group, the lactate levels increased 3 fold during the ischemic phase compared with the control lobe. The same pattern was observed in the other groups. In the IPC group, increased lactate was observed in the ischemic segment during preconditioning $(t=20)$. This increase was 2.5-fold higher than the initial value $(t=0$; from $0.92(0.08)$ to 2.5 $(0.2) \mathrm{mM})(p<0.001)$. This change was not observed in the IRI or the R-IPC groups. The differences between the IPC group compared with the IRI and R-IPC groups $(2.5(0.2) \mathrm{mM}$ vs. $1.3(0.2)$ and $(1.8(0.4) \mathrm{mM})$ at $\mathrm{t}=20$ were statistically significant $(p=0.002$ and $p=$ 0.045 , respectively). Otherwise, no differences were observed between the groups (Figure $18)$.

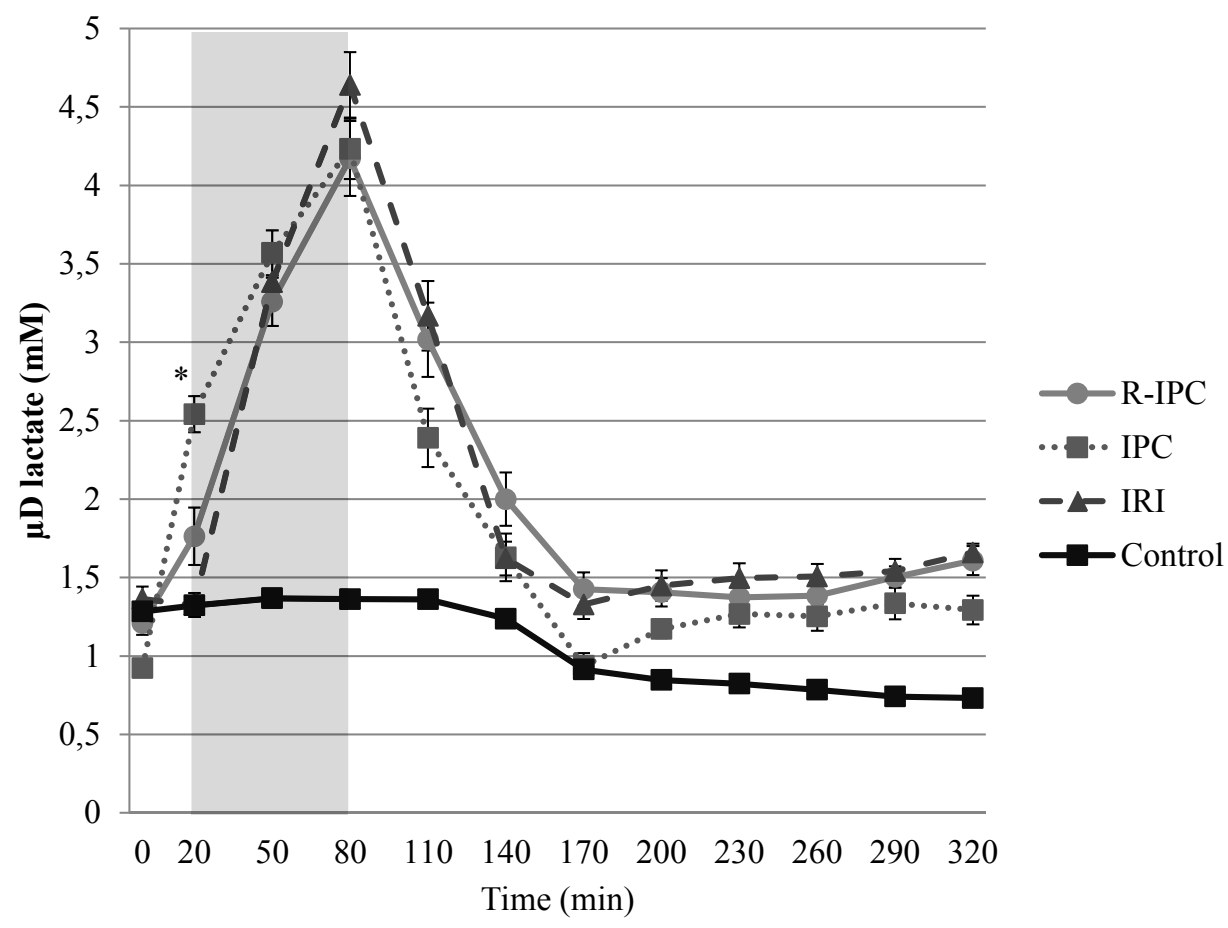

Figure 18. $\mu D$ lactate (study I). Mean $\pm S E M \mu D$ lactate in the ischemic liver segment during 1-hour segmental liver ischemia and 4-hour reperfusion in rats pre-treated with IPC or $R$ IPC compared with non-treated rats (IRI) and the combined results from the control (nonischemic) segments (control). The shaded area represents the ischemic phase. $* p=0.002$ (IRI vs. IPC) and $p=0.045$ (IPC vs. R-IPC).

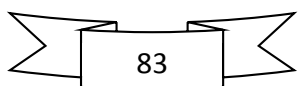




\section{Glycerol}

An approximately 7-fold increase in glycerol was observed in ischemic lobes compared with control lobes in the IRI group during the ischemic phase. Both the IPC and R-IPC groups exhibited similar patterns, but the increase in the IPC group was less pronounced than in the other two groups. At the end of ischemia $(t=80)$, glycerol was significantly lower in the IPC group (514 (70) $\mu \mathrm{M})$ in the ischemic lobe than in the IRI group (731.8 (66.8) $\mu \mathrm{M})$ and the RIPC group (759 $(84) \mu \mathrm{M})(p=0.046$ and $p=0.022$, respectively). An early significant increase in glycerol was observed after preconditioning $(\mathrm{t}=20)$ in the IPC group compared with $\mathrm{t}=0(p=0.001)$. This effect was not observed in the IRI or R-IPC groups. Glycerol, in the ischemic lobe, returned to the level of the control lobe after approximately 90 minutes of reperfusion (figure 19).

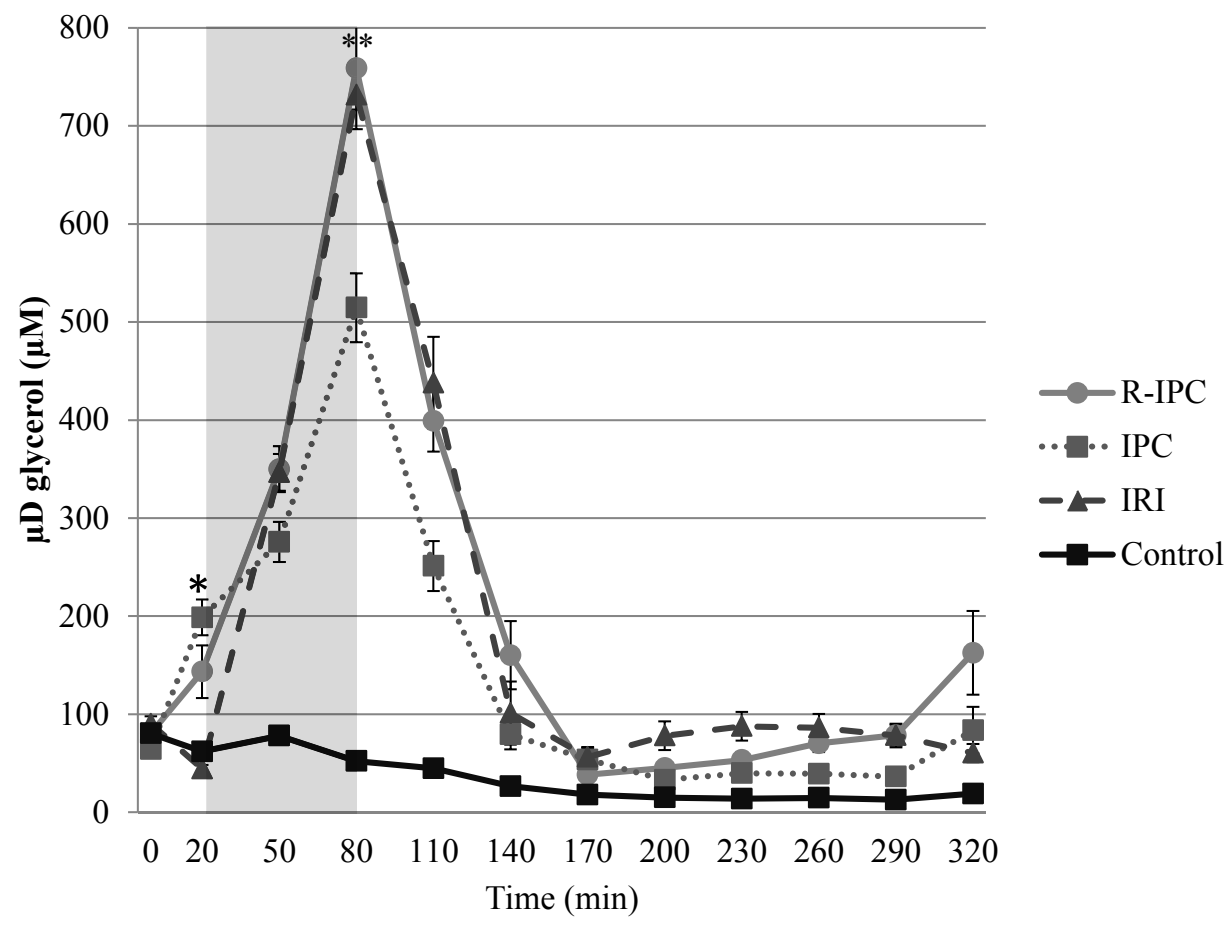

Figure 19. $\mu D$ glycerol (study I). Mean \pm SEM $\mu D$ glycerol in the ischemic liver segment during 1-hour segmental liver ischemia and 4-hour reperfusion in rats pre-treated with IPC or R-IPC compared with non-treated rats (IRI) and the combined results from the control (non-ischemic) segments (control). The shaded area represents the ischemic phase.

$* p=0.001$ ( $t=0$ min vs. $t=20$ min in IPC).

$* * p=0.046$ (IRI vs. IPC) and 0.022 (IPC vs. R-IPC).

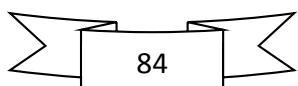




\section{Histology}

The only abnormal histological finding in the control lobes (all groups and all stratas) was slight sinusoidal congestion of the liver parenchyma (scores $0-1$ ).

In the ischemic lobes (all groups), sinusoidal congestion tended to be more obvious after 4 hours of reperfusion (scores 2-4) than in the early state (1-hour reperfusion). Histological signs of ischemia-reperfusion injury, identified as hepatocellular cytoplasmic vacuolation, sinusoidal congestion and hepatocyte necrosis, were more obviously observed in the IRI and the R-IPC groups than in the IPC group after 4-hour reperfusion. According to the grading system, the scores were higher in these 2 groups than in the IPC group. Figure 20 presents representative slides from the livers of the different groups after 4-hours reperfusion.

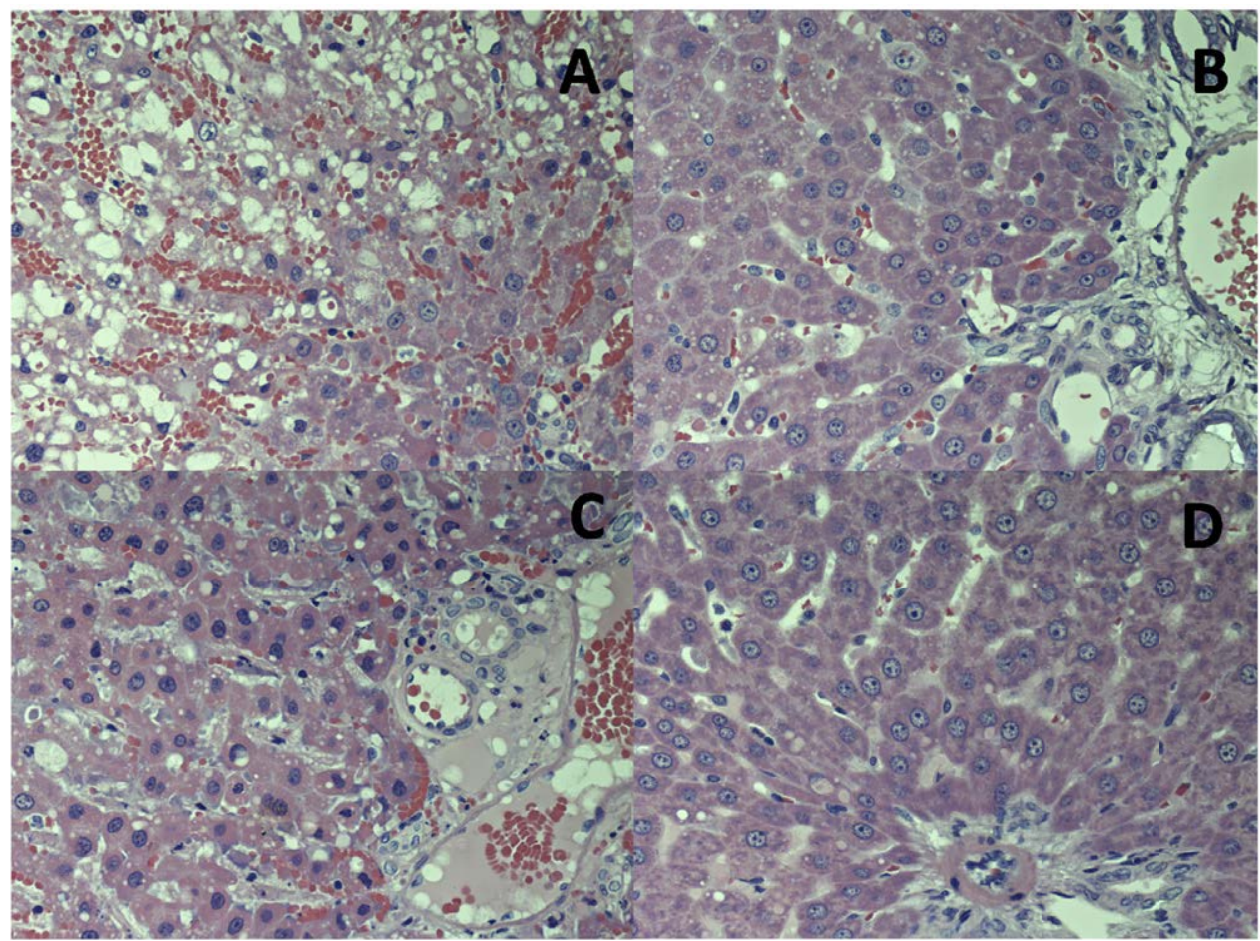

Figure 20. Histology (study I). H\&E-stained (x 40 magnification) specimens from the ischemic lobes of rat livers after 1-hour segmental ischemic followed by 4-hour reperfusion. IR only (A), IR after IPC (B), IR after R-IPC (C) and sham operated (D).

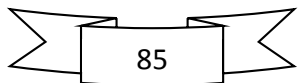




\section{Study II}

\section{Demographics}

The IPC group $(\mathrm{n}=16)$ and the control group were similar with respect to demographics (age, sex and chemotherapy before operation), operative data (bleeding, transfusion, durations of ischemia, resections volume and glycogen levels) and length of stay.

When stratified according to resection size, subjects undergoing major resection $(>3$ segments) had larger liver volumes resected than those having three or less segments resected (700 (87) vs. $184(26) \mathrm{ml}, p<0.001)$. The transectional area was also larger in major resections (122 (7) vs. $\left.93(9) \mathrm{cm}^{2}, p<0.05\right)$. The operation time was longer for the major resections group ((328 (24) vs. 251 (13) $\mathrm{min}, p<0.05)$. The length of stay was longer for major resections compared with minor resections (10 vs. 9 days, $p<0.05)$. Table 3 presents the demographic and perioperative data.

\begin{tabular}{|c|c|c|c|c|}
\hline & \multicolumn{2}{|c|}{2 - 3 segments } & \multicolumn{2}{|c|}{$>3$ segments } \\
\hline & $\mathrm{IPC}(\mathrm{n}=8)$ & Control $(\mathrm{n}=8)$ & $\operatorname{IPC}(\mathrm{n}=8)$ & Control $(\mathrm{n}=8)$ \\
\hline Sex, (\% males) & $4(50)$ & $5(62)$ & $4(50)$ & $5(62)$ \\
\hline Age (years) & $67(15)$ & $62(9)$ & $60(13)$ & $66(10)$ \\
\hline Preop glycogen levels ( $\mu \mathrm{g} / \mathrm{mg}$ fdt) & $40(6.0)$ & $42(9.8)$ & $41(7.4)$ & $37(7.7)$ \\
\hline Preop chemotherapy (n (\%)) & $4(50)$ & $3(38)$ & $7(88)$ & $6(75)$ \\
\hline Units of insulin given (total \# (range) & $51(0-47)$ & $24(0-20)$ & $84(0-60)$ & $60(0-60)$ \\
\hline LOS (days) & $7-13(9)$ & $6-11(6)$ & $8-13(10)$ & $7-51(11)$ \\
\hline Blood loss (mL) & $100-1700(359)$ & $50-2400(300)$ & $100-1500(625)$ & $200-1800(750)$ \\
\hline Units of blood transfused (total \# (range)) & $6(0-4)$ & $4(0-2)$ & $10(0-5)$ & $7(0-3)$ \\
\hline Units of plasma transfused (total \# (range)) & $9(0-7)$ & $7(0-5)$ & $13(0-6)$ & $18(0-7)$ \\
\hline Resected volume (mL) & $55-340(190)$ & $60-400(165)$ & $225-1150(698)$ & $300-1200(550)$ \\
\hline Transected area $\left(\mathrm{cm}^{2}\right)$ & $91(36)$ & $95(40)$ & $132(33)$ & $113(23)$ \\
\hline Bleeding $/ \mathrm{cm}^{2}\left(\mathrm{~mL} / \mathrm{cm}^{2}\right)$ & $1.0-11(5.2)$ & $0.5-15(5.9)$ & $0.9-25(4.4)$ & $2.3-21(6.6)$ \\
\hline Duration of ischemia (min) & $44(10)$ & $44(13)$ & $35(11)$ & $44(8.0)$ \\
\hline Transection time (min) & $64(22)$ & $77(40)$ & $62(32)$ & $60(11)$ \\
\hline Duration of Surgery (min) & $242(57)$ & $261(42)$ & $292(60)$ & $359(112)$ \\
\hline Bleeding/min (transection) $(\mathrm{mL} / \mathrm{min})$ & $1.8-19(5.7)$ & $0.9-20(6.5)$ & $2.6-36(9.2)$ & $3.9-42(11.2)$ \\
\hline Bleeding/min (total) (mL/min) & $0.5-5.2(1.8)$ & $0.6-2.9(1.1)$ & $0.4-5.2(2.0)$ & $0.9-4.7(3.6)$ \\
\hline Complications (n (\%)) & $3(38)$ & $2(25)$ & $4(50)$ & $3(38)$ \\
\hline \multicolumn{5}{|l|}{ Diagnosis: } \\
\hline Metastases (n (\%)) & $7(88)$ & $4(50)$ & $8(100)$ & $7(88)$ \\
\hline Benign (n (\%)) & $1(12)$ & $3(38)$ & 0 & 0 \\
\hline Carcinoma (HCC?) (n (\%)) & 0 & 0 & 0 & $1(12)$ \\
\hline Gall bladder cancer (n (\%)) & 0 & $1(12)$ & 0 & 9 \\
\hline Steatosis $(\mathrm{n}(\%))$ & $1(12)$ & 0 & 0 & $3(38)$ \\
\hline Portal fibrosis (n (\%)) & $1(12)$ & 0 & 0 & 0 \\
\hline
\end{tabular}

Table 3. Demographic and perioperative data (study II). Patients underwent operations for resections of either 2-3 liver segments or $>3$ liver segments with or without pre-treatment with IPC.

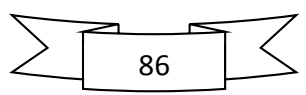




\section{Diagnosis and complications}

The most common diagnosis was liver metastases $(n=26)$ followed by benign lesions $(n=4)$, gallbladder cancer $(n=1)$ and hepatocellular cancer $(n=1)$.

Complications occurred in $12(37.5 \%)$ patients, and no differences were noted between the groups (table 3).

\section{Blood analyses}

When serum and blood analyses were compared, no difference was observed between the IP group and controls. However, patients treated with IPC before major resections exhibited reduced levels of lactate $(2.4(0.1)$ vs. $4.4(0.5) \mathrm{mM}, p<0.05)$ and INR (1.2 (0.04) vs. 1.5 $(0.04) p<0.05)$ immediately postoperatively compared with controls.

\section{Microdialysis}

\section{Minor resections}

The only difference noted between the treatment and control groups undergoing minor resections was that glucose was reduced in the treatment group on POD 2 compared with the control group.

\section{Major resections}

Immediately after surgery and up to POD 1 , the levels of $\mu \mathrm{D}$-glucose, $\mu \mathrm{D}$-pyruvate and $\mu \mathrm{D}$ lactate were lower in the IPC group compared with the controls. 
Glucose was reduced in the IPC-treated group compared with the control group (4.9 (0.1) vs. $6.6(0.1) \mathrm{mM}, p<0.001)$ on the operation day (figure 21). In addition, on POD 2, glucose was lower $(6.1(0.1)$ vs. $8.3(0.2) \mathrm{mM}, p<0.05)$ in patients treated with IPC before major resection compared with controls.

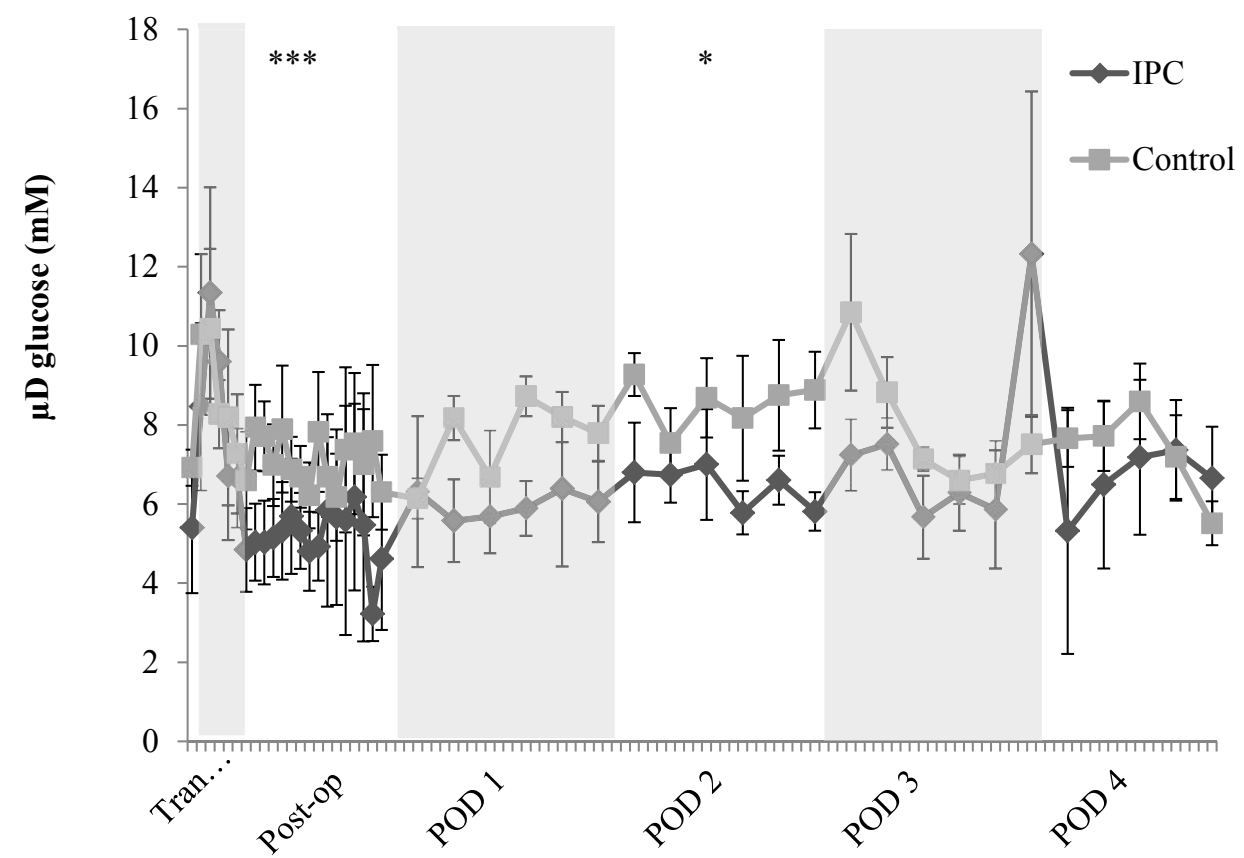

Figure 21. $\mu D$ glucose (study II). Mean \pm SEM $\mu D$ glucose levels in patients undergoing major liver resection with IPC and without IPC (control) before intermittent PM.

Tran ... = Transection phase.

$* * * p<0.001$.

$* p<0.05$.

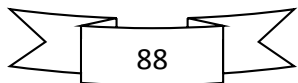


Similarly, the pyruvate level was lower in the IPC-treated group than in controls (147 (3.2) vs. 198 (3.5) $\mu \mathrm{M}, p<0.001$ ) on the operation day (figure 22). From POD 1 onwards, no significant differences in the pyruvate levels were noted between the groups.

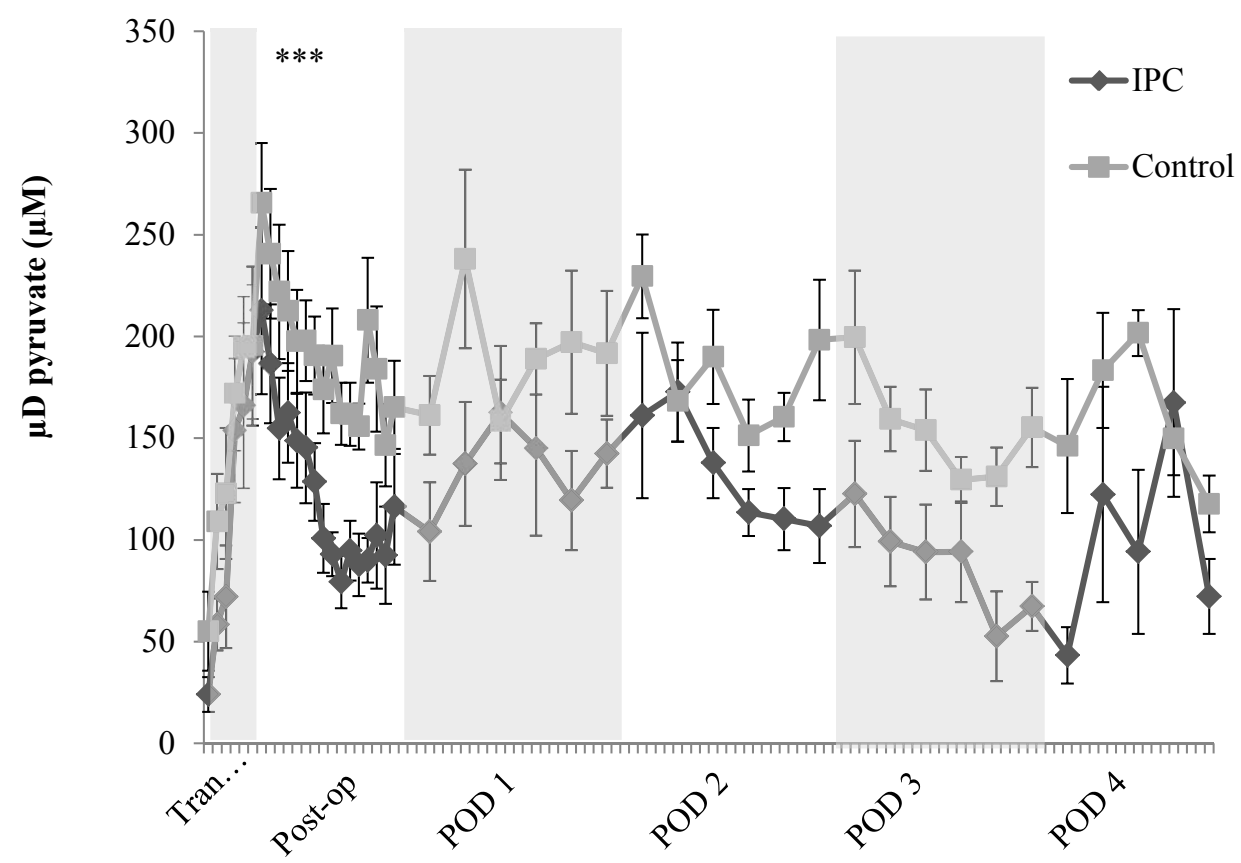

Figure 22. $\mu$ D pyruvate (study II). Mean \pm SEM $\mu$ D pyruvate levels in patients undergoing major liver resection with IPC and without (control) IPC before intermittent PM.

Tran ... $=$ Transection phase

$* * *=p<0.001$.

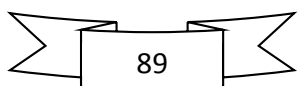


Lower lactate levels were observed during the transection phase in IP-treated patients undergoing major resections than in controls $(4.25(0.12)$ vs. $5.8(0.13) \mathrm{mM}, p<0.001)$. This difference continued through the entire day of operation $(2.6(0.06) v s .4 .0(0.08) \mathrm{mM}, p<$ $0.001)$ and was also observed on POD 2 (1.8 (0.05) vs. 2.3 (0.06) $\mathrm{mM} p<0.05$ ) (figure 23).

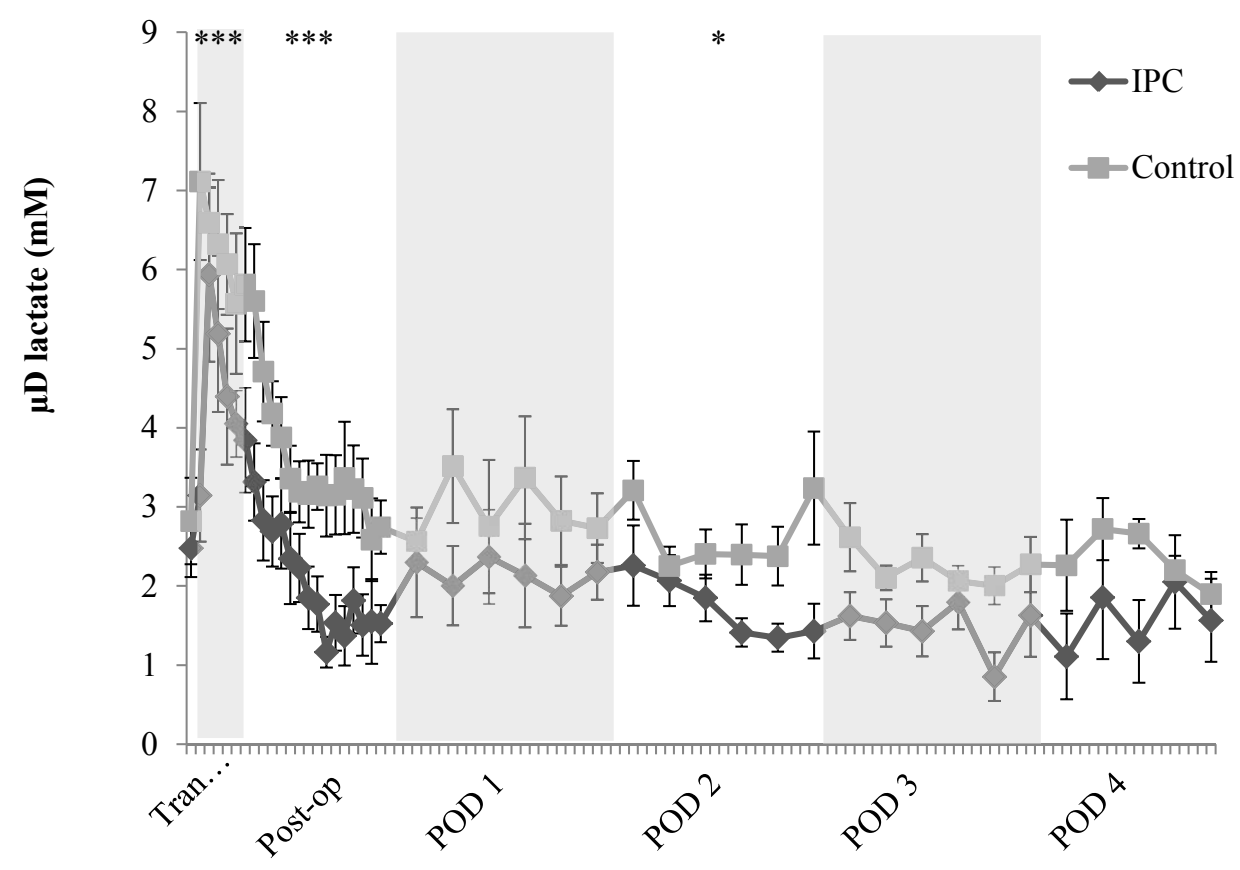

Figure 23. $\mu D$ lactate (study II). Mean (SD) $\mu D$ lactate levels in patients undergoing major liver resection with and without IPC before intermittent PM.

Tran ... = Transection phase.

$* * *=p<0.001$.

$*=p<0.05$.

Glycerol levels did not differ in $\mu \mathrm{D}$ between the groups, and no difference was found between major and minor resections.

$\mathrm{NO}_{\mathrm{x}}$ levels decreased continuously postoperatively in the IPC group from 31 (3) to 22 (3) $\mu \mathrm{M}$ $(p<0.01)$ up to POD 3, when the levels started to return to the levels seen before surgery. In the control group, a similar pattern was observed, and no difference was noted between the groups. The $\mathrm{NO}_{\mathrm{x}}$ levels in the $\mu \mathrm{D}$ were not affected by the volume of the resection.

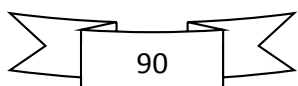




\section{Glycogen}

No difference in glycogen levels was noted between the groups or between major and minor resections. Male patients exhibited increased glycogen levels compared with females (45.0 (0.5) vs. $36.7(0.5) \mu \mathrm{g} / \mathrm{mg}$ freeze-dried tissue, $p<0.05)$.

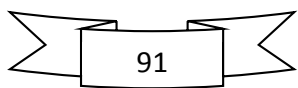




\section{Study III}

NOx in serum

In the R-IPC group, an initial rise $(20.0$ to $25.2 \mu \mathrm{M})$ during ischemia and 1-hour reperfusion followed by a decline ( 25.2 to $23.7 \mu \mathrm{M}$ ) during later parts of reperfusion was observed. In the IPC group, the rise $(20.2$ to $27.7 \mu \mathrm{M})$ was more pronounced early than in the R-IPC group (ns), but the decline (27.7 to $23.4 \mu \mathrm{M}$ ) started directly in the reperfusion phase (figure 24). In both preconditioning groups, the rise and decline in S-NOx was statistically significant (both groups $p<0.001$ ), but no significant difference was noted between the two groups.

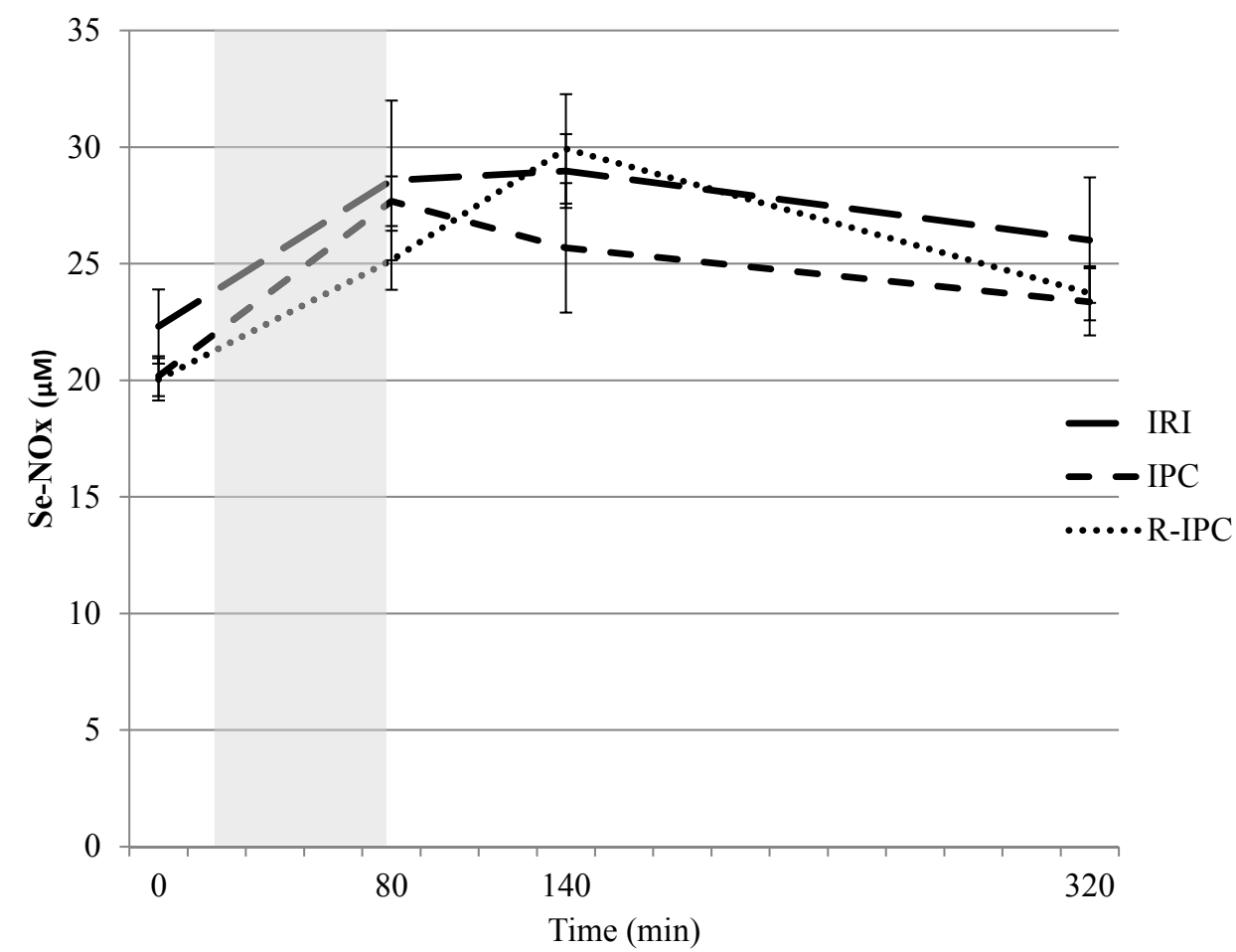

Figure 24. Serum NOx (study III). Mean \pm SEM se-NOx in rats treated with IPC, R-IPC or no preconditioning before 1-hour segmental liver ischemia followed by 4-hour reperfusion. The shaded area represents ischemic phase.

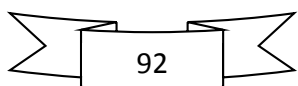




\section{NOx in microdialysate fluid}

In both the IPC (11.8 to $6.4 \mu \mathrm{M})$ and R-IPC (12.3 to $4.7 \mu \mathrm{M})$ groups, significant decreases in $\mathrm{NOx}$ in the $\mu \mathrm{D}$ fluid were observed at the end of ischemia $(p=0.007$ and $p=0.002$, respectively) compared with the levels in early ischemia. In both groups, the levels quickly increased again during reperfusion (figure 25). In the control lobes, only small variations were observed, and no difference was noted between the groups (data not shown).

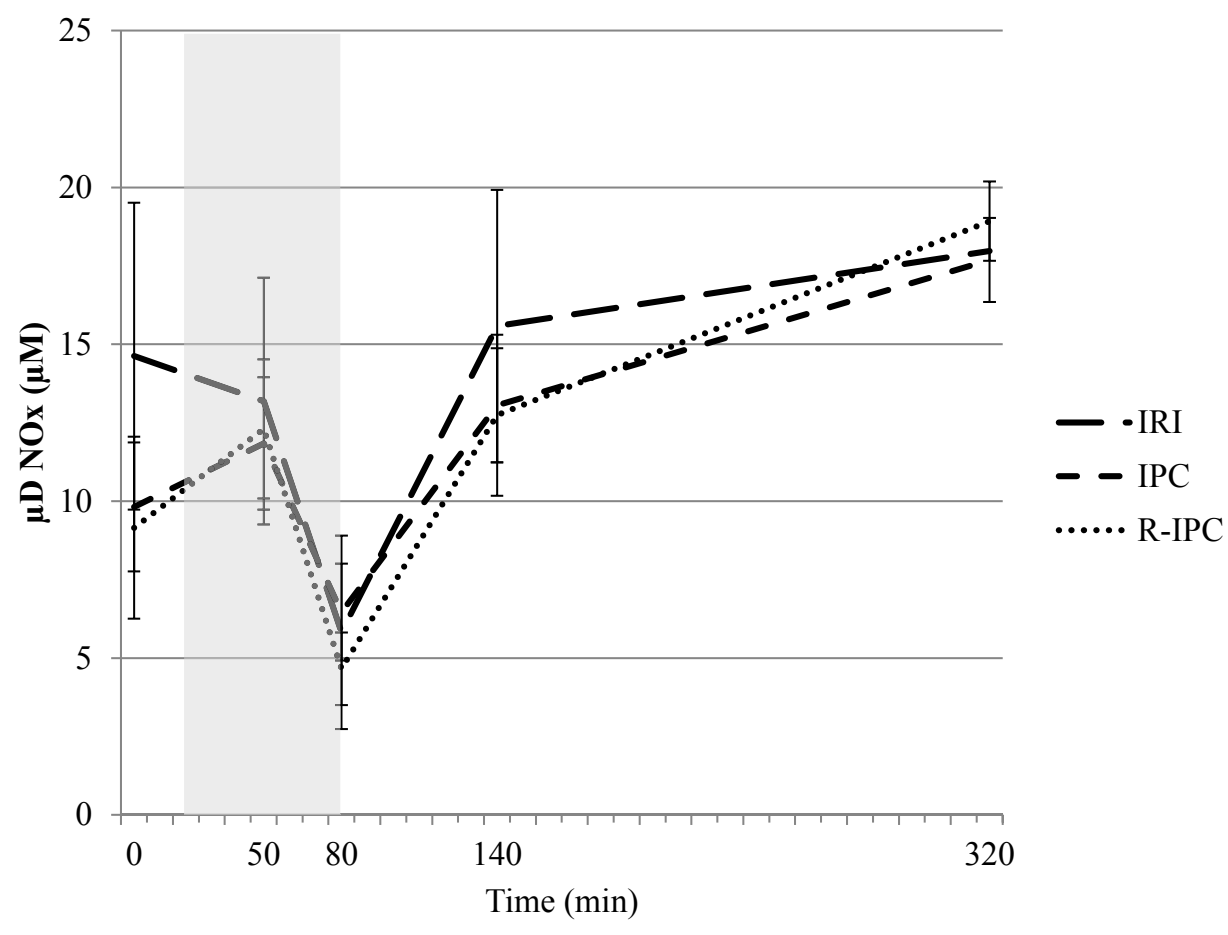

Figure 25. $\mu D$ NOx (study III). Mean \pm SEM NOx in the microdialysate in rats treated with $I P C, R-I P C$ or no preconditioning before 1-hour segmental liver ischemia followed by 4-hour reperfusion. The shaded area represents ischemic phase.

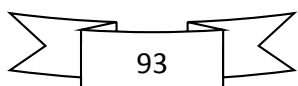


iNOS mRNA in liver tissue

iNOS transcription at the end of reperfusion was significantly increased in both the IPC $(\triangle \mathrm{Ct}$ 5.86) and R-IPC ( $\Delta \mathrm{Ct} 3.44)$ groups compared with levels at the end of ischemia ( $\Delta \mathrm{Ct} 10.78$ and 11.49 , respectively) ( $p<0.001$ in both groups) (figure 26). When the groups were compared, level of iNOS mRA was significantly higher in the R-IPC group than in the IPC group at the end of reperfusion $(p=0.03)$.

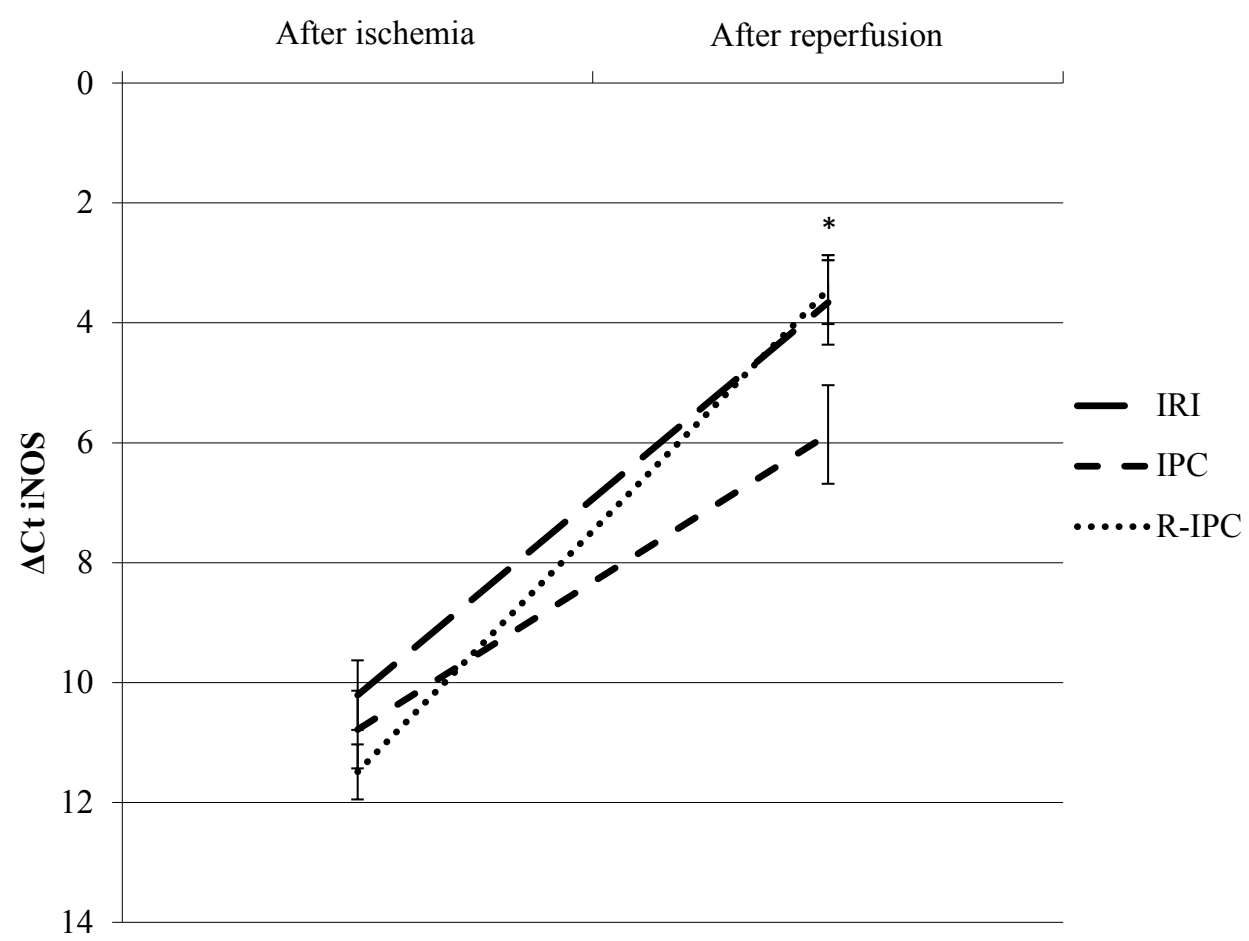

Figure 26. iNOS transcription (study III). Mean \pm SEM iNOS mRNA in liver tissue from rats treated with IPC, R-IPC or no preconditioning before 1-hour segmental liver ischemia followed by 4-hour reperfusion.

$*=p<0.05$.

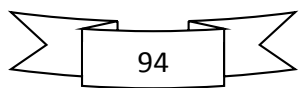




\section{IL-1 receptor $m R N A$ in liver tissue}

IL-1 receptor expression was measured with semi-quantitative real-time PCR. In the IPC group, a trend towards reduced IL-1 transcription ( $\Delta \mathrm{Ct} 1.88$ to 4.81 ) was noted during reperfusion, although this trend did not reach statistical significance $(p=0.0636)$. In the $\mathrm{R}$ IPC group, this decrease was not observed given that the transcriptional activity increased slightly ( $\Delta \mathrm{Ct} 3.26$ to 2.99 ) (ns) (figure 27 ).

When IPC and R-IPC were compared, the IPC group exhibited significantly increased IL-1 receptor mRNA levels at the end of ischemia $(p=0.0034)$. At the end of reperfusion, the R-IPC group exhibited a trend towards higher transcription of IL-1 receptor mRNA compared with the IPC group; however, this difference was not statistically significant $(p=0.273)$.

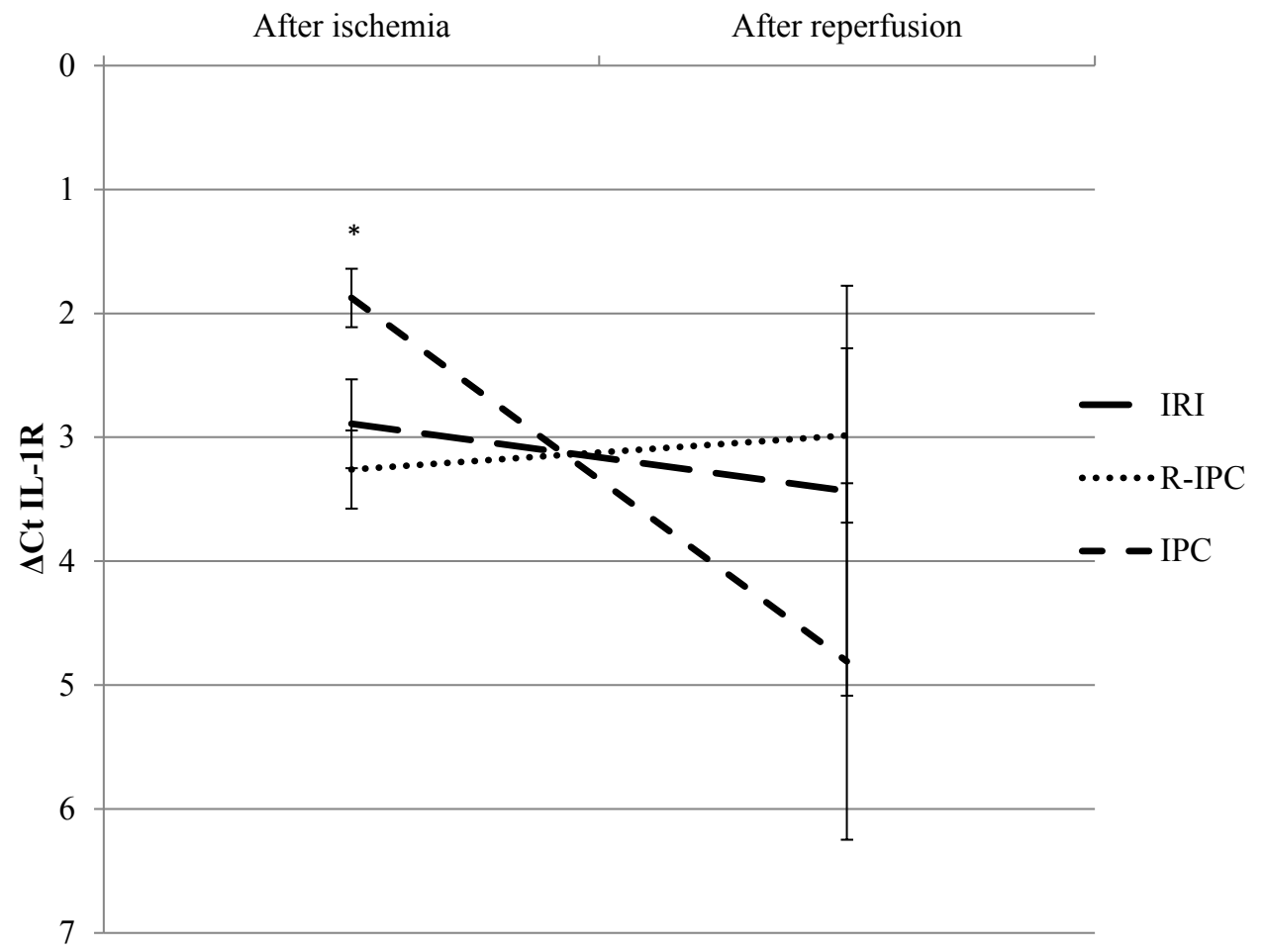

Figure 27. IL-1R transcription (study III). Mean \pm SEM IL-1R $m R N A$ in liver tissue from rats treated with IPC, R-IPC or no preconditioning before 1-hour segmental liver ischemia followed by 4-hour reperfusion. $*=p<0.05$ (IPC vs. R-IPC).

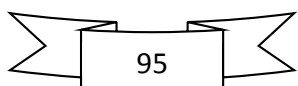




\section{Study IV}

Blood analyses

\section{Liver transaminases}

Higher serum AST levels (figure 28) were observed in the IRI group (40 (6.8) $\mu \mathrm{kat} / \mathrm{l})$ after 4 hours of reperfusion than in the nitrite-treated group (22 (2.6) $\mu \mathrm{kat} / \mathrm{l})(p=0.022)$.

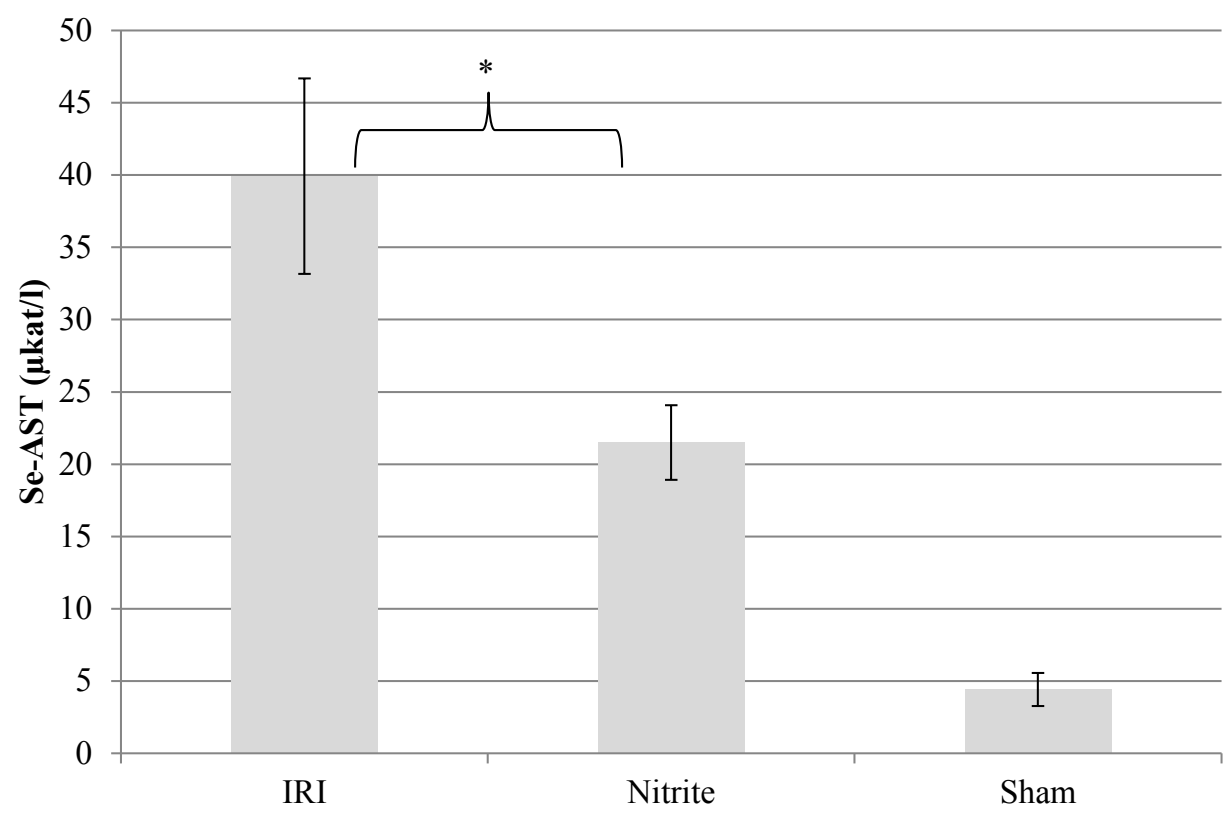

Figure 28. Serum AST (study IV). Mean $\pm S E M$ se-AST in rats subjected to 45-minute segmental (left lateral lobe) liver ischemia and 4-hour reperfusion with (Nitrite) or without (IRI) pre-treatment with $480 \mathrm{nmol}$ nitrite intravenously. Animals treated with nitrite before ischemia and reperfusion exhibited significantly lower AST levels than untreated animals.

$* p=0.022$.

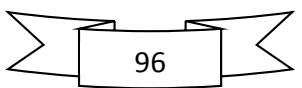


ALT levels (figure 29) at 4 hours of reperfusion were significantly increased in the IRI group (34 (6) $\mu \mathrm{kat} / \mathrm{l})$ compared with the nitrite group (14 (1.5) $\mu \mathrm{kat} / \mathrm{l})(p=0.0045)$. AST and ALT levels were approximately normal after reperfusion in the sham group.

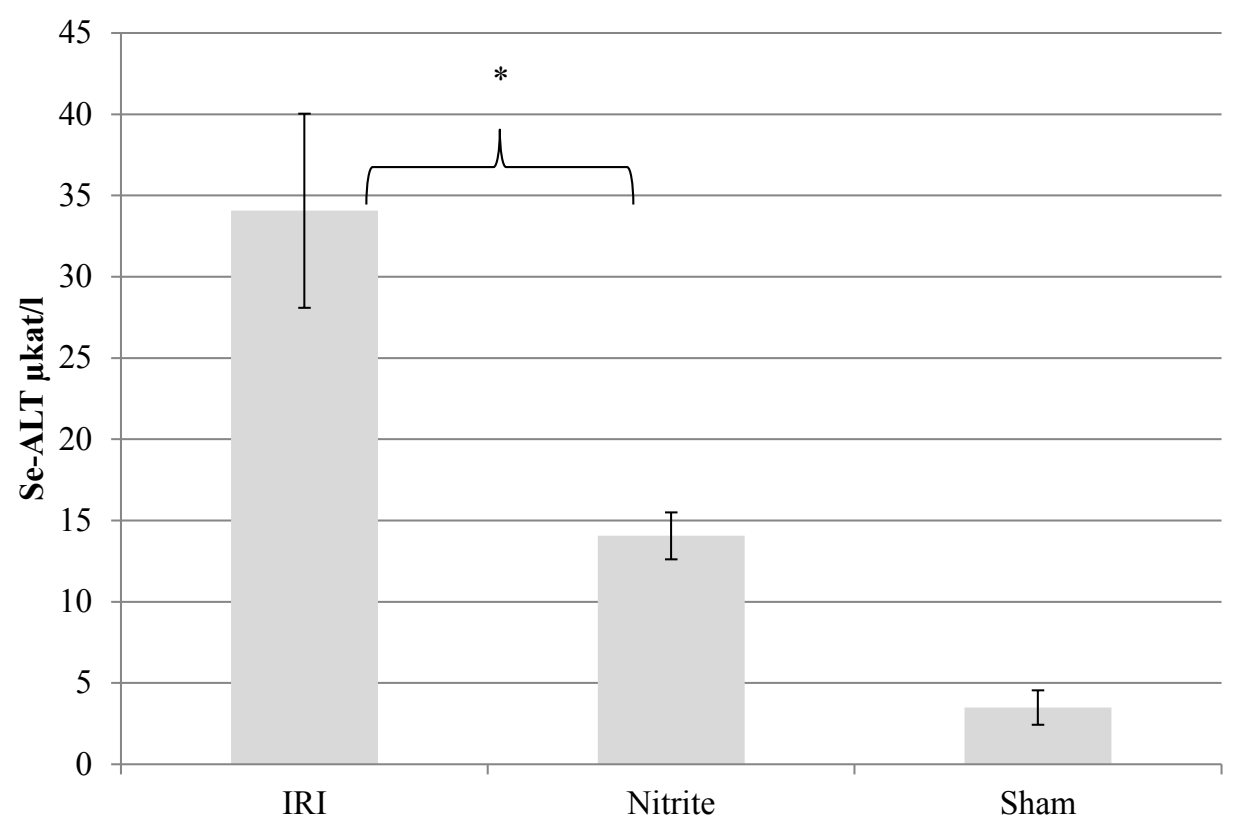

Figure 29. Serum ALT (study IV). Mean \pm SEM se-ALT in rats subjected to 45-minute segmental (left lateral lobe) liver ischemia and 4-hour reperfusion with (Nitrite) or without (IRI) pre-treatment with $480 \mathrm{nmol}$ nitrite intravenously. Animals treated with nitrite before ischemia and reperfusion exhibited significantly lower AST levels than untreated animals.

$* p=0.0045$.

$\underline{\mathrm{NOx}}$

The sum of nitrite and nitrate (NOx) measured in the serum at the end of reperfusion did not significantly differ between the groups.

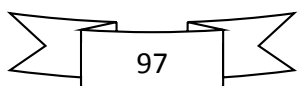


Parenchymal metabolism measured by microdialysis

NOx

NOx in the liver tissue measured via microdialysis was significantly $(p=0.031)$ increased in the nitrite group $(10.1 \pm 2.9 \mu \mathrm{M})$ compared with the IRI group $(3.2 \pm 0.9 \mu \mathrm{M})$ after the administration of nitrite (figure 30). During ischemia, the levels of NOx decreased in both groups and thereafter increased during reperfusion. After 4-hour reperfusion, a trend towards increased levels of NOx in the IRI group compared with the nitrite group was noted ( $p=$ 0.067).

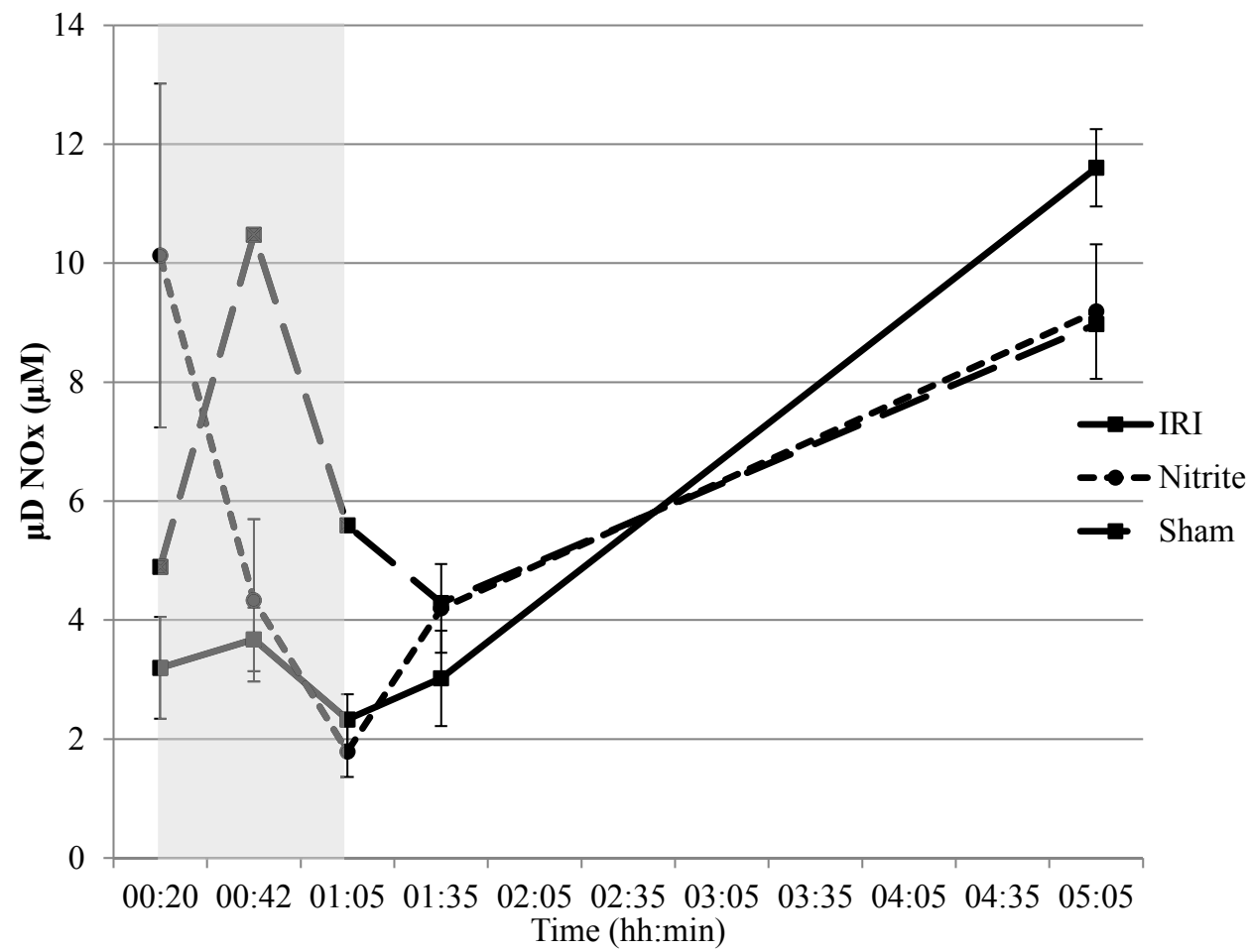

Figure 30. $\mu D$ NOx (study IV). Parenchymal (microdialysis) NOx (nitrite and nitrate) (mean $\pm S E M)$ in the ischemic liver lobe in rats subjected to 45-minute segmental (left lateral lobe) liver ischemia and 4-hour reperfusion with (Nitrite) or without (IRI) pre-treatment with 480 nmol nitrite intravenously. After the administration of nitrite, parenchymal NOx was increased compared with animals not receiving nitrite $(p=0.031)$. During ischemia, the levels decreased in both groups and then increased again during reperfusion. After 4-hour reperfusion, a tendency towards increased levels in animals not treated with nitrite was noted ( $p=0.067)$. The shaded area represents the ischemic phase.

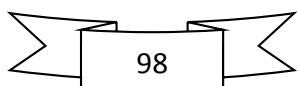




\section{Glucose}

In the both groups, glucose increased approximately 2 fold (6 to $12 \mathrm{mM}$ ) during ischemia in the ischemic lobe compared with the control lobe as well as the pre-ischemic value in the ischemic lobe. However, no significant difference was observed between the groups (figure $31)$.

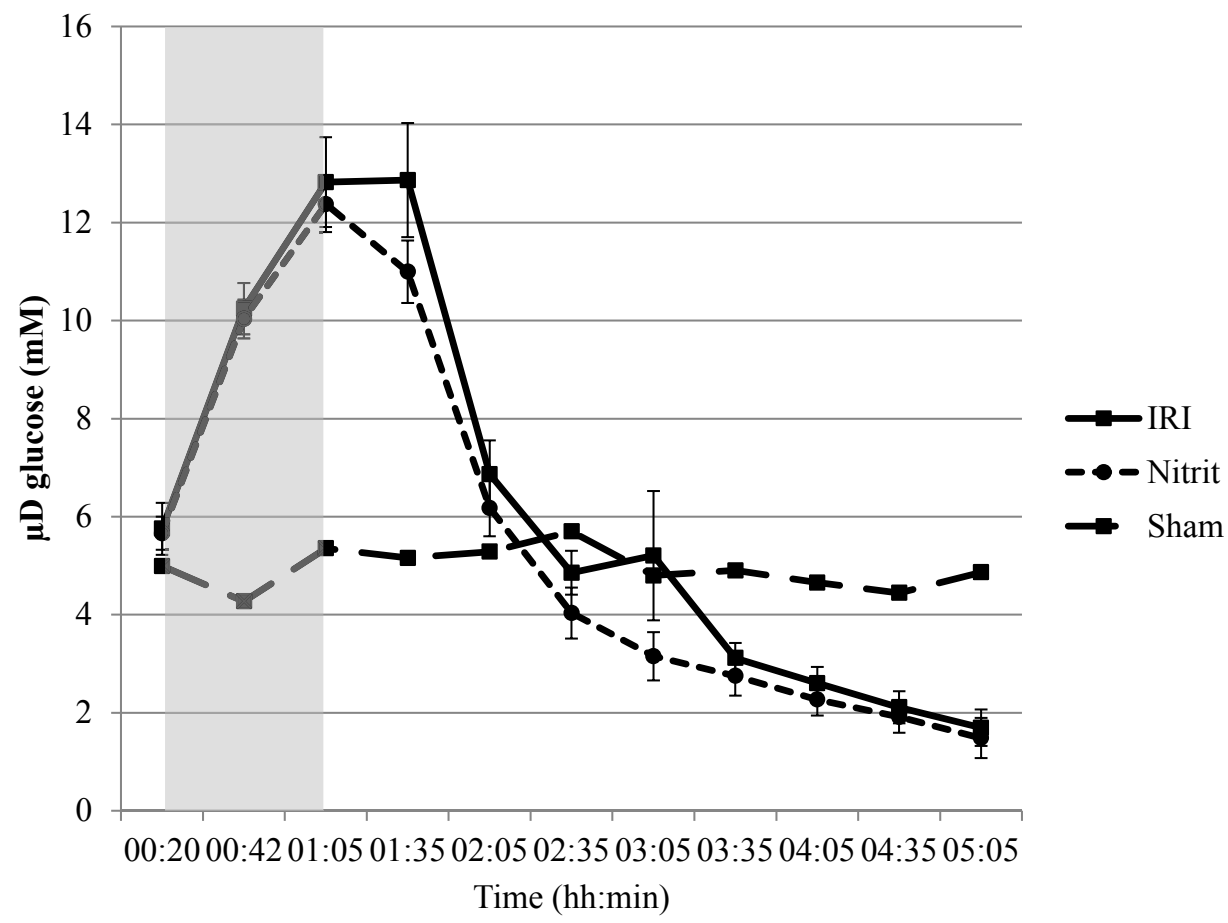

Figure 31. $\mu D$ glucose in the ischemic lobe (study IV). Parenchymal (microdialysis) glucose (mean $\pm S E M)$ in the ischemic liver lobe in rats subjected to 45-minute segmental (left lateral lobe) liver ischemia and 4-hour reperfusion with (Nitrite) or without (IRI) pre-treatment with $480 \mathrm{nmol}$ nitrite intravenously. During ischemia, parenchymal glucose increased in both groups, and no difference was noted between the groups. In the reperfusion phase, a steady decline in glucose levels was noted. The shaded area represents the ischemic phase.

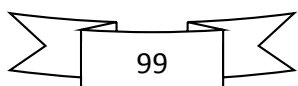


In the control lobe in the nitrite group, a significant $(p<0.001)$ increase $(6.1$ to $7.7 \mathrm{mM})$ in glucose was observed after nitrite administration. At $\mathrm{t}=00: 43$, during ischemia and early (30 minutes) reperfusion, the difference between the groups was significant ( $p=0.049$ and $p=$ 0.02 , respectively) (figure 32 ).

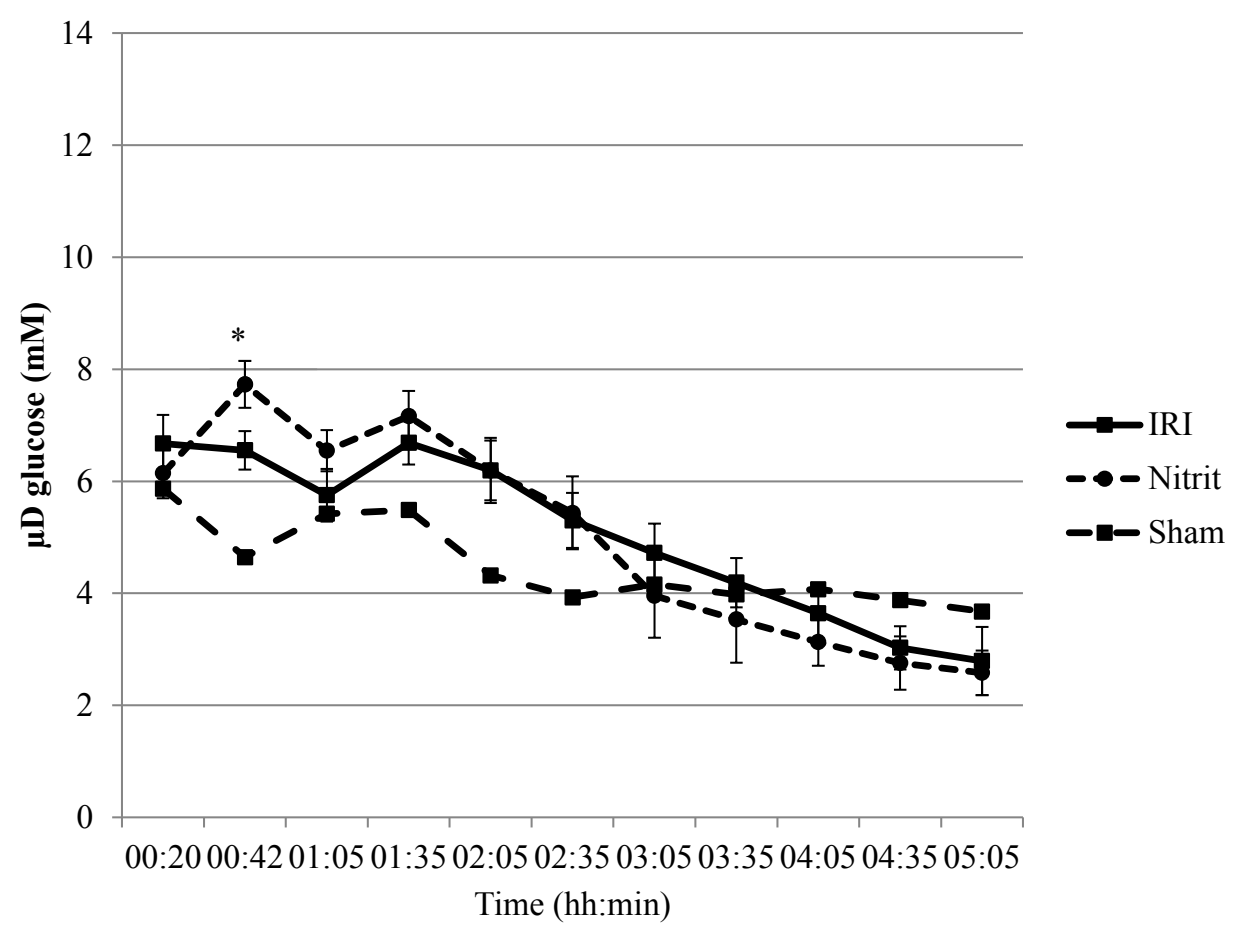

Figure 32. $\mu D$ glucose in the control lobe (study IV). Parenchymal (microdialysis) glucose (mean $\pm S E M)$ in the control (non-ischemic, right) liver lobe in rats subjected to 45-minute segmental (left lateral lobe) liver ischemia and 4-hour reperfusion with (Nitrite) or without (IRI) pre-treatment with $480 \mathrm{nmol}$ nitrite intravenously. After the administration of nitrite, parenchymal glucose increased significantly $(p<0.001)$ and achieved levels higher than in the untreated group $(p=0.02)$.

$* p<0.001$ ( $t=00: 20$ vs. $t=00: 42$ in Nitrite) and $p=0.02$ (IRI vs. Nitrite).

\section{Pyruvate}

In the IRI group, pyruvate in the ischemic segment was reduced to approximately $40 \%$ of the level in the control segment at the end of ischemia. In the nitrite group, the corresponding value was $15 \%$. In the early phase of reperfusion, there was a transient increase in both groups. However, large interindividual variations were observed in the pyruvate levels and no significant differences were noted.

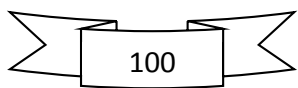




\section{$\underline{\text { Lactate }}$}

The lactate levels increased more than four fold in the ischemic lobe (4 mM) compared with the control lobe $(0.7 \mathrm{mM})$ in the IRI group during the ischemic phase. The nitrite group followed similar pattern (3.3 and $1 \mathrm{mM}$, respectively), although the change was less in magnitude.

During ischemia and during the first $30 \mathrm{~min}$ of reperfusion, lactate was significantly increased in the IRI group compared with the nitrite group ( $p=0.01)$ (figure 33).

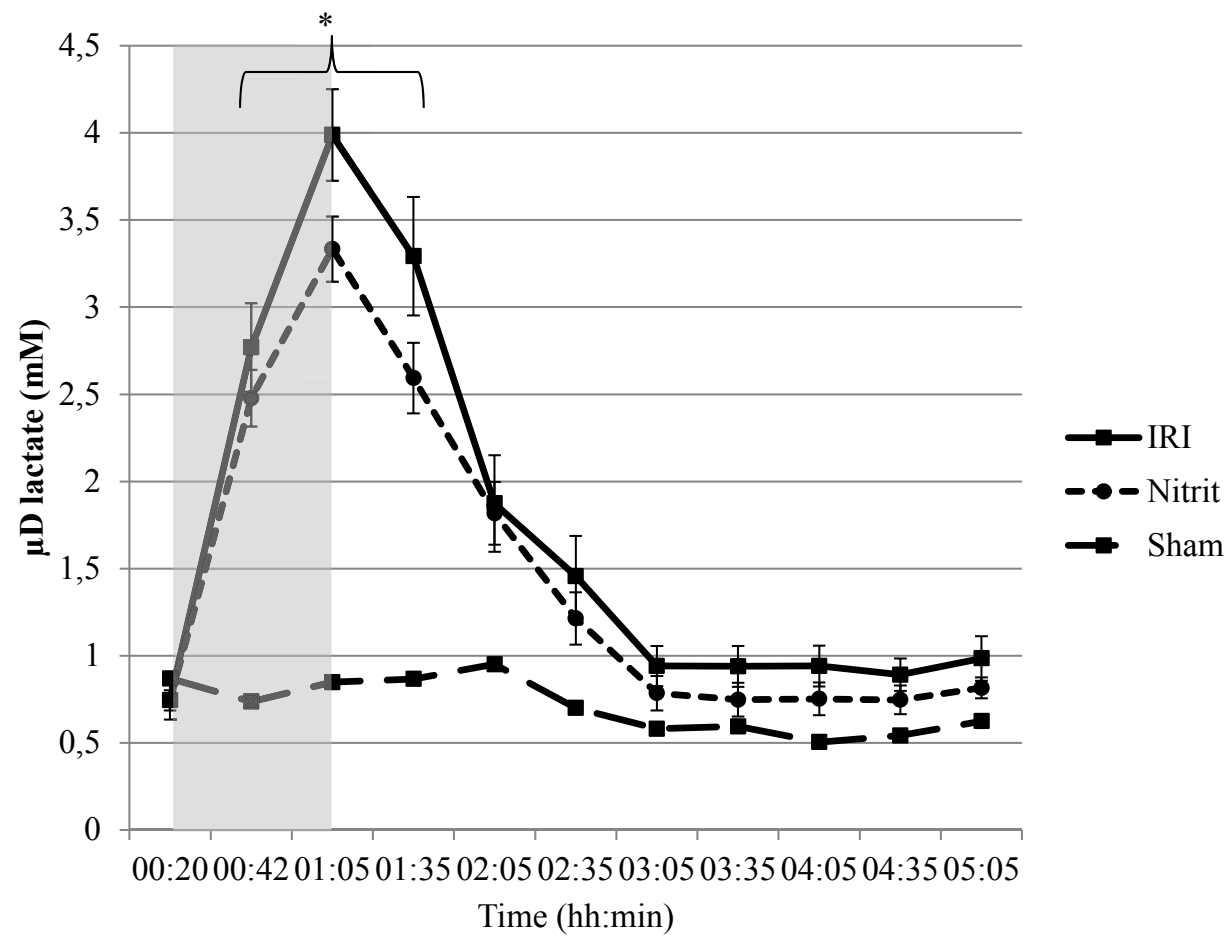

Figure 33. $\mu D$ lactate in the ischemic lobe (study IV). Parenchymal (microdialysis) lactate (mean $\pm S E M)$ in the ischemic liver lobe in rats subjected to 45-minute segmental (left lateral lobe) liver ischemia and 4-hour reperfusion with (Nitrite) or without (IRI) pre-treatment with $480 \mathrm{nmol}$ nitrite intravenously. During ischemia, parenchymal lactate increased in both groups, and during the ischemic phase and the first 30 minutes of reperfusion, the levels were increased in the untreated group than in the treated animals. The shaded area represents the ischemic phase.

$* p=0.01$ (IRI vs. Nitrite).

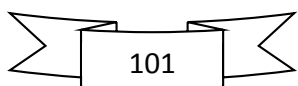


Lactate increased from 0.9 to $1.2 \mathrm{mM}(p=0.012)$ in the control lobe in the nitrite group after the administration of nitrite; this effect that was not observed in the IRI group. Furthermore, the levels of lactate were significantly $(p=0.01)$ increased during the first 95 minutes of the experiment in the control lobe in the nitrite group compared with the IRI group (figure 34).

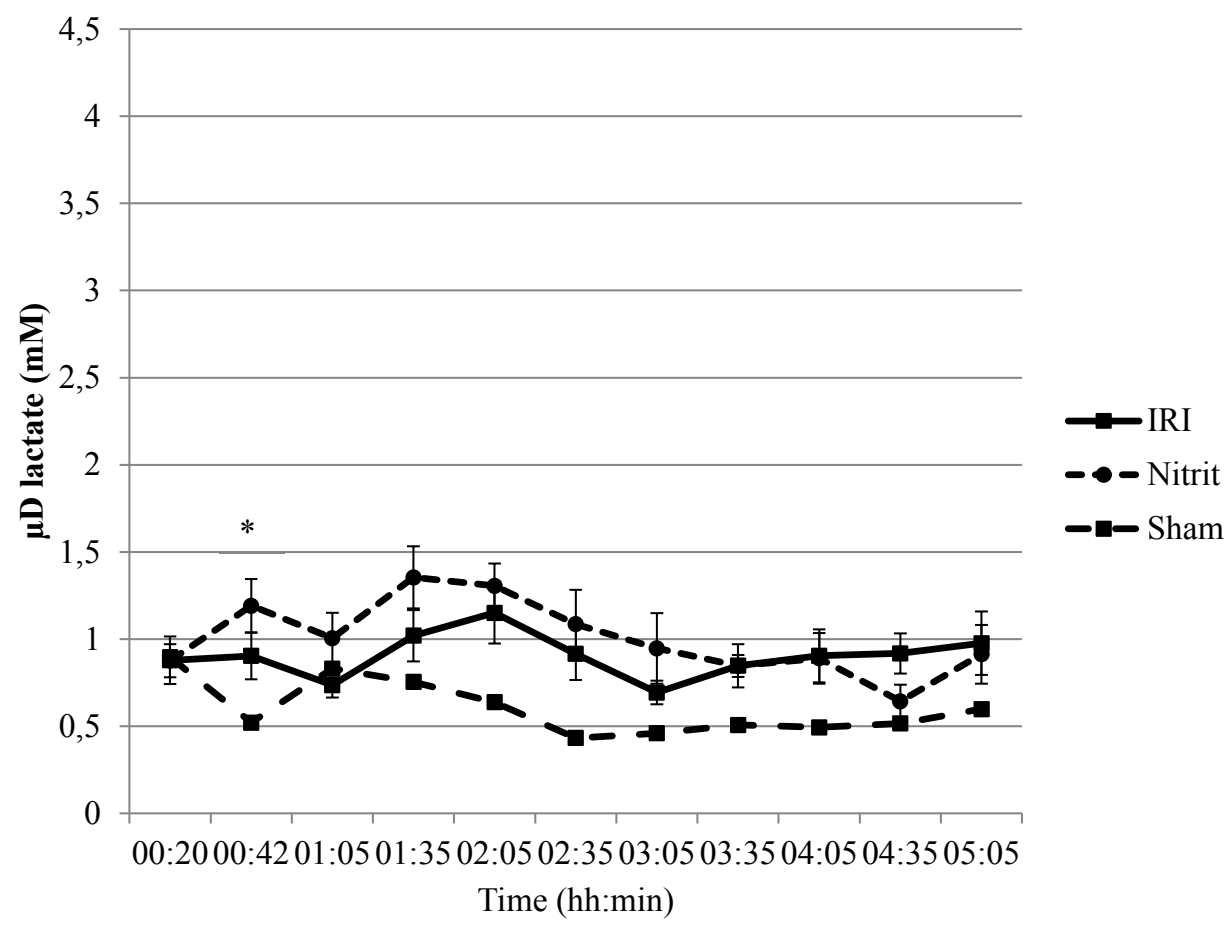

Figure 34. $\mu D$ lactate in the control lobe (study IV). Parenchymal (microdialysis) lactate (mean $\pm S E M)$ in the control (non-ischemic, right) liver lobe in rats subjected to 45-minute segmental (left lateral lobe) liver ischemia and 4-hour reperfusion with or without pretreatment with $480 \mathrm{nmol}$ nitrite intravenously. After the administration of nitrite, parenchymal lactate increased significantly $(p=0.012)$ and achieved levels higher than in the untreated group $(p=0.01)$.

$* p=0.012(t=00: 20$ vs. $t=00: 42$ Nitrite) and $p=0.01$ (IRI vs. Nitrite).

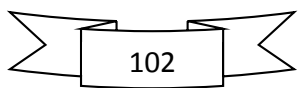




\section{Glycerol}

An approximately 38 -fold increase in glycerol in the ischemic lobe (18 to $686 \mu \mathrm{M})$ was noted in the IRI group compared with the control lobe. The nitrite group exhibited the same pattern, but the increase ( 25 to $602 \mu \mathrm{M}$ ) was less pronounced than that observed in the IRI group (24fold).

During the ischemic phase and during the early 30 minutes of the reperfusion phase, glycerol levels were significantly higher in the IRI group than in the nitrite group $(p=0.049)$. In the ischemic lobe, the glycerol level returned to the level of the control lobe after approximately 60 minutes of reperfusion (figure 35).

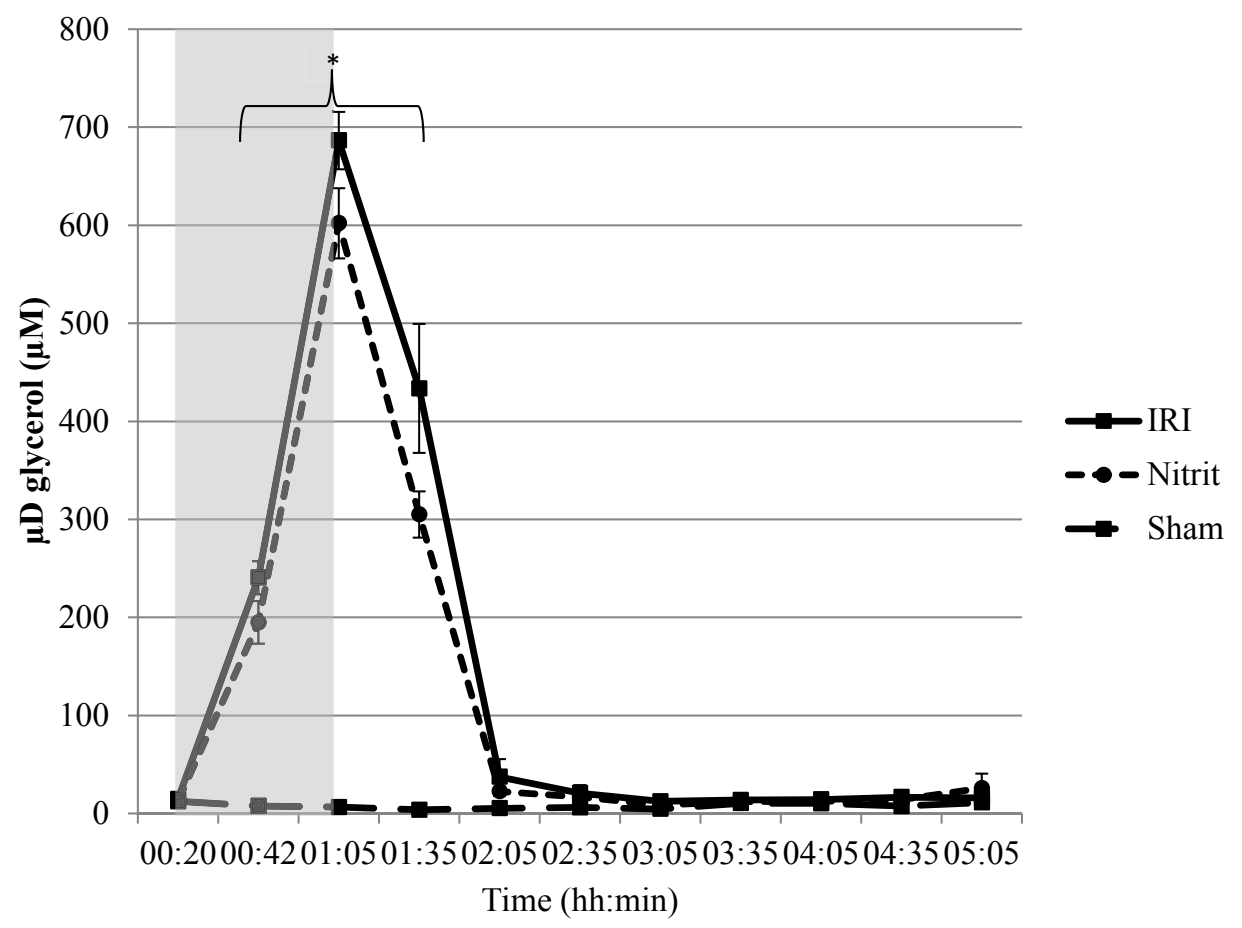

Figure 35. $\mu D$ glycerol (study IV). Parenchymal (microdialysis) glycerol (mean $\pm S E M$ ) in the ischemic liver lobe in rats subjected to 45-minute segmental (left lateral lobe) liver ischemia and 4-hour reperfusion with or without pre-treatment with 480 nmol nitrite intravenously. During ischemia, parenchymal glycerol increased in both groups. During the ischemic phase and the first 30 minutes of reperfusion, the levels were increased in the nontreated group $(p=0.049)$. The shaded area represents the ischemic phase.

$* p=0.049$ (IRI vs. Nitrite).

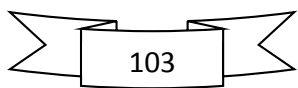


In the sham group, no significant differences were noted between the two lobes in the microdialysate (data not shown).

\section{iNOS mRNA}

Fifteen minutes into the ischemic phase, no difference in iNOS mRNA was noted between the groups (IRI $\Delta \mathrm{Ct}=9.3 \pm 0.72, \Delta \mathrm{Ct}$ nitrite $=8.6 \pm 0.24$ ). In both groups, iNOS transcription increased at the end of the experiment to $\Delta \mathrm{Ct}=3.8 \pm 0.52$ in the IRI group and $3.4 \pm 0.26$ in the nitrite group ( $p<0.01$ in both groups). However, no significant difference was noted between the groups.

\section{Histology}

All sham animals exhibited only zero scores except for 1 animal that scored 1 (10 - 40\% of hepatic tissue) with respect to sinusoidal congestion. In the IRI group, the mean score for sinusoidal congestion was 1.29 compared with 0.86 in the nitrite group. The mean score for cytoplasmic vacuolization was also higher in the IRI group (0.71) than in the nitrite group (0.43). Of the 14 animals in the IRI group, 4 (29\%) exhibited signs of necrosis on histology, whereas no animals in the nitrite group exhibited necrosis (figure 36).

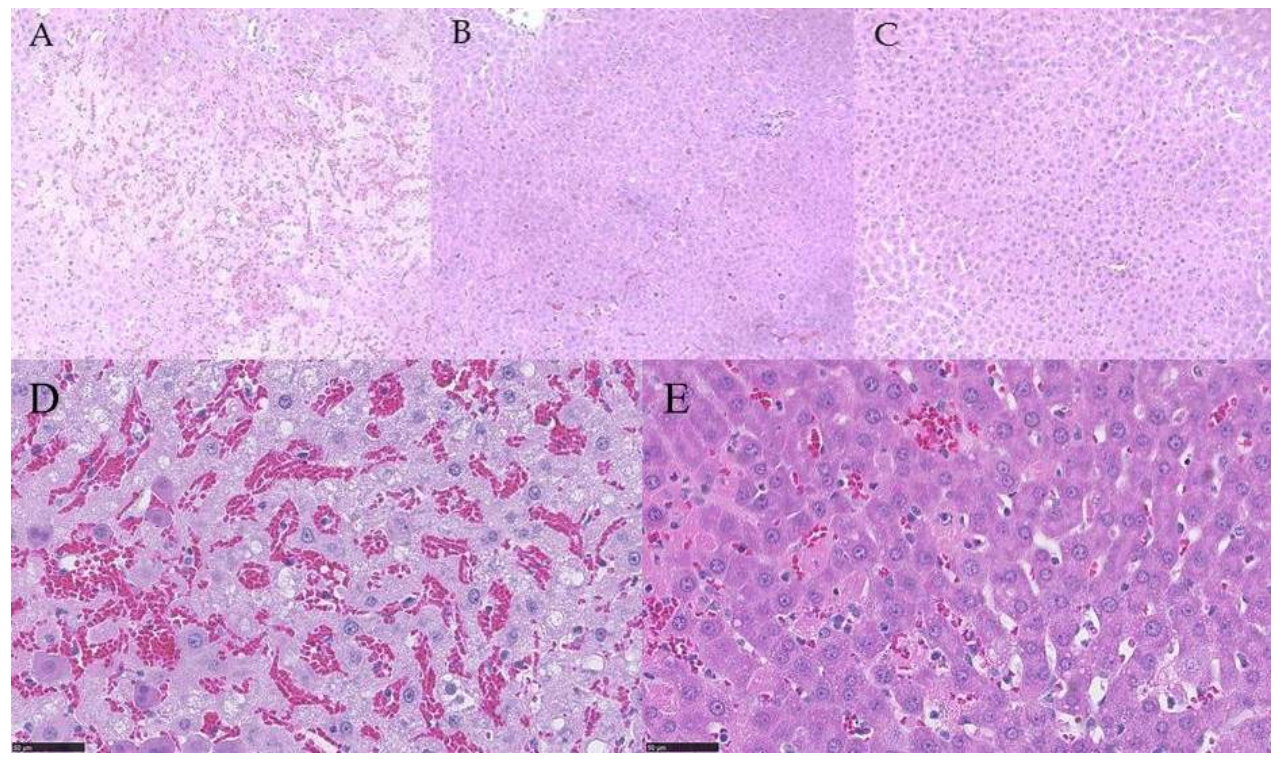

Figure 36. Histology (study IV). H\&E-stained liver tissue from the ischemic liver lobes in rats subjected to 45-minute segmental (left lateral lobe) ischemia and 4-hour reperfusion. (A and D) Without pre-treatment, ( $B$ and E) with 480 nmol nitrite administered intravenously before ischemia and (C) sham operated. A-C $20 \times$ magnification, D and E $40 \times$ magnification.

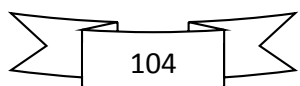




\section{Discussion}

This thesis focuses on methods to prevent or reduce IRI in the liver. As a secondary focus, this thesis explores the use of microdialysis as a tool for continuously monitoring IRI.

In liver surgery, some degree of I/R is often inevitable, and it has become clear that IRI may have detrimental effects on clinical outcomes. As liver surgery continues to increase, both in terms of the number of patients undergoing operation as well as in the complexity of the procedures, this phenomenon has gained much interest in recent decades. This increasing interest has led to a large amount of research, both in the pursuit of increased understanding of the phenomenon and to identify methods to diminish its effect. Although this search for understanding has lasted over 20 years, much is still unknown about the mechanisms involved, and no reliable method to abolish the injury has been described. Hepatic IRI is known to be a multifactorial cascade involving many cell types and substances. As the process is complex and dynamic, results are often divergent and difficult to extrapolate to other slightly different scenarios. The methods used to investigate IRI in the past have ranged from tissue analysis to serum analysis of peripheral sampled blood or blood samples drawn in proximity to the liver. The lack of methods to investigate changes in the liver continuously inevitably leads to the sacrifice of a number of laboratory animals to make up for these shortcomings. In addition, clinical studies involving multiple biopsies from the liver are unethical and hazardous, and monitoring the changes using only blood tests is an inadequate method. Recent technical advances that may account for the shortcomings of the traditional methods described above include microdialysis, laser Doppler imaging, intravital microscopy and functional magnetic resonance imaging. Microdialysis offers a tool for investigating the changes that occur within the liver parenchyma in real time.

The method to counteract IRI when vascular occlusion is applied that has garnered the most support in the literature is the use of intermittent PM instead of continuous PM; however, the difference between these methods is small (94). Another available method that has been explored in the clinical setting is IPC, as listed in table 2. R-IPC is an exciting alternative to IPC that could reduce the inherent risk of the IPC procedure and shorten operation times. R-IPC has only been reported in one small clinical trial (251). Despite an increased interest in R-IPC and a number of experimental studies demonstrating a protective effect of R-IPC on IRI, R-IPC has not been compared directly to IPC. If preconditioning is to be used in the clinical setting, it is important to know how to apply it. Therefore, study I was

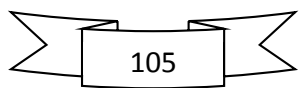


conducted to compare IPC and R-IPC. If $\mu \mathrm{D}$ is to be used in the clinic, it is advantageous to be able to use commercially available kits for the analysis; therefore, study I also included the hypothesis that $\mu \mathrm{D}$ could be performed exclusively with commercially available kits to monitor IRI in the liver.

IPC is more effective than R-IPC in reducing AST and ALT early (after 4 hour) in the reperfusion phase of IRI (figures 14 and 15, study I). Furthermore, animals treated with IPC exhibit fewer histological signs of IRI after IR than animals treated with R-IPC (figure 20). Similarly, less $\mu \mathrm{D}$ glycerol is detected after IR when animals are pre-treated with IPC than when R-IPC is applied as a pre-treatment (figure 19). The substances measured with $\mu \mathrm{D}$ in study I represent metabolic markers (glucose, pyruvate and lactate) and an injury marker (glycerol). The changes in these markers detected with $\mu \mathrm{D}$ were those expected with anaerobic metabolism with sufficient availability of glycogen (increased glucose and lactate) and cell damage (increased glycerol).

The increase in glucose noted with microdialysis in the IPC group prior to ischemia (figure 16) likely resulted from the preconditioning. This increase may be due to anaerobic metabolism already occurring during the short ischemia produced by preconditioning. However, glycolysis, which provides additional energy, is also a possible explanation for the increase. In a study using a porcine model, it was show that glycogen in the liver is depleted during ischemia, a finding that may support this explanation (268). On the other hand, the significant increase in lactate noted in the same segment (figure 18) supports the explanation that the preconditioning initiates anaerobic metabolism. The increase in glycerol noted at the same time further supports the notion that the preconditioning results in some degree of membrane disruption.

The protective effect of IPC in the clinical setting has largely been investigated with continuous PM. However, any difference between intermittent and continuous PM that might exist is to the advantage of intermittent PM. With this fact in mind and given the results indicating that a greater effect can be expected from IPC than R-IPC (study I), it seems reasonable to investigate the effect of IPC before intermittent PM in the clinical setting.

Although study II was somewhat underpowered, the study revealed no clinical advantages when IPC was added to intermittent PM. In the setting of minor liver resections (2-3 segments), the only difference between patients who received IPC and those who did not

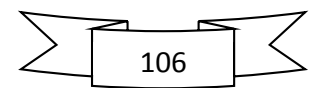


was in $\mu \mathrm{D}$ glucose on POD 2. In the subgroups undergoing major liver resections $(>3$ segments), a potentially beneficial effect of IPC was observed in laboratory values; lactate was reduced during the early post-operative phase in patients receiving IPC than in patients not receiving IPC (table 3). This finding was further supported by reduced $\mu \mathrm{D}$-glucose (figure 21 ), $\mu \mathrm{D}$-pyruvate (figure 22 ) and $\mu \mathrm{D}$-lactate (figure 23 ) immediately post-operative in the IPC group compared with the control group.

Although intermittent PM is recommended over continuous PM, the effectiveness of intermittent PM in the clinical setting in reducing IRI appears to be limited (94). The addition of IPC to intermittent PM is an insufficient means to counteract hepatic IRI (study II). In addition, it is somewhat cumbersome to apply IPC to all patients when PM is not always needed during liver resections. Our unit has changed the application of intermittent PM from routine application in a $15+5$ fashion to a more selective application for shorter periods of time when needed. This approach may decrease the total I/R. Furthermore, this approach is often applied in a manner that mimics IPC, making it important to pursue other means of protecting the liver against IRI when vascular occlusion is utilized during liver resections.

NO has been implicated in both IRI mechanisms and preconditioning and appears to be one of the key substances involved in these processes. iNOS rather than eNOS has previously been shown to be responsible for the majority of the NO production during acute inflammation. Therefore, iNOS may be implicated in IRI. Study III was designed to investigate the mechanisms involved in IPC and R-IPC with a special focus on NO involvement.

In study III, iNOS transcription was shown to be significantly increased in the R-IPC group compared with the IPC group (figure 26). Furthermore, the transcriptional activity in the R-IPC group was approximately identical to what was observed in the IRI group. This difference is likely a sign of greater inflammatory activity in the R-IPC group than in the IPC group. IL-1 receptor transcription was increased directly after ischemia in the IPC group compared with the R-IPC group (figure 27). The only difference in the treatment of the groups, and therefore the only plausible explanation for this difference, was how the preconditioning was applied. The initial ischemic insult to the liver (IPC) may have triggered the transcription of the IL-1 receptor, resulting in reduced IL-1 receptor transcription during reperfusion due to negative feedback. In study III, NOx was decreased during ischemia in both groups (figure 25). Until recently, NOx has been considered a product of the elimination

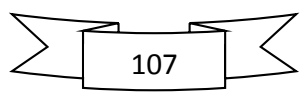


of NO and has thus been used as a surrogate marker for NO production. If this is the case, NOx would be expected to remain stable in ischemic tissue without any circulation. A possible explanation for the decrease in $\mu \mathrm{D}$-NOx that occurs during ischemia is the reduction of nitrite to NO (and nitrate to nitrite) (figure 10) that occurs primarily in acidic environments but also under physiological circumstances. A further explanation might be that NO can bind to other metabolic groups, such as thiols $(269,270)$. Despite the changes in parenchymal NOx detected with microdialysis, only minor changes were observed in the serum, indicating that serum measurements of NOx do not accurately reflect the situation in the liver parenchyma.

As NOx concentrations decreased in the liver parenchyma during ischemia (study III), it is possible that the administration of nitrite before the ischemic insult would decrease the IRI. When IRI is measured by serum AST and ALT levels 4 hours after 45-min segmental liver ischemia, the administration of nitrite before IR reduces IRI (figures 28 and 29, study IV). These findings are further supported by pathology findings demonstrating fewer signs of IRI in animals pre-treated with nitrite before IR than in non- treated animals (figure 36). Furthermore, lower parenchymal $(\mu \mathrm{D})$ glycerol levels accompany ischemia and early reperfusion when nitrite is administered before IR compared with no pre-treatment (figure 35), indicating less cell damage. This field has limited data; however, it has been shown in a mouse model that administrating nitrite before the onset of reperfusion (intraperitoneal injection) decreases both liver and heart IRI (271).It has been proposed that the protective effect of nitrite can be attributed to modifications of the mitochondrial electron transport chain that slow the ATP production as well as the production of ROS $(197,272)$. In study IV, $\mu$ Dglucose (figure 32) was found to increase in the control lobe directly after injection in the group treated with nitrite compared with untreated animals. At the same time, $\mu \mathrm{D}$-lactate (figure 34) was also increased. These changes, although subtle, might reflect the metabolic effects on the inhibition of the electron transport chain. Another possible explanation for the protective effect of nitrite administration is the inhibitory effect of nitrite on platelets (273). As demonstrated in figure 8, platelets play an active role in the swelling of the liver sinusoids that is central to the IRI. It is, however, worth noting that this effect also may increase the risk for bleeding, which represents the livery surgery complication that the use of PM aims to prevent, and IRI is the main drawback of PM. Thus, the development of a method to reduce IRI that increases the risk of bleeding might be challenging.

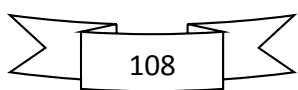


During liver resections, a low CVP is frequently maintained to reduce bleeding. One of the methods by which this effect is achieved is the administration of intravenous nitroglycerine. Nitroglycerine is a compound with a short half-life that is metabolized to nitrite in the liver (274). The studies performed on low CVP have largely focused on the effect of this method on bleeding and possible side effects related to systemic hypoperfusion. However, an additional effect might be buried within the administration of nitrite that can exert a protective effect against IRI. In a small randomized study where nitroglycerine was used to lower CVP, a tendency towards reduced ALT postoperatively among patients in the low CVP group was noted (275). These findings potentially reflect effects of nitroglycerine and nitrite that should be further explored in the clinical setting.

In summary, IPC as well as R-IPC exhibits some protective effects against liver IRI; however, the clinical effectiveness of these methods is questionable. Nitrite administered before $I / R$ also exerts a protective effect in these settings, and this finding should be further investigated in the clinical setting. Microdialysis appears to be a useful method to investigate liver IRI in experimental and clinical settings. 
$\sum[110]$ 


\section{Conclusions}

The main conclusions of this thesis are as follows:

IPC offers more protection against IRI than R-IPC when applied for the same amount of time.

$\mu \mathrm{D}$ with commercially available reagent kits can be used to monitor hepatic anaerobic metabolism and IRI in experimental and clinical settings.

The addition of IPC to intermittent PM does not offer clinically significant advantages compared with intermittent PM alone.

IPC decreases IRI-induced transcription of iNOS compared with R-IPC.

The administration of nitrite before liver ischemia reduces IRI. 
$\sum[12]$ 


\section{Future perspectives}

Currently, no secure means are available to avoid IRI when vascular occlusion is used to reduce bleeding during liver surgery. It appears unreasonable to believe that such a trauma to a well-vascularized organ can ever be completely avoided by any means. Therefore, the best method to avoid IRI should be to limit the use of vascular occlusion as much as possible. The use of the PM has changed from continuous to intermittent, and in our unit, intermittent PM is seldom applied for 15 minutes. With better anesthesiological methods as well as surgical instruments and techniques, the use of vascular occlusion can be minimized, thus decreasing the need for means to protect the liver against IRI. The more selective application of the PM also makes preconditioning less attractive given that the use of preconditioning could prove unnecessary in numerous cases.

From the technical aspect of the work presented in this thesis, it is clear that microdialysis can be applied in clinical and experimental liver surgeries, and this technique has great potential to reveal more about the mechanisms involved after liver resection. One interesting field of innovation that has become a clinical reality during the last two years is the ALPPS procedure. During this treatment, the patient is subjected to two liver operations over a period of 1-2 weeks. This timeframe with fast hypertrophy of the liver is an exciting field for research that could add to the general understanding of liver hypertrophy. Because of the short time span between the two procedures planned for this patient group, $\mu \mathrm{D}$ may serve as an optimal tool to investigate the causes of the hypertrophy.

Given that many liver surgery units prefer low CVP during liver surgery, which is often accomplished by treatment with intravenous nitroglycerine (glyceryl trinitrate), the results from study IV might become clinically relevant. Nitroglycerine exerts its vasodilatory effects via breakdown to NO, and NO rapidly oxidizes to nitrite. Thus, some of the beneficial effects of low CVP might be attributed to the use of nitroglycerine to achieve the low CVP. Fluid restriction is often used as the first method to maintain low CVP; we could compare the "classical approach" in patients who achieve the target CVP with a novel approach that omits this step and liberally applies nitroglycerine. In these settings, $\mu \mathrm{D}$ may be applied to assess the concentration of NOx in relation to nitroglycerine administration. The efficacy and safety (including bleeding) of nitrite should be further examined in animal studies and hopefully later in the clinical setting.

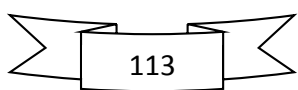


What really is needed though is the development of a surgical technique not requiring vascular occlusion. Inventing new equipment allowing transection of liver parenchyma without bleeding in spite of intact circulation to the liver could eliminate the risk of ischemic injury to the remnant liver parenchyma.

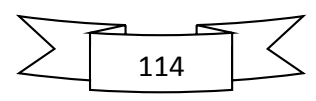




\section{Acknowledgments}

The research presented within this book is the work of many people in a research group and others to whom I owe my sincere gratitude. This list includes but is not limited to the following:

Per Sandström. Primary supervisor and good friend. For always finding time in your rather busy schedule to discuss research findings and future steps. You have provided me with the opportunity to combine the research with the clinic in a most rewarding way.

Per Gullstrand. Earlier primary supervisor, current co-supervisor and good friend. For your enthusiasm, encouragement and the ability to ask critical questions and never accept incomplete answers.

Tommy Sundqvist. Co-supervisor. For encouraging me to study the mechanisms involved in our results, for stimulating discussions and for the help you have provided in looking for explanations to our findings.

Anders Winbladh. Co-supervisor. For taking me into the world of liver IRI research without knowing me and for explaining many of the important steps involved. You have the ability to encourage and inspire your co-workers whenever needed.

Lena Trulsson. Earlier co-supervisor. For teaching me the fundamentals in laboratory science.

Linda Bojmar. Research engineer and fellow $\mathrm{PhD}$ student. For your patience and understanding and excellent work with the small samples provided to you. Good luck with your studies!

Hans Olsson. Co-author. Your pathology skills and critical thinking in the interpretation of the results have helped me better understand the limitations of my work.

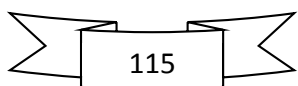


Rune Sjödahl. Professor of surgery, experienced clinical and academic surgeon. Your advice and ability to ask the right questions are always welcomed and can only increase the quality of the work.

The staff at the upper GI unit. Thank you for being supportive and generally being a pleasure to work with.

Other colleges at the department of surgery. You have put up with me on a daily basis in clinical work as well as during periods of more research-orientated endeavors. Your patience, understanding and encouragement mean a considerable amount to me.

The staff at the surgical ward and department of anesthesia and operation. Thanks for all of the help provided during the clinical study and for the positive collaboration in the clinical work.

The staff at the animal laboratory. You have been helpful and positive throughout the time I have spent at your workplace.

And last but certainly not least:

My family. Thank you for all your love, support, encouragement and understanding. You made this journey worthwhile! 


\section{References}

1. Homer. The Iliad, Book XX, translated by E.V.Rieu.: Penguin Books; 1950.

2. Macewen W. General Observations on the Introduction of Tracheal Tubes by the Mouth, Instead of Performing Tracheotomy or Laryngotomy. $\mathrm{Br}$ Med J. 1880;2(1021):122-4.

3. Shedden K. Cit Gurlt. Geschichte der Chir. 1898;3722.

4. Cerf C, Navasky V, editors. I wish I hadn't Said That: The Experts Speak and Get it Wrong. 1st ed: HarperCollins Publishers Ltd; 2000.

5. Lius A. Di un adenoma del fegato. Gazz Clini. 1886;23:225.

6. Lius A. Di un adenoma del fegato. Centralblatt Fur Chir. 1887/1886;5:99.

7. Langenbuch C. Ein Fall von Resecktion eines linksseitigen Schnurlappens der Leber. Berl Klin Wochenschr 1888;25.

8. Tiffany LM. Surgery of the Liver. Boston Med Surg J. 1890;122.

9. Lucke T. Entfernung des linken krebsigen Leberlappans. Zentralbl Chir. 1891;18:1.

10. Cantlie J. On a new arrangement of the right and left lobes of the liver. Proceedings Anatomical Society of Great Britain and Ireland. 1897(32):4-9.

11. Keen WW. Report of a case of resection of the liver for removal of hepatic tumours. Ann Surg. 1899;30:267-83.

12. Anschutz W. Uber die Resektion der Leber. Samml Klin-Vortr Chir. 1903(14):356.

13. Lin TYT, K. Mien, C. Chen, C. Study on lobectomy of the liver. J Formosa Med Assoc. 1958;57:742-9.

14. Jarnagin W. Blumgart's Surgery of the Liver, Biliary Tract, and Pancreas: 2-Volume Set, Expert Consult - Online and Print, 5e. 5th ed: Saunders Elsevier; 2012 04.05.2012. $1976 \mathrm{p}$.

15. Lin TY. Results in 107 hepatic lobectomies with a preliminary report on the use of a clamp to reduce blood loss. Ann Surg. 1973;177(4):413-21.

16. Pringle J. Notes on the Arrest of Hepatic Hemorrhage Due to Trauma. Ann Surg. 1908;48(4):541-9.

17. Richardson JD. Changes in the management of injuries to the liver and spleen. J Am Coll Surg. 2005;200(5):648-69.

18. Wendel W. Ueber Leberlappenresektion. Arch klin Chir. 1920(114):982-1000.

19. Dimick JB, Wainess RM, Cowan JA, Upchurch GR, Jr., Knol JA, Colletti LM. National trends in the use and outcomes of hepatic resection. J Am Coll Surg. 2004;199(1):31-8.

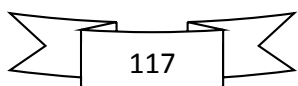


20. McColl RJ, You X, Ghali WA, Kaplan G, Myers R, Dixon E. Recent trends of hepatic resection in Canada: 1995-2004. J Gastrointest Surg. 2008;12(11):1839-46; discussion 46.

21. Cattell R. Successful removal of liver metastasis from carcinoma of the rectum. Lahey Clin Bull. 1940;2:7.

22. Lortat-Jacob JL, Robert HG. [Well defined technic for right hepatectomy]. Presse Med. 1952;60(26):549-51.

23. Lortat-Jacob JL, Robert HG, Henry C. [Case of right segmental hepatectomy]. Mem Acad Chir (Paris). 1952;78(8-9):244-51.

24. Lortat-Jacob JL, Robert HG, Henry C. [Excision of the right lobe of the liver for a malignant secondary tumor]. Arch Mal Appar Dig Mal Nutr. 1952;41(6):662-7.

25. Adam R, Miller R, Pitombo M, Wicherts DA, de Haas RJ, Bitsakou G, et al. Two-stage hepatectomy approach for initially unresectable colorectal hepatic metastases. Surg Oncol Clin N Am. 2007;16(3):525-36, viii.

26. Couinaud C, editor. Le Foie: Etudes Anatomiques et Chirurgicales. Paris: Masson; 1957.

27. Bismuth H. Surgical anatomy and anatomical surgery of the liver. World J Surg. 1982;6(1):3-9.

28. Quattlebaum JK. Massive resection of the liver. Ann Surg. 1953;137(6):787-96.

29. Starzl TE, Marchioro TL, Vonkaulla KN, Hermann G, Brittain RS, Waddell WR. Homotransplantation of the Liver in Humans. Surg Gynecol Obstet. 1963;117:659-76.

30. Fortner JG, Shiu MH, Kinne DW, Kim DK, Castro EB, Watson RC, et al. Major hepatic resection using vascular isolation and hypothermic perfusion. Ann Surg. 1974;180(4):644-52.

31. Pichlmayr R, Grosse H, Hauss J, Gubernatis G, Lamesch P, Bretschneider HJ. Technique and preliminary results of extracorporeal liver surgery (bench procedure) and of surgery on the in situ perfused liver. Br J Surg. 1990;77(1):21-6.

32. Stellamor K. [About the advantages of the sonographic diagnostic in the field of the radiologist (author's trans1)]. Rontgenblatter. 1976;29(11):544-52.

33. Bismuth H, Castaing D, Kunstlinger F. [Peroperative echography in hepatobiliary surgery]. Presse Med. 1984;13(30):1819-22.

34. Makuuchi M, Hasegawa H, Yamazaki S. Intraoperative ultrasonic examination for hepatectomy. Ultrasound Med Biol. 1983;Suppl 2:493-7.

35. Makuuchi M, Takayasu K, Takuma T, Yamazaki S, Hasegawa H, Nishiura S, et al. Preoperative transcatheter embolization of the portal branch for patients receiving extended lobectomy due to the bile duct carcinoma. J Jpn Soc Clin Surg. 1984;45:1420 .

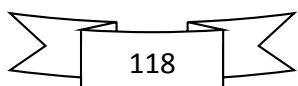


36. Nguyen KT, Gamblin TC, Geller DA. World review of laparoscopic liver resection2,804 patients. Ann Surg. 2009;250(5):831-41.

37. Schnitzbauer AA, Lang SA, Goessmann H, Nadalin S, Baumgart J, Farkas SA, et al. Right portal vein ligation combined with in situ splitting induces rapid left lateral liver lobe hypertrophy enabling 2-staged extended right hepatic resection in small-for-size settings. Ann Surg. 2012;255(3):405-14.

38. Adam R, Chiche L, Aloia T, Elias D, Salmon R, Rivoire M, et al. Hepatic resection for noncolorectal nonendocrine liver metastases: analysis of 1,452 patients and development of a prognostic model. Ann Surg. 2006;244(4):524-35.

39. Morris EJ, Forman D, Thomas JD, Quirke P, Taylor EF, Fairley L, et al. Surgical management and outcomes of colorectal cancer liver metastases. $\mathrm{Br} \mathrm{J}$ Surg. 2010;97(7):1110-8.

40. Abdalla EK. Resection of colorectal liver metastases. J Gastrointest Surg. 2011;15(3):416-9.

41. Nordlinger B, Rougier P. Liver metastases from colorectal cancer: the turning point. J Clin Oncol. 2002;20(6):1442-5.

42. Norstein J, Silen W. Natural history of liver metastases from colorectal carcinoma. J Gastrointest Surg. 1997;1(5):398-407.

43. Fortner JG, MacLean BJ, Kim DK, Howland WS, Turnbull AD, Goldiner P, et al. The seventies evolution in liver surgery for cancer. Cancer. 1981;47(9):2162-6.

44. Belghiti J, Hiramatsu K, Benoist S, Massault P, Sauvanet A, Farges O. Seven hundred forty-seven hepatectomies in the 1990s: an update to evaluate the actual risk of liver resection. J Am Coll Surg. 2000;191(1):38-46.

45. Taylor I. Colorectal liver metastases--to treat or not to treat? Br J Surg. 1985;72(7):5116.

46. Bismuth H, Adam R, Levi F, Farabos C, Waechter F, Castaing D, et al. Resection of nonresectable liver metastases from colorectal cancer after neoadjuvant chemotherapy. Ann Surg. 1996;224(4):509-20; discussion 20-2.

47. Adam R, Wicherts DA, de Haas RJ, Ciacio O, Levi F, Paule B, et al. Patients with initially unresectable colorectal liver metastases: is there a possibility of cure? J Clin Oncol. 2009;27(11):1829-35.

48. Nordlinger B, Sorbye H, Glimelius B, Poston GJ, Schlag PM, Rougier P, et al. Perioperative chemotherapy with FOLFOX4 and surgery versus surgery alone for resectable liver metastases from colorectal cancer (EORTC Intergroup trial 40983): a randomised controlled trial. Lancet. 2008;371(9617):1007-16.

49. Rees M, Plant G, Wells J, Bygrave S. One hundred and fifty hepatic resections: evolution of technique towards bloodless surgery. Br J Surg. 1996;83(11):1526-9.

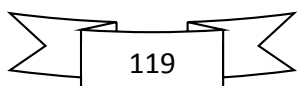


50. van den Broek MA, van Dam RM, Malago M, Dejong CH, van Breukelen GJ, Olde Damink SW. Feasibility of randomized controlled trials in liver surgery using surgeryrelated mortality or morbidity as endpoint. Br J Surg. 2009;96(9):1005-14.

51. Kopetz S, Chang GJ, Overman MJ, Eng C, Sargent DJ, Larson DW, et al. Improved survival in metastatic colorectal cancer is associated with adoption of hepatic resection and improved chemotherapy. J Clin Oncol. 2009;27(22):3677-83.

52. Skandalakis JE, Skandalakis LJ, Skandalakis PN, Mirilas P. Hepatic surgical anatomy. Surg Clin North Am. 2004;84(2):413-35, viii.

53. Rouviere H. Sur la configuration et la signification du sillon du processus caude. Bulletins et memoires de la societe anatomique de paris. 1924;94:355-8.

54. McIndoe A, Counseller V. The bilaterality of the liver. Arch Surg. 1927;15(4):589-612.

55. Hjortsjo $\mathrm{CH}$. The topography of the intrahepatic duct systems. Acta Anat (Basel). 1951;11(4):599-615.

56. Healey JE, Jr., Schroy PC. Anatomy of the biliary ducts within the human liver; analysis of the prevailing pattern of branchings and the major variations of the biliary ducts. AMA Arch Surg. 1953;66(5):599-616.

57. Goldsmith NA, Woodburne RT. The surgical anatomy pertaining to liver resection. Surg Gynecol Obstet. 1957;105(3):310-8.

58. Strasberg SM. Terminology of liver anatomy and liver resections: coming to grips with hepatic Babel. J Am Coll Surg. 1997;184(4):413-34.

59. Strasberg SM. Nomenclature of hepatic anatomy and resections: a review of the Brisbane 2000 system. J Hepatobiliary Pancreat Surg. 2005;12(5):351-5.

60. Strasberg SM, Phillips C. Use and dissemination of the brisbane 2000 nomenclature of liver anatomy and resections. Ann Surg. 2013;257(3):377-82.

61. Association TCotIH-P-B. The Brisbane 2000 Terminology of Liver Anatomy and Resections. HPB 2000;2(3):333-9.

62. Pamecha V, Gurusamy KS, Sharma D, Davidson BR. Techniques for liver parenchymal transection: a meta-analysis of randomized controlled trials. HPB (Oxford). 2009;11(4):275-81.

63. Hodgson WJ. Ultrasonic surgery. Ann R Coll Surg Engl. 1980;62(6):459-61.

64. Gurusamy KS, Pamecha V, Sharma D, Davidson BR. Techniques for liver parenchymal transection in liver resection. Cochrane Database Syst Rev. 2009(1):CD006880.

65. Fan ST, Lai EC, Lo CM, Chu KM, Liu CL, Wong J. Hepatectomy with an ultrasonic dissector for hepatocellular carcinoma. Br J Surg. 1996;83(1):117-20.

66. Lesurtel M, Selzner M, Petrowsky H, McCormack L, Clavien PA. How should transection of the liver be performed?: a prospective randomized study in 100 consecutive patients: comparing four different transection strategies. Ann Surg. 2005;242(6):814-22, discussion 22-3.

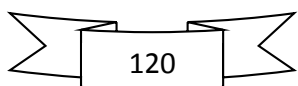


67. Koo BN, Kil HK, Choi JS, Kim JY, Chun DH, Hong YW. Hepatic resection by the Cavitron Ultrasonic Surgical Aspirator increases the incidence and severity of venous air embolism. Anesth Analg. 2005;101(4):966-70, table of contents.

68. Schmidbauer S, Hallfeldt KK, Sitzmann G, Kantelhardt T, Trupka A. Experience with ultrasound scissors and blades (UltraCision) in open and laparoscopic liver resection. Ann Surg. 2002;235(1):27-30.

69. Takayama T, Makuuchi M, Kubota K, Harihara Y, Hui AM, Sano K, et al. Randomized comparison of ultrasonic vs clamp transection of the liver. Arch Surg. 2001;136(8):9228.

70. Horgan PG. A novel technique for parenchymal division during hepatectomy. Am J Surg. 2001;181(3):236-7.

71. Doklestic K, Karamarkovic A, Stefanovic B, Milic N, Gregoric P, Djukic V, et al. The efficacy of three transection techniques of the liver resection: a randomized clinical trial. Hepatogastroenterology. 2012;59(117):1501-6.

72. Saiura A, Yamamoto J, Koga R, Sakamoto Y, Kokudo N, Seki M, et al. Usefulness of LigaSure for liver resection: analysis by randomized clinical trial. Am J Surg. 2006;192(1):41-5.

73. Slakey DP. Laparoscopic liver resection using a bipolar vessel-sealing device: LigaSure. HPB (Oxford). 2008;10(4):253-5.

74. Geller DA, Tsung A, Maheshwari V, Rutstein LA, Fung JJ, Marsh JW. Hepatic resection in 170 patients using saline-cooled radiofrequency coagulation. HPB (Oxford). 2005;7(3):208-13.

75. Zhang S, Zheng Y, Wu B, Ji S, Yu Z, Zhang Q. Is the TissueLink dissecting sealer a better liver resection device than clamp-crushing? A meta-analysis and system review. Hepatogastroenterology. 2012;59(120):2602-8.

76. Weber JC, Navarra G, Jiao LR, Nicholls JP, Jensen SL, Habib NA. New technique for liver resection using heat coagulative necrosis. Ann Surg. 2002;236(5):560-3.

77. Delis SG, Madariaga J, Bakoyiannis A, Dervenis C. Current role of bloodless liver resection. World J Gastroenterol. 2007;13(6):826-9.

78. Rau HG, Duessel AP, Wurzbacher S. The use of water-jet dissection in open and laparoscopic liver resection. HPB (Oxford). 2008;10(4):275-80.

79. D'Amico FE, Allen PJ, Eaton AA, DeMatteo RP, Fong Y, Kingham TP, et al. Vascular inflow control during hemi-hepatectomy: a comparison between intrahepatic pedicle ligation and extrahepatic vascular ligation. HPB (Oxford). 2013;15(6):449-56.

80. Cresswell AB, Welsh FK, John TG, Rees M. Evaluation of intrahepatic, extraGlissonian stapling of the right porta hepatis vs. classical extrahepatic dissection during right hepatectomy. HPB (Oxford). 2009;11(6):493-8.

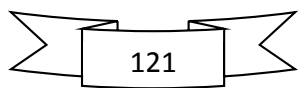


81. Habib M, Cresswell AB, Chandrakumaran K, Welsh FK, John TG, Rees M. Extrahepatic versus intrahepatic hilar control for right hepatectomy: an updated experience. Dig Surg. 2012;29(1):18-22.

82. Elias D, Cavalcanti A, Sabourin JC, Lassau N, Pignon JP, Ducreux M, et al. Resection of liver metastases from colorectal cancer: the real impact of the surgical margin. Eur $\mathrm{J}$ Surg Oncol. 1998;24(3):174-9.

83. Elias D, Cavalcanti A, Sabourin JC, Pignon JP, Ducreux M, Lasser P. Results of 136 curative hepatectomies with a safety margin of less than $10 \mathrm{~mm}$ for colorectal metastases. J Surg Oncol. 1998;69(2):88-93.

84. Kokudo N, Miki Y, Sugai S, Yanagisawa A, Kato Y, Sakamoto Y, et al. Genetic and histological assessment of surgical margins in resected liver metastases from colorectal carcinoma: minimum surgical margins for successful resection. Arch Surg. 2002;137(7):833-40.

85. Bodingbauer M, Tamandl D, Schmid K, Plank C, Schima W, Gruenberger T. Size of surgical margin does not influence recurrence rates after curative liver resection for colorectal cancer liver metastases. Br J Surg. 2007;94(9):1133-8.

86. Shi M, Zhang CQ, Zhang YQ, Liang XM, Li JQ. Micrometastases of solitary hepatocellular carcinoma and appropriate resection margin. World $\mathrm{J}$ Surg. 2004;28(4):376-81.

87. Shi M, Guo RP, Lin XJ, Zhang YQ, Chen MS, Zhang CQ, et al. Partial hepatectomy with wide versus narrow resection margin for solitary hepatocellular carcinoma: a prospective randomized trial. Ann Surg. 2007;245(1):36-43.

88. DeMatteo RP, Palese C, Jarnagin WR, Sun RL, Blumgart LH, Fong Y. Anatomic segmental hepatic resection is superior to wedge resection as an oncologic operation for colorectal liver metastases. J Gastrointest Surg. 2000;4(2):178-84.

89. Zorzi D, Mullen JT, Abdalla EK, Pawlik TM, Andres A, Muratore A, et al. Comparison between hepatic wedge resection and anatomic resection for colorectal liver metastases. J Gastrointest Surg. 2006;10(1):86-94.

90. Poon RT, Fan ST, Lo CM, Liu CL, Lam CM, Yuen WK, et al. Improving perioperative outcome expands the role of hepatectomy in management of benign and malignant hepatobiliary diseases: analysis of 1222 consecutive patients from a prospective database. Ann Surg. 2004;240(4):698-708; discussion -10.

91. Adam R, Frilling A, Elias D, Laurent C, Ramos E, Capussotti L, et al. Liver resection of colorectal metastases in elderly patients. Br J Surg. 2010;97(3):366-76.

92. Kooby DA, Stockman J, Ben-Porat L, Gonen M, Jarnagin WR, Dematteo RP, et al. Influence of transfusions on perioperative and long-term outcome in patients following hepatic resection for colorectal metastases. Ann Surg. 2003;237(6):860-9; discussion 970.

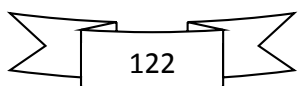


93. Hoti E, Salloum C, Azoulay D. Hepatic resection with in situ hypothermic perfusion is superior to other resection techniques. Dig Surg. 2011;28(2):94-9.

94. Gurusamy KS, Sheth H, Kumar Y, Sharma D, Davidson BR. Methods of vascular occlusion for elective liver resections. Cochrane Database Syst Rev. 2009(1):CD007632.

95. Belghiti J, Noun R, Zante E, Ballet T, Sauvanet A. Portal triad clamping or hepatic vascular exclusion for major liver resection. A controlled study. Ann Surg. 1996;224(2):155-61.

96. Rahbari NN, Koch M, Mehrabi A, Weidmann K, Motschall E, Kahlert C, et al. Portal triad clamping versus vascular exclusion for vascular control during hepatic resection: a systematic review and meta-analysis. J Gastrointest Surg. 2009;13(3):558-68.

97. Elias D, Lasser P, Debaene B, Doidy L, Billard V, Spencer A, et al. Intermittent vascular exclusion of the liver (without vena cava clamping) during major hepatectomy. Br J Surg. 1995;82(11):1535-9.

98. Smyrniotis VE, Kostopanagiotou GG, Gamaletsos EL, Vassiliou JG, Voros DC, Fotopoulos AC, et al. Total versus selective hepatic vascular exclusion in major liver resections. Am J Surg. 2002;183(2):173-8.

99. Zhou W, Li A, Pan Z, Fu S, Yang Y, Tang L, et al. Selective hepatic vascular exclusion and Pringle maneuver: a comparative study in liver resection. Eur J Surg Oncol. 2008;34(1):49-54.

100. Jones RM, Moulton CE, Hardy KJ. Central venous pressure and its effect on blood loss during liver resection. Br J Surg. 1998;85(8):1058-60.

101. Melendez JA, Arslan V, Fischer ME, Wuest D, Jarnagin WR, Fong Y, et al. Perioperative Outcomes of Major Hepatic Resections under Low Central Venous Pressure Anesthesia: Blood Loss, Blood Transfusion, and the Risk of Postoperative Renal Dysfunction. J Am Coll Surg. 1998;187(6):620-5.

102. Gurusamy KS, Li J, Vaughan J, Sharma D, Davidson BR. Cardiopulmonary interventions to decrease blood loss and blood transfusion requirements for liver resection. Cochrane Database Syst Rev. 2012;5:CD007338.

103. Steele G, Jr., Ravikumar TS. Resection of hepatic metastases from colorectal cancer. Biologic perspective. Ann Surg. 1989;210(2):127-38.

104. de Haas RJ, Wicherts DA, Adam R. Resection of colorectal liver metastases with extrahepatic disease. Dig Surg. 2008;25(6):461-6.

105. Ribero D, Chun YS, Vauthey JN. Standardized liver volumetry for portal vein embolization. Semin Intervent Radiol. 2008;25(2):104-9.

106. Vauthey JN, Pawlik TM, Ribero D, Wu TT, Zorzi D, Hoff PM, et al. Chemotherapy regimen predicts steatohepatitis and an increase in 90-day mortality after surgery for hepatic colorectal metastases. J Clin Oncol. 2006;24(13):2065-72.

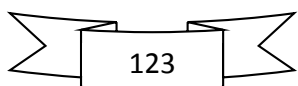


107. Abdalla EK, Adam R, Bilchik AJ, Jaeck D, Vauthey JN, Mahvi D. Improving resectability of hepatic colorectal metastases: expert consensus statement. Ann Surg Oncol. 2006;13(10):1271-80.

108. Adam R, Bismuth H, Castaing D, Waechter F, Navarro F, Abascal A, et al. Repeat hepatectomy for colorectal liver metastases. Ann Surg. 1997;225(1):51-60; discussion 2 .

109. Adam R, Pascal G, Azoulay D, Tanaka K, Castaing D, Bismuth H. Liver resection for colorectal metastases: the third hepatectomy. Ann Surg. 2003;238(6):871-83; discussion 83-4.

110. Antoniou A, Lovegrove RE, Tilney HS, Heriot AG, John TG, Rees M, et al. Metaanalysis of clinical outcome after first and second liver resection for colorectal metastases. Surgery. 2007;141(1):9-18.

111. Rhodes RS, DePalma RG, Druet RL. Reversibility of ischemically induced mitochondrial dysfunction with reperfusion. Surg Gynecol Obstet. 1977;145(5):719-24.

112. Silver EH, Szabo S. Role of lipid peroxidation in tissue injury after hepatic ischemia. Exp Mol Pathol. 1983;38(1):69-76.

113. Toledo-Pereyra LH, Simmons RL, Najarian JS. Factors determining successful liver preservation for transplantation. Ann Surg. 1975;181(3):289-98.

114. Jolly PC, Foster JH. Hepatic inflow stasis. Surgery. 1963;54:45-55.

115. Lentsch AB, Kato A, Yoshidome H, McMasters KM, Edwards MJ. Inflammatory mechanisms and therapeutic strategies for warm hepatic ischemia/reperfusion injury. Hepatology. 2000;32(2):169-73.

116. Teoh NC, Farrell GC. Hepatic ischemia reperfusion injury: pathogenic mechanisms and basis for hepatoprotection. J Gastroenterol Hepatol. 2003;18(8):891-902.

117. Selzner M, Selzner N, Jochum W, Graf R, Clavien PA. Increased ischemic injury in old mouse liver: an ATP-dependent mechanism. Liver Transpl. 2007;13(3):382-90.

118. Gasbarrini A, Borle AB, Farghali H, Bender C, Francavilla A, Van Thiel D. Effect of anoxia on intracellular ATP, $\mathrm{Na}+\mathrm{i}, \mathrm{Ca} 2+\mathrm{i}, \mathrm{Mg} 2+\mathrm{i}$, and cytotoxicity in rat hepatocytes. $\mathrm{J}$ Biol Chem. 1992;267(10):6654-63.

119. Bronk SF, Gores GJ. Efflux of protons from acidic vesicles contributes to cytosolic acidification of hepatocytes during ATP depletion. Hepatology. 1991;14(4 Pt 1):626-33.

120. Nowak G, Ungerstedt J, Wernerson A, Ungerstedt U, Ericzon BG. Hepatic cell membrane damage during cold preservation sensitizes liver grafts to rewarming injury. $\mathrm{J}$ Hepatobiliary Pancreat Surg. 2003;10(3):200-5.

121. Jaeschke H. Mechanisms of reperfusion injury after warm ischemia of the liver. $J$ Hepatobiliary Pancreat Surg. 1998;5(4):402-8.

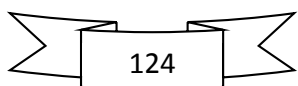


122. Engerson TD, McKelvey TG, Rhyne DB, Boggio EB, Snyder SJ, Jones HP. Conversion of xanthine dehydrogenase to oxidase in ischemic rat tissues. $\mathrm{J}$ Clin Invest. 1987;79(6):1564-70.

123. Jaeschke H, Smith CV, Mitchell JR. Reactive oxygen species during ischemia-reflow injury in isolated perfused rat liver. J Clin Invest. 1988;81(4):1240-6.

124. Jeon BR, Yeom DH, Lee SM. Protective effect of allopurinol on hepatic energy metabolism in ischemic and reperfused rat liver. Shock. 2001;15(2):112-7.

125. Jaeschke H, Mitchell JR. Mitochondria and xanthine oxidase both generate reactive oxygen species in isolated perfused rat liver after hypoxic injury. Biochem Biophys Res Commun. 1989;160(1):140-7.

126. Zhou W, Zhang Y, Hosch MS, Lang A, Zwacka RM, Engelhardt JF. Subcellular site of superoxide dismutase expression differentially controls AP-1 activity and injury in mouse liver following ischemia/reperfusion. Hepatology. 2001;33(4):902-14.

127. Mari M, Morales A, Colell A, Garcia-Ruiz C, Fernandez-Checa JC. Mitochondrial glutathione, a key survival antioxidant. Antioxid Redox Signal. 2009;11(11):2685-700.

128. Babior BM, Lambeth JD, Nauseef W. The neutrophil NADPH oxidase. Archives of biochemistry and biophysics. 2002;397(2):342-4.

129. Jaquet V, Scapozza L, Clark RA, Krause KH, Lambeth JD. Small-molecule NOX inhibitors: ROS-generating NADPH oxidases as therapeutic targets. Antioxid Redox Signal. 2009;11(10):2535-52.

130. Jaeschke H. Molecular mechanisms of hepatic ischemia-reperfusion injury and preconditioning. Am J Physiol Gastrointest Liver Physiol. 2003;284(1):G15-26.

131. Liu PG, He SQ, Zhang YH, Wu J. Protective effects of apocynin and allopurinol on ischemia/reperfusion-induced liver injury in mice. World $\mathrm{J}$ Gastroenterol. 2008;14(18):2832-7.

132. Lentsch AB, Yoshidome H, Kato A, Warner RL, Cheadle WG, Ward PA, et al. Requirement for interleukin-12 in the pathogenesis of warm hepatic ischemia/reperfusion injury in mice. Hepatology. 1999;30(6):1448-53.

133. Diepenhorst GM, van Gulik TM, Hack CE. Complement-mediated ischemiareperfusion injury: lessons learned from animal and clinical studies. Ann Surg. 2009;249(6):889-99.

134. Jaeschke H, Farhood A. Neutrophil and Kupffer cell-induced oxidant stress and ischemia-reperfusion injury in rat liver. Am J Physiol. 1991;260(3 Pt 1):G355-62.

135. Wanner GA, Ertel W, Muller P, Hofer Y, Leiderer R, Menger MD, et al. Liver ischemia and reperfusion induces a systemic inflammatory response through Kupffer cell activation. Shock. 1996;5(1):34-40.

136. Shito M, Wakabayashi G, Ueda M, Shimazu M, Shirasugi N, Endo M, et al. Interleukin 1 receptor blockade reduces tumor necrosis factor production, tissue injury, and

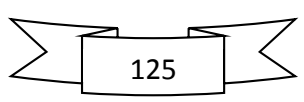


mortality after hepatic ischemia-reperfusion in the rat. Transplantation. 1997;63(1):1438.

137. Colletti LM, Remick DG, Burtch GD, Kunkel SL, Strieter RM, Campbell DA, Jr. Role of tumor necrosis factor-alpha in the pathophysiologic alterations after hepatic ischemia/reperfusion injury in the rat. J Clin Invest. 1990;85(6):1936-43.

138. Suzuki S, Toledo-Pereyra LH. Interleukin 1 and tumor necrosis factor production as the initial stimulants of liver ischemia and reperfusion injury. J Surg Res. 1994;57(2):253-8.

139. Camargo CA, Jr., Madden JF, Gao W, Selvan RS, Clavien PA. Interleukin-6 protects liver against warm ischemia/reperfusion injury and promotes hepatocyte proliferation in the rodent. Hepatology. 1997;26(6):1513-20.

140. Jaeschke H, Farhood A, Bautista AP, Spolarics Z, Spitzer JJ. Complement activates Kupffer cells and neutrophils during reperfusion after hepatic ischemia. Am J Physiol. 1993;264(4 Pt 1):G801-9.

141. Lehmann TG, Heger M, Munch S, Kirschfink M, Klar E. In vivo microscopy reveals that complement inhibition by $\mathrm{C} 1$-esterase inhibitor reduces ischemia/reperfusion injury in the liver. Transpl Int. 2000;13 Suppl 1:S547-50.

142. Lehmann TG, Koeppel TA, Munch S, Heger M, Kirschfink M, Klar E, et al. Impact of inhibition of complement by sCR1 on hepatic microcirculation after warm ischemia. Microvasc Res. 2001;62(3):284-92.

143. Arumugam TV, Woodruff TM, Stocks SZ, Proctor LM, Pollitt S, Shiels IA, et al. Protective effect of a human $\mathrm{C} 5 \mathrm{a}$ receptor antagonist against hepatic ischaemiareperfusion injury in rats. J Hepatol. 2004;40(6):934-41.

144. Heijnen BH, Straatsburg IH, Padilla ND, Van Mierlo GJ, Hack CE, Van Gulik TM. Inhibition of classical complement activation attenuates liver ischaemia and reperfusion injury in a rat model. Clin Exp Immunol. 2006;143(1):15-23.

145. Hanschen M, Zahler S, Krombach F, Khandoga A. Reciprocal activation between CD4+ $\mathrm{T}$ cells and Kupffer cells during hepatic ischemia-reperfusion. Transplantation. 2008;86(5):710-8.

146. Zwacka RM, Zhang Y, Halldorson J, Schlossberg H, Dudus L, Engelhardt JF. CD4(+) T-lymphocytes mediate ischemia/reperfusion-induced inflammatory responses in mouse liver. J Clin Invest. 1997;100(2):279-89.

147. Caldwell CC, Tschoep J, Lentsch AB. Lymphocyte function during hepatic ischemia/reperfusion injury. J Leukoc Biol. 2007;82(3):457-64.

148. Jaeschke H, Bautista AP, Spolarics Z, Spitzer JJ. Superoxide generation by Kupffer cells and priming of neutrophils during reperfusion after hepatic ischemia. Free Radic Res Commun. 1991;15(5):277-84.

149. Colletti LM, Cortis A, Lukacs N, Kunkel SL, Green M, Strieter RM. Tumor necrosis factor up-regulates intercellular adhesion molecule 1 , which is important in the

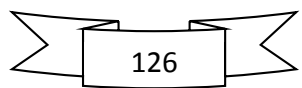


neutrophil-dependent lung and liver injury associated with hepatic ischemia and reperfusion in the rat. Shock. 1998;10(3):182-91.

150. Ley K, Laudanna C, Cybulsky MI, Nourshargh S. Getting to the site of inflammation: the leukocyte adhesion cascade updated. Nat Rev Immunol. 2007;7(9):678-89.

151. Cutrn JC, Perrelli MG, Cavalieri B, Peralta C, Rosell Catafau J, Poli G. Microvascular dysfunction induced by reperfusion injury and protective effect of ischemic preconditioning. Free Radic Biol Med. 2002;33(9):1200-8.

152. Wong J, Johnston B, Lee SS, Bullard DC, Smith CW, Beaudet AL, et al. A minimal role for selectins in the recruitment of leukocytes into the inflamed liver microvasculature. J Clin Invest. 1997;99(11):2782-90.

153. El-Benna J, Dang PM, Gougerot-Pocidalo MA. Priming of the neutrophil NADPH oxidase activation: role of p47phox phosphorylation and NOX2 mobilization to the plasma membrane. Semin Immunopathol. 2008;30(3):279-89.

154. Jaeschke H, Bautista AP, Spolarics Z, Spitzer JJ. Superoxide generation by neutrophils and Kupffer cells during in vivo reperfusion after hepatic ischemia in rats. J Leukoc Biol. 1992;52(4):377-82.

155. Jaeschke H, Smith CW. Mechanisms of neutrophil-induced parenchymal cell injury. J Leukoc Biol. 1997;61(6):647-53.

156. Nieminen AL, Saylor AK, Tesfai SA, Herman B, Lemasters JJ. Contribution of the mitochondrial permeability transition to lethal injury after exposure of hepatocytes to tbutylhydroperoxide. Biochem J. 1995;307 ( Pt 1):99-106.

157. Jaeschke H. Mechanisms of Liver Injury. II. Mechanisms of neutrophil-induced liver cell injury during hepatic ischemia-reperfusion and other acute inflammatory conditions. Am J Physiol Gastrointest Liver Physiol. 2006;290(6):G1083-8.

158. Ho JS, Buchweitz JP, Roth RA, Ganey PE. Identification of factors from rat neutrophils responsible for cytotoxicity to isolated hepatocytes. J Leukoc Biol. 1996;59(5):716-24.

159. Chosay JG, Essani NA, Dunn CJ, Jaeschke H. Neutrophil margination and extravasation in sinusoids and venules of liver during endotoxin-induced injury. Am J Physiol. 1997;272(5 Pt 1):G1195-200.

160. Vollmar B, Glasz J, Leiderer R, Post S, Menger MD. Hepatic microcirculatory perfusion failure is a determinant of liver dysfunction in warm ischemia-reperfusion. Am J Pathol. 1994;145(6):1421-31.

161. Khandoga A, Biberthaler P, Messmer K, Krombach F. Platelet-endothelial cell interactions during hepatic ischemia-reperfusion in vivo: a systematic analysis. Microvasc Res. 2003;65(2):71-7.

162. Nakano Y, Kondo T, Matsuo R, Hashimoto I, Kawasaki T, Kohno K, et al. Platelet dynamics in the early phase of postischemic liver in vivo. J Surg Res. 2008;149(2):1928 .

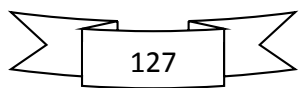


163. Peralta C, Closa D, Hotter G, Gelpi E, Prats N, Rosello-Catafau J. Liver ischemic preconditioning is mediated by the inhibitory action of nitric oxide on endothelin. Biochem Biophys Res Commun. 1996;229(1):264-70.

164. Ku Y, Kusunoki N, Shiotani M, Maeda I, Iwasaki T, Tominaga M, et al. Stimulation of haematogenous liver metastases by ischaemia-reperfusion in rats. Eur J Surg. 1999; 165(8):801-7.

165. Doi K, Horiuchi T, Uchinami M, Tabo T, Kimura N, Yokomachi J, et al. Hepatic ischemia-reperfusion promotes liver metastasis of colon cancer. J Surg Res. 2002;105(2):243-7.

166. van der Bilt JD, Kranenburg O, Verheem A, van Hillegersberg R, Borel Rinkes IH. Selective portal clamping to minimize hepatic ischaemia-reperfusion damage and avoid accelerated outgrowth of experimental colorectal liver metastases. $\mathrm{Br} \mathrm{J}$ Surg. 2006;93(8):1015-22.

167. McNaughton L, Puttagunta L, Martinez-Cuesta MA, Kneteman N, Mayers I, Moqbel R, et al. Distribution of nitric oxide synthase in normal and cirrhotic human liver. Proc Natl Acad Sci U S A. 2002;99(26):17161-6.

168. Koti RS, Tsui J, Lobos E, Yang W, Seifalian AM, Davidson BR. Nitric oxide synthase distribution and expression with ischemic preconditioning of the rat liver. FASEB J. 2005;19(9):1155-7.

169. Koeppel TA, Mihaljevic N, Kraenzlin B, Loehr M, Jesenofsky R, Post S, et al. Enhanced iNOS gene expression in the steatotic rat liver after normothermic ischemia. Eur Surg Res. 2007;39(5):303-11.

170. Amersi F, Shen XD, Moore C, Melinek J, Busuttil RW, Kupiec-Weglinski JW, et al. Fibronectin-alpha 4 beta 1 integrin-mediated blockade protects genetically fat Zucker rat livers from ischemia/reperfusion injury. Am J Pathol. 2003;162(4):1229-39.

171. Kimura H, Katsuramaki T, Isobe M, Nagayama M, Meguro M, Kukita K, et al. Role of inducible nitric oxide synthase in pig liver transplantation. J Surg Res. 2003;111(1):2837.

172. Hines IN, Harada H, Flores S, Gao B, McCord JM, Grisham MB. Endothelial nitric oxide synthase protects the post-ischemic liver: potential interactions with superoxide. Biomed Pharmacother. 2005;59(4):183-9.

173. Peralta C, Hotter G, Closa D, Prats N, Xaus C, Gelpi E, et al. The protective role of adenosine in inducing nitric oxide synthesis in rat liver ischemia preconditioning is mediated by activation of adenosine A2 receptors. Hepatology. 1999;29(1):126-32.

174. Uhlmann D, Gaebel G, Armann B, Ludwig S, Hess J, Pietsch UC, et al. Attenuation of proinflammatory gene expression and microcirculatory disturbances by endothelin A receptor blockade after orthotopic liver transplantation in pigs. Surgery. 2006;139(1):61-72.

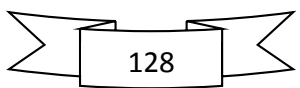


175. Farmer DG, Kaldas F, Anselmo D, Katori M, Shen XD, Lassman C, et al. Tezosentan, a novel endothelin receptor antagonist, markedly reduces rat hepatic ischemia and reperfusion injury in three different models. Liver Transpl. 2008;14(12):1737-44.

176. Gustafsson BI, Wallin M, Delbro DS, Friman S. Nitric oxide-mediated effects on liver blood flow. Transplant Proc. 2005;37(8):3338-9.

177. Koken T, Inal M. The effect of nitric oxide on ischemia-reperfusion injury in rat liver. Clin Chim Acta. 1999;288(1-2):55-62.

178. Duranski MR, Elrod JW, Calvert JW, Bryan NS, Feelisch M, Lefer DJ. Genetic overexpression of eNOS attenuates hepatic ischemia-reperfusion injury. Am J Physiol Heart Circ Physiol. 2006;291(6):H2980-6.

179. Nilsson B, Yoshida T, Delbro D, Andius S, Friman S. Pretreatment with L-arginine reduces ischemia/reperfusion injury of the liver. Transplant Proc. 1997;29(7):3111-2.

180. Cottart CH, Do L, Blanc MC, Vaubourdolle M, Descamps G, Durand D, et al. Hepatoprotective effect of endogenous nitric oxide during ischemia-reperfusion in the rat. Hepatology. 1999;29(3):809-13.

181. Nilsson B, Delbro D, Wallin M, Friman S. Protective effect of nitric oxide and prostaglandin $\mathrm{E}(2)$ in ischemia/reperfusion injury of the liver. Transplant Proc. 2001;33(4):2518-20.

182. Kobayashi H, Nonami T, Kurokawa T, Takeuchi Y, Harada A, Nakao A, et al. Role of endogenous nitric oxide in ischemia-reperfusion injury in rat liver. J Surg Res. 1995;59(6):772-9.

183. Koti RS, Yang W, Dashwood MR, Davidson BR, Seifalian AM. Effect of ischemic preconditioning on hepatic microcirculation and function in a rat model of ischemia reperfusion injury. Liver Transpl. 2002;8(12):1182-91.

184. Caban A, Oczkowicz G, Abdel-Samad O, Cierpka L. Influence of ischemic preconditioning and nitric oxide on microcirculation and the degree of rat liver injury in the model of ischemia and reperfusion. Transplant Proc. 2006;38(1):196-8.

185. Isobe M, Katsuramaki T, Kimura H, Nagayama M, Matsuno T, Yagihashi A, et al. Role of inducible nitric oxide synthase on hepatic ischemia and reperfusion injury. Transplant Proc. 2000;32(7):1650-2.

186. Takamatsu Y, Shimada K, Yamaguchi K, Kuroki S, Chijiiwa K, Tanaka M. Inhibition of inducible nitric oxide synthase prevents hepatic, but not pulmonary, injury following ischemia-reperfusion of rat liver. Dig Dis Sci. 2006;51(3):571-9.

187. Meguro M, Katsuramaki T, Nagayama M, Kimura H, Isobe M, Kimura Y, et al. A novel inhibitor of inducible nitric oxide synthase (ONO-1714) prevents critical warm ischemia-reperfusion injury in the pig liver. Transplantation. 2002;73(9):1439-46.

188. Tsuchihashi S, Kaldas F, Chida N, Sudo Y, Tamura K, Zhai Y, et al. FK330, a novel inducible nitric oxide synthase inhibitor, prevents ischemia and reperfusion injury in rat liver transplantation. Am J Transplant. 2006;6(9):2013-22.

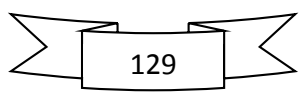


189. Bartosz G. Peroxynitrite: mediator of the toxic action of nitric oxide. Acta Biochim Pol. 1996;43(4):645-59.

190. Lee VG, Johnson ML, Baust J, Laubach VE, Watkins SC, Billiar TR. The roles of iNOS in liver ischemia-reperfusion injury. Shock. 2001;16(5):355-60.

191. Kawachi S, Hines IN, Laroux FS, Hoffman J, Bharwani S, Gray L, et al. Nitric oxide synthase and postischemic liver injury. Biochem Biophys Res Commun. 2000;276(3):851-4.

192. Hines IN, Harada H, Bharwani S, Pavlick KP, Hoffman JM, Grisham MB. Enhanced post-ischemic liver injury in iNOS-deficient mice: a cautionary note. Biochem Biophys Res Commun. 2001;284(4):972-6.

193. Lang JD, Jr., Teng X, Chumley P, Crawford JH, Isbell TS, Chacko BK, et al. Inhaled NO accelerates restoration of liver function in adults following orthotopic liver transplantation. J Clin Invest. 2007;117(9):2583-91.

194. Lang JD, Jr., Smith AB, Brandon A, Bradley KM, Liu Y, Li W, et al. A randomized clinical trial testing the anti-inflammatory effects of preemptive inhaled nitric oxide in human liver transplantation. PLoS One. 2014;9(2):e86053.

195. Abu-Amara M, Yang SY, Quaglia A, Rowley P, de Mel A, Tapuria N, et al. Nitric oxide is an essential mediator of the protective effects of remote ischaemic preconditioning in a mouse model of liver ischaemia/reperfusion injury. Clin Sci (Lond). 2011;121(6):257-66.

196. Shiva S, Gladwin MT. Nitrite mediates cytoprotection after ischemia/reperfusion by modulating mitochondrial function. Basic Res Cardiol. 2009;104(2):113-9.

197. Cooper CE, Brown GC. The inhibition of mitochondrial cytochrome oxidase by the gases carbon monoxide, nitric oxide, hydrogen cyanide and hydrogen sulfide: chemical mechanism and physiological significance. J Bioenerg Biomembr. 2008;40(5):533-9.

198. Cleeter MW, Cooper JM, Darley-Usmar VM, Moncada S, Schapira AH. Reversible inhibition of cytochrome $\mathrm{c}$ oxidase, the terminal enzyme of the mitochondrial respiratory chain, by nitric oxide. Implications for neurodegenerative diseases. FEBS Lett. 1994;345(1):50-4.

199. Schweizer M, Richter C. Nitric oxide potently and reversibly deenergizes mitochondria at low oxygen tension. Biochem Biophys Res Commun. 1994;204(1):169-75.

200. Brown GC, Cooper CE. Nanomolar concentrations of nitric oxide reversibly inhibit synaptosomal respiration by competing with oxygen at cytochrome oxidase. FEBS Lett. 1994;356(2-3):295-8.

201. Murry CE, Jennings RB, Reimer KA. Preconditioning with ischemia: a delay of lethal cell injury in ischemic myocardium. Circulation. 1986;74(5):1124-36.

202. Kume M, Yamamoto Y, Saad S, Gomi T, Kimoto S, Shimabukuro T, et al. Ischemic preconditioning of the liver in rats: implications of heat shock protein induction to increase tolerance of ischemia-reperfusion injury. J Lab Clin Med. 1996;128(3):251-8.

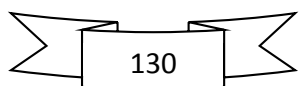


203. Saito T, Ishii S, Abe T, Tsuchiya T, Kanno H, Miyazawa M, et al. Effect of preconditioning in the liver against ischemia/reperfusion injury, protection of sinusoidal cells and alterations of gene transcription. Transplant Proc. 2001;33(1-2):849.

204. Yamada F, Abe T, Saito T, Tsuciya T, Ishii S, Gotoh M. Ischemic preconditioning enhances regenerative capacity of hepatocytes after prolonged ischemia. Transplant Proc. 2001;33(1-2):956.

205. Ishii S, Abe $T$, Saito $T$, Tsuchiya $T$, Kanno $H$, Miyazawa $M$, et al. Effects of preconditioning on ischemia/reperfusion injury of hepatocytes determined by immediate early gene transcription. J Hepatobiliary Pancreat Surg. 2001;8(5):461-8.

206. Tsuyama H, Shimizu K, Yoshimoto K, Nezuka H, Ito H, Yamamoto S, et al. Protective effect of ischemic preconditioning on hepatic ischemia-reperfusion injury in mice. Transplant Proc. 2000;32(7):2310-3.

207. Teoh N, Field J, Farrell G. Interleukin-6 is a key mediator of the hepatoprotective and pro-proliferative effects of ischaemic preconditioning in mice. $J$ Hepatol. 2006;45(1):20-7. Epub 2006 Mar 20.

208. Rudiger HA, Kang KJ, Sindram D, Riehle HM, Clavien PA. Comparison of ischemic preconditioning and intermittent and continuous inflow occlusion in the murine liver. Ann Surg. 2002;235(3):400-7.

209. Yadav SS, Sindram D, Perry DK, Clavien PA. Ischemic preconditioning protects the mouse liver by inhibition of apoptosis through a caspase-dependent pathway. Hepatology. 1999;30(5):1223-31.

210. Li JY, Gu X, Yin HZ, Zhou Y, Zhang WH, Qin YM. Protective effect of ischemic preconditioning on hepatic ischemia-reperfusion injury by advancing the expressive phase of survivin in rats. Hepatobiliary Pancreat Dis Int. 2008;7(6):615-20.

211. Teoh N, Dela Pena A, Farrell G. Hepatic ischemic preconditioning in mice is associated with activation of NF-kappaB, p38 kinase, and cell cycle entry. Hepatology. 2002;36(1):94-102.

212. Serafin A, Rosello-Catafau J, Prats N, Xaus C, Gelpi E, Peralta C. Ischemic preconditioning increases the tolerance of Fatty liver to hepatic ischemia-reperfusion injury in the rat. Am J Pathol. 2002;161(2):587-601.

213. Peralta C, Prats N, Xaus C, Gelpi E, Rosello-Catafau J. Protective effect of liver ischemic preconditioning on liver and lung injury induced by hepatic ischemiareperfusion in the rat. Hepatology. 1999;30(6):1481-9.

214. Schiesser M, Wittert A, Nieuwenhuijs VB, Morphett A, Padbury RT, Barritt GJ. Intermittent ischemia but not ischemic preconditioning is effective in restoring bile flow after ischemia reperfusion injury in the livers of aged rats. J Surg Res. 2009;152(1):618. doi: 10.1016/j.jss.2008.01.007. Epub Feb 1.

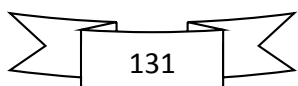


215. Park Y, Hirose R, Coatney JL, Ferrell L, Behrends M, Roberts JP, et al. Ischemiareperfusion injury is more severe in older versus young rat livers. J Surg Res. 2007;137(1):96-102. Epub 2006 Oct 24.

216. Sindram D, Rudiger HA, Upadhya AG, Strasberg SM, Clavien PA. Ischemic preconditioning protects against cold ischemic injury through an oxidative stress dependent mechanism. J Hepatol. 2002;36(1):78-84.

217. Schauer RJ, Gerbes AL, Vonier D, op den Winkel M, Fraunberger P, Bilzer M. Induction of cellular resistance against Kupffer cell-derived oxidant stress: a novel concept of hepatoprotection by ischemic preconditioning. Hepatology. 2003;37(2):28695 .

218. Arai M, Thurman RG, Lemasters JJ. Contribution of adenosine A(2) receptors and cyclic adenosine monophosphate to protective ischemic preconditioning of sinusoidal endothelial cells against Storage/Reperfusion injury in rat livers. Hepatology. 2000;32(2):297-302.

219. Peralta C, Hotter G, Closa D, Gelpi E, Bulbena O, Rosello-Catafau J. Protective effect of preconditioning on the injury associated to hepatic ischemia-reperfusion in the rat: role of nitric oxide and adenosine. Hepatology. 1997;25(4):934-7.

220. $\mathrm{Hu} \mathrm{GH}, \mathrm{Lu} \mathrm{XS}$. Effect of normothermic liver ischemic preconditioning on the expression of apoptosis-regulating genes $\mathrm{C}$-jun and $\mathrm{Bcl}-\mathrm{XL}$ in rats. World $\mathrm{J}$ Gastroenterol. 2005;11(17):2579-82.

221. Teoh N, Leclercq I, Pena AD, Farrell G. Low-dose TNF-alpha protects against hepatic ischemia-reperfusion injury in mice: implications for preconditioning. Hepatology. 2003;37(1):118-28.

222. Wang Y, Mathews WR, Guido DM, Farhood A, Jaeschke H. Inhibition of nitric oxide synthesis aggravates reperfusion injury after hepatic ischemia and endotoxemia. Shock. 1995;4(4):282-8.

223. Wang Y, Lawson JA, Jaeschke H. Differential effect of 2-aminoethyl-isothiourea, an inhibitor of the inducible nitric oxide synthase, on microvascular blood flow and organ injury in models of hepatic ischemia-reperfusion and endotoxemia. Shock. 1998;10(1):20-5.

224. Barrier A, Olaya N, Chiappini F, Roser F, Scatton O, Artus C, et al. Ischemic preconditioning modulates the expression of several genes, leading to the overproduction of IL-1Ra, iNOS, and Bcl-2 in a human model of liver ischemiareperfusion. FASEB J. 2005;19(12):1617-26.

225. Scatton O, Zalinski S, Jegou D, Compagnon P, Lesurtel M, Belghiti J, et al. Randomized clinical trial of ischaemic preconditioning in major liver resection with intermittent Pringle manoeuvre. Br J Surg. 2011;98(9):1236-43.

226. Clavien PA, Yadav S, Sindram D, Bentley RC. Protective effects of ischemic preconditioning for liver resection performed under inflow occlusion in humans. Ann Surg. 2000;232(2):155-62.

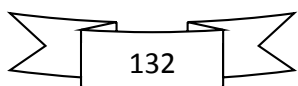


227. Clavien PA, Selzner M, Rudiger HA, Graf R, Kadry Z, Rousson V, et al. A prospective randomized study in 100 consecutive patients undergoing major liver resection with versus without ischemic preconditioning. Ann Surg. 2003;238(6):843-50; discussion $51-2$.

228. Chouker A, Martignoni A, Schauer R, Dugas M, Rau HG, Jauch KW, et al. Beneficial effects of ischemic preconditioning in patients undergoing hepatectomy: the role of neutrophils. Arch Surg. 2005;140(2):129-36.

229. Heizmann O, Loehe F, Volk A, Schauer RJ. Ischemic preconditioning improves postoperative outcome after liver resections: a randomized controlled study. Eur J Med Res. 2008;13(2):79-86.

230. Nuzzo G, Giuliante F, Vellone M, De Cosmo G, Ardito F, Murazio M, et al. Pedicle clamping with ischemic preconditioning in liver resection. Liver Transpl. 2004;10(2 Suppl 1):S53-7.

231. Petrowsky H, McCormack L, Trujillo M, Selzner M, Jochum W, Clavien PA. A prospective, randomized, controlled trial comparing intermittent portal triad clamping versus ischemic preconditioning with continuous clamping for major liver resection. Ann Surg. 2006;244(6):921-8; discussion 8-30.

232. Hahn O, Blazovics A, Vali L, Kupcsulik PK. The effect of ischemic preconditioning on redox status during liver resections--randomized controlled trial. J Surg Oncol. 2011;104(6):647-53.

233. Li SQ, Liang LJ, Huang JF, Li Z. Ischemic preconditioning protects liver from hepatectomy under hepatic inflow occlusion for hepatocellular carcinoma patients with cirrhosis. World J Gastroenterol. 2004;10(17):2580-4.

234. Azoulay D, Lucidi V, Andreani P, Maggi U, Sebagh M, Ichai P, et al. Ischemic preconditioning for major liver resection under vascular exclusion of the liver preserving the caval flow: a randomized prospective study. J Am Coll Surg. 2006;202(2):203-11.

235. Smyrniotis V, Theodoraki K, Arkadopoulos N, Fragulidis G, Condi-Pafiti A, PlemenouFragou $\mathrm{M}$, et al. Ischemic preconditioning versus intermittent vascular occlusion in liver resections performed under selective vascular exclusion: a prospective randomized study. Am J Surg. 2006;192(5):669-74.

236. Arkadopoulos N, Kostopanagiotou G, Theodoraki K, Farantos C, Theodosopoulos T, Stafyla $\mathrm{V}$, et al. Ischemic preconditioning confers antiapoptotic protection during major hepatectomies performed under combined inflow and outflow exclusion of the liver. A randomized clinical trial. World J Surg. 2009;33(9):1909-15.

237. Jeon J, Watkins A, Wagener G, Samstein B, Guarrera J, Goldstein M, et al. Complex hepatectomy under total vascular exclusion of the liver: impact of ischemic preconditioning on clinical outcomes. World J Surg. 2013;37(4):838-46.

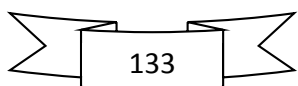


238. Gurusamy KS, Kumar Y, Pamecha V, Sharma D, Davidson BR. Ischaemic preconditioning for elective liver resections performed under vascular occlusion. Cochrane Database Syst Rev. 2009(1):CD007629.

239. Tapuria N, Kumar Y, Habib MM, Abu Amara M, Seifalian AM, Davidson BR. Remote ischemic preconditioning: a novel protective method from ischemia reperfusion injury-a review. J Surg Res. 2008;150(2):304-30.

240. Przyklenk K, Bauer B, Ovize M, Kloner RA, Whittaker P. Regional ischemic 'preconditioning' protects remote virgin myocardium from subsequent sustained coronary occlusion. Circulation. 1993;87(3):893-9.

241. Kanoria S, Jalan R, Davies NA, Seifalian AM, Williams R, Davidson BR. Remote ischaemic preconditioning of the hind limb reduces experimental liver warm ischaemiareperfusion injury. Br J Surg. 2006;93(6):762-8.

242. Lai IR, Chang KJ, Chen CF, Tsai HW. Transient limb ischemia induces remote preconditioning in liver among rats: the protective role of heme oxygenase-1. Transplantation. 2006;81(9):1311-7.

243. Gustafsson BI, Friman S, Wallin M, Heiman J, Delbro DS. Effect of remote preconditioning on mild or severe ischemia-reperfusion injury to rat liver. Transplant Proc. 2006;38(8):2708-9.

244. Tapuria N, Junnarkar SP, Dutt N, Abu-Amara M, Fuller B, Seifalian AM, et al. Effect of remote ischemic preconditioning on hepatic microcirculation and function in a rat model of hepatic ischemia reperfusion injury. HPB (Oxford). 2009;11(2):108-17.

245. Wang F, Birch SE, He R, Tawadros P, Szaszi K, Kapus A, et al. Remote ischemic preconditioning by hindlimb occlusion prevents liver ischemic/reperfusion injury: the role of High Mobility Group-Box 1. Ann Surg. 2010;251(2):292-9.

246. Abu-Amara M, Yang SY, Quaglia A, Rowley P, Tapuria N, Seifalian AM, et al. Effect of remote ischemic preconditioning on liver ischemia/reperfusion injury using a new mouse model. Liver Transpl. 2011;17(1):70-82.

247. Abu-Amara M, Yang SY, Quaglia A, Rowley P, Fuller B, Seifalian A, et al. Role of endothelial nitric oxide synthase in remote ischemic preconditioning of the mouse liver. Liver Transpl. 2011;17(5):610-9.

248. Abu-Amara M, Yang SY, Quaglia A, Rowley P, Tapuria N, Fuller B, et al. The hepatic soluble guanylyl cyclase-cyclic guanosine monophosphate pathway mediates the protection of remote ischemic preconditioning on the microcirculation in liver ischemiareperfusion injury. Transplantation. 2012;93(9):880-6.

249. Tapuria N, Junnarkar S, Abu-Amara M, Fuller B, Seifalian AM, Davidson BR. Modulation of microcirculatory changes in the late phase of hepatic ischaemiareperfusion injury by remote ischaemic preconditioning. HPB (Oxford). 2012;14(2):8797.

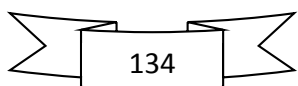


250. Wang M, Shen J, Feng B, Gui L, Chen Q, Zhang B, et al. Remote ischemic preconditioning promotes early liver cell proliferation in a rat model of small-for-size liver transplantation. J Surg Res. 2013;179(1):e245-53.

251. Kanoria S, Mehta NN, Seifalian A, Sharma D, Davidson B. Effect of remote ischemic preconditioning on liver injury in patients undergoing hepatectomy for colorectal metastasis: a single center randomised controlled trial. Br J Surg. 2010;97(S6):1.

252. Bito L, Davson H, Levin E, Murray M, Snider N. The concentrations of free amino acids and other electrolytes in cerebrospinal fluid, in vivo dialysate of brain, and blood plasma of the dog. J Neurochem. 1966;13(11):1057-67.

253. Ungerstedt U, Pycock C. Functional correlates of dopamine neurotransmission. Bull Schweiz Akad Med Wiss. 1974;30(1-3):44-55.

254. Heinemann L. Continuous glucose monitoring by means of the microdialysis technique: underlying fundamental aspects. Diabetes technology \& therapeutics. 2003;5(4):545-61.

255. Anderson $\mathrm{C}$, Andersson T, Wardell K. Changes in skin circulation after insertion of a microdialysis probe visualized by laser Doppler perfusion imaging. The Journal of investigative dermatology. 1994;102(5):807-11.

256. Bolinder J, Hagstrom E, Ungerstedt U, Arner P. Microdialysis of subcutaneous adipose tissue in vivo for continuous glucose monitoring in man. Scand J Clin Lab Invest. 1989;49(5):465-74.

257. Langberg H, Skovgaard D, Karamouzis M, Bulow J, Kjaer M. Metabolism and inflammatory mediators in the peritendinous space measured by microdialysis during intermittent isometric exercise in humans. J Physiol. 1999;515 ( Pt 3):919-27.

258. Nowak G, Ungerstedt J, Wernerman J, Ungerstedt U, Ericzon BG. Clinical experience in continuous graft monitoring with microdialysis early after liver transplantation. $\mathrm{Br} \mathrm{J}$ Surg. 2002;89(9):1169-75.

259. Hillered L, Persson L, Ponten U, Ungerstedt U. Neurometabolic monitoring of the ischaemic human brain using microdialysis. Acta neurochirurgica. 1990;102(3-4):91-7.

260. Meyerson BA, Linderoth B, Karlsson H, Ungerstedt U. Microdialysis in the human brain: extracellular measurements in the thalamus of parkinsonian patients. Life Sci. 1990;46(4):301-8.

261. Hrydziuszko O, Silva MA, Perera MT, Richards DA, Murphy N, Mirza D, et al. Application of metabolomics to investigate the process of human orthotopic liver transplantation: a proof-of-principle study. Omics. 2010;14(2):143-50.

262. Isaksson B, D'Souza M A, Jersenius U, Ungerstedt J, Lundell L, Permert J, et al. Continuous assessment of intrahepatic metabolism by microdialysis during and after portal triad clamping. J Surg Res. 2011;169(2):214-9.

263. Winbladh A, Sandstrom P, Olsson H, Svanvik J, Gullstrand P. Segmental ischemia of the liver - microdialysis in a novel porcine model. Eur Surg Res. 2009;43(3):276-85.

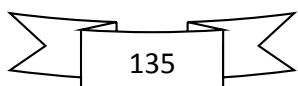


264. Spiegel HU, Bahde R. Experimental models of temporary normothermic liver ischemia. J Invest Surg. 2006;19(2):113-23.

265. Suzuki S, Toledo-Pereyra LH, Rodriguez FJ, Cejalvo D. Neutrophil infiltration as an important factor in liver ischemia and reperfusion injury. Modulating effects of FK506 and cyclosporine. Transplantation. 1993;55(6):1265-72.

266. Peralta C, Rull R, Rimola A, Deulofeu R, Rosello-Catafau J, Gelpi E, et al. Endogenous nitric oxide and exogenous nitric oxide supplementation in hepatic ischemia-reperfusion injury in the rat. Transplantation. 2001;71(4):529-36.

267. Calabrese F, Valente M, Pettenazzo E, Ferraresso M, Burra P, Cadrobbi R, et al. The protective effects of L-arginine after liver ischaemia/reperfusion injury in a pig model. $\mathrm{J}$ Pathol. 1997;183(4):477-85.

268. Winbladh A, Bjornsson B, Trulsson L, Bojmar L, Sundqvist T, Gullstrand P, et al. Nacetyl cysteine improves glycogenesis after segmental liver ischemia and reperfusion injury in pigs. Scand J Gastroenterol. 2012;47(2):225-36.

269. Zhang Z, Naughton DP, Blake DR, Benjamin N, Stevens CR, Winyard PG, et al. Human xanthine oxidase converts nitrite ions into nitric oxide (NO). Biochem Soc Trans. 1997;25(3):524S.

270. Cosby K, Partovi KS, Crawford JH, Patel RP, Reiter CD, Martyr S, et al. Nitrite reduction to nitric oxide by deoxyhemoglobin vasodilates the human circulation. Nat Med. 2003;9(12):1498-505.

271. Duranski MR, Greer JJ, Dejam A, Jaganmohan S, Hogg N, Langston W, et al. Cytoprotective effects of nitrite during in vivo ischemia-reperfusion of the heart and liver. J Clin Invest. 2005;115(5):1232-40.

272. Shiva S, Sack MN, Greer JJ, Duranski M, Ringwood LA, Burwell L, et al. Nitrite augments tolerance to ischemia/reperfusion injury via the modulation of mitochondrial electron transfer. J Exp Med. 2007;204(9):2089-102.

273. Park JW, Piknova B, Huang PL, Noguchi CT, Schechter AN. Effect of blood nitrite and nitrate levels on murine platelet function. PLoS One. 2013;8(2):e55699.

274. Govoni M, Tocchetti P, Lundberg JO. Metabolism and pathways for denitration of organic nitrates in the human liver. J Pharmacol Exp Ther. 2013;346(1):96-104.

275. Wang WD, Liang LJ, Huang XQ, Yin XY. Low central venous pressure reduces blood loss in hepatectomy. World J Gastroenterol. 2006;12(6):935-9.

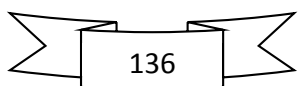




\section{Papers}

The articles associated with this thesis have been removed for copyright reasons. For more details about these see:

http://urn.kb.se/resolve?urn=urn:nbn:se:liu:diva-110318 